CERN-PH-TH/2011-122

\title{
The Other Natural Two Higgs Doublet Model
}

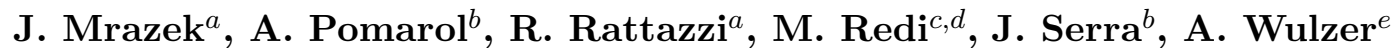 \\ ${ }^{a}$ Institut de Théorie des Phénomènes Physiques, EPFL, CH-1015 Lausanne, Switzerland \\ ${ }^{b}$ Departament de Física, Universitat Autònoma de Barcelona, 08193 Bellaterra (Barcelona), Spain \\ ${ }^{c}$ CERN, Theory Division, CH-1211 Geneva 23, Switzerland \\ ${ }^{d}$ INFN, 50019 Sesto F., Firenze, Italy \\ ${ }^{e}$ ETH Zurich, 8093 Zurich, Switzerland
}

\begin{abstract}
We characterize models where electroweak symmetry breaking is driven by two light Higgs doublets arising as pseudo-Nambu-Goldstone bosons of new dynamics above the weak scale. They represent the simplest natural two Higgs doublet alternative to supersymmetry. We construct their low-energy effective Lagrangian making only few specific assumptions about the strong sector. These concern their global symmetries, their patterns of spontaneous breaking and the sources of explicit breaking. In particular we assume that all the explicit breaking is associated with the couplings of the strong sector to the Standard Model fields, that is gauge and (proto)-Yukawa interactions. Under those assumptions the scalar potential is determined at lowest order by very few free parameters associated to the top sector. Another crucial property of our scenarios is the presence of a discrete symmetry, in addition to custodial $\mathrm{SO}(4)$, that controls the $T$-parameter. That can either be simple $C P$ or a $Z_{2}$ that distinguishes the two Higgs doublets. Among various possibilities we study in detail models based on $\mathrm{SO}(6) / \mathrm{SO}(4) \times$ $\mathrm{SO}(2)$, focussing on their predictions for the structure of the scalar spectrum and the deviations of their couplings from those of a generic renormalizable two Higgs doublet model.
\end{abstract}




\section{Contents}

1 Introduction 2

2 Two Composite Higgs Doublets as PNGBs 4

2.1 General Structure . . . . . . . . . . . . . . . . . . . . . . . . 4

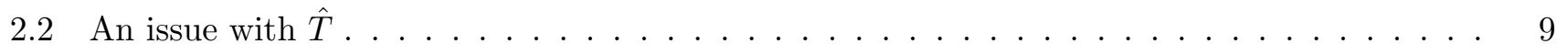

2.3 The Structure of Flavor . . . . . . . . . . . . . . . . . . . . . 11

2.4 Electroweak Precision Observables . . . . . . . . . . . . . . . . 16

3 Explicit Models $\quad 18$

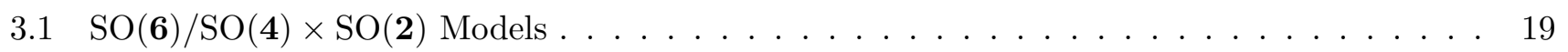

3.1 .1 Higgs Potential . . . . . . . . . . . . . . . . . . . 20

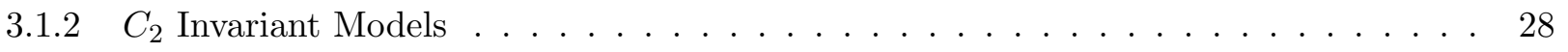

$3.1 .3 \quad C_{1} P$ Invariant Models $\ldots \ldots \ldots \ldots \ldots \ldots \ldots$

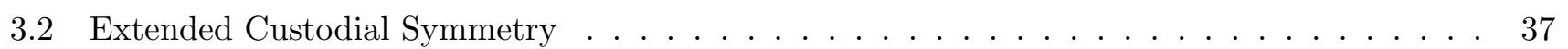

4 Phenomenology of Composite 2HDM 39

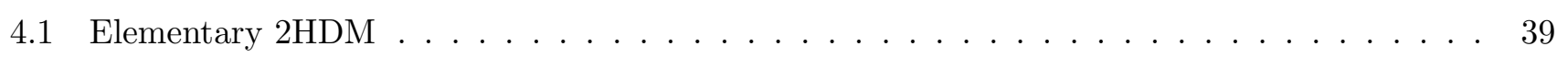

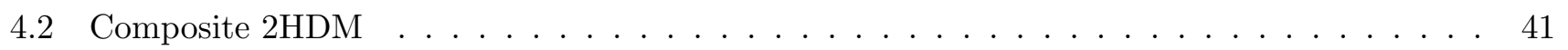

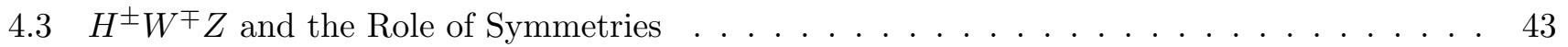

4.4 Phenomenology of $\mathrm{SO}(6) / \mathrm{SO}(4) \times \mathrm{SO}(2)$ Models $\ldots \ldots \ldots \ldots$. . . . . . . . . . . . . . . . . . . . .

4.4 Composite Inert Higgs . . . . . . . . . . . . . . . . . . 46

4.4 .2 Almost Inert Higgs . . . . . . . . . . . . . . . . . . . . . 47

$\begin{array}{llr}5 & \text { Summary } & 49\end{array}$

A Two Higgs SO(4)-invariant derivative interactions 53

\section{Introduction}

Uncovering the Higgs sector at the LHC is going to be a difficult but crucial task needed to understand the breaking of the electroweak symmetry. In the SM the Higgs sector consists of only one scalar $\mathrm{SU}(2)_{L^{-}}$ doublet, but models with a much more opulent Higgs structure have been extensively considered in the literature. Among them, two Higgs doublet models (2HDM) have attracted a lot of attention due to their rich phenomenology in electroweak and flavor physics.

The Higgs sector, however, consisting of scalars, is very sensitive to UV physics, giving rise to the well-known hierarchy problem. It is expected then that the new physics needed to solve this problem significantly affects the Higgs sector and its properties. This is exactly what happens in the most popular 
solution to the hierarchy problem, supersymmetry. There one learns that indeed the Higgs sector of the supersymmetric Standard Model is in fact quite restrictive, requiring two Higgs doublets with Yukawa and potential terms taking a very specific form. This shows that different Higgs scenarios, such as 2HDM, must be analyzed within frameworks that address at the same time the hierarchy problem.

The other natural alternative to supersymmetry, that also addresses the hierarchy problem, is to consider the Higgs bosons as composite states arising from a strong sector. The Higgses can be lighter than the strong scale, as favored by electroweak precision tests, if they are pseudo-Nambu-Goldstone bosons (PNGB) of an approximate symmetry $G$ spontaneously broken to $H$ [1, 2]. One very interesting aspect of these scenarios is that the low-energy dynamics is to a large extent determined by symmetry. For instance, the spectrum of light scalars is fixed by the coset $G / H$. Furthermore, the effective Lagrangian is constrained by the $G / H$ construction [3] and by the structure of the $G$-breaking couplings, that is by selection rules. In particular, the Higgs potential is fully determined up to a few $O(1)$ coefficients by the couplings between the strong sector and the Standard Model (SM). That provides information on the main phenomenological properties of these models without the necessity of a detailed knowledge of the strong sector.

In this paper we will explore composite PNGB Higgs models involving two Higgs doublets. Our interest is to show how these natural scenarios restrict generic 2HDM. The presence of two Higgs doublets, rather than just one, rises two main phenomenological challenges. The first concerns the breaking of the approximate custodial $\mathrm{SO}(3)_{c}$ symmetry by the vacuum structure of the model. That can lead to large contributions to the $T$-parameter even when the custodial symmetry is preserved by the strong sector. The second concerns Higgs-mediated Flavor Changing Neutral Currents (FCNC), which is a well known potential problem of theories with extended Higgs sectors. We will explain how these two problems can be overcome in a natural way by the use of discrete symmetries. These discrete symmetries restrict the form of the Higgs potential and of the Yukawa couplings, thus leading to interesting predictions. We are lead to considering two classes of models. In the first class, like in the inert Higgs model [4], the extra Higgs doublet will be odd under a certain parity, $C_{2}$. In the limit of exact $C_{2}$ the second Higgs does not couple linearly to the SM fields. In the second class, an approximate $C P$ symmetry will control the $T$-parameter and FCNC. The simplest models we could contruct in this second class, however, feature, somewhat unexpectedly, an accidental approximate $C_{2}$ parity, thus giving rise to "almost inert" Higgs scenarios.

The structure of the paper is as follows. In section 2 we describe the general structure of composite $2 \mathrm{HDM}$, pointing out how discrete symmetries can help to avoid constraints from the $T$-parameter and FCNC. In section 3 we present explicit composite $2 \mathrm{HDM}$ models. We mainly concentrate in $2 \mathrm{HDM}$ arising from the $\mathrm{SO}(6) / \mathrm{SO}(4) \times \mathrm{SO}(2)$ coset, although we will also briefly present models with extended custodial symmetry such as those based on the $\mathrm{Sp}(6) / \mathrm{SU}(2) \times \mathrm{Sp}(4)$ coset. In section 4 we give the main phenomenological implications of composite 2HDM. We focus first on model-independent features and later concentrate on the phenomenology of two particular examples, the composite inert Higgs and the almost 


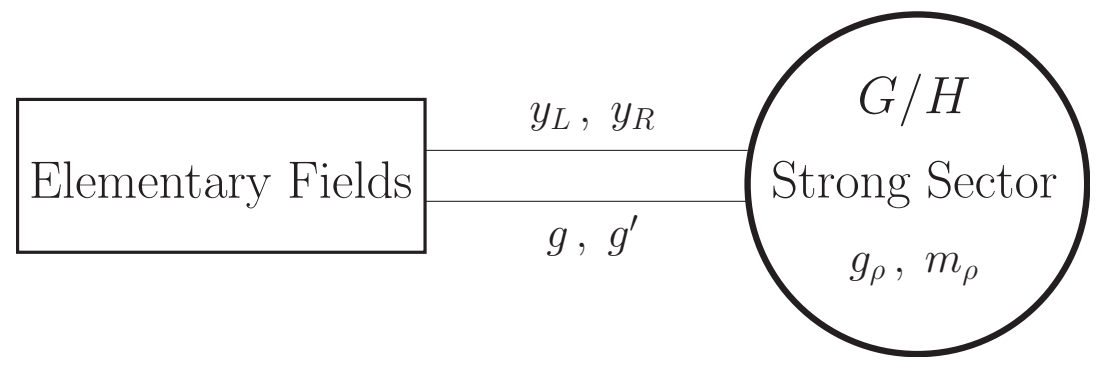

Figure 1: Pictorial representation of our scenario.

composite inert Higgs. The last section is devoted to conclusions.

\section{Two Composite Higgs Doublets as PNGBs}

\subsection{General Structure}

The basic structure of our composite-Higgs scenario is as follows. As depicted in figure 1, there exists a new sector, that we denote as "strong", or "strongly-interacting" sector, which is endowed with a global group $G$ of symmetry, spontaneously broken to $H \subset G$. As such, the strong sector delivers a set of massless Nambu-Goldstone bosons (NGB). The only constraints on the choice of the $G / H$ coset that characterizes the strong sector are of phenomenological nature and they are rather mild, a priori. The main requirement, needed to avoid generic large contributions to the $T$-parameter, is that the unbroken group must contain a "custodial" $\mathrm{SO}(4) \cong \mathrm{SU}(2) \times \mathrm{SU}(2)$ symmetry, $H \supset \mathrm{SO}(4)$, and at least one Higgs 4-plet (i.e., a 4 of $\mathrm{SO}(4))$ must be present. Compatibly with these basic requirements, several cosets exist. The smallest ones, chosen so that $H$ is a maximal subgroup of $G$, are present in table 1 . Other cosets, with non-maximal

\begin{tabular}{cccc}
\hline$G$ & $H$ & $N_{G}$ & NGBs rep. $[H]=$ rep. $[\mathrm{SU}(2) \times \mathrm{SU}(2)]$ \\
$\mathrm{SO}(5)$ & $\mathrm{SO}(4)$ & 4 & $\mathbf{4}=(\mathbf{2}, \mathbf{2})$ \\
$\mathrm{SO}(6)$ & $\mathrm{SO}(5)$ & 5 & $\mathbf{5}=(\mathbf{1}, \mathbf{1})+(\mathbf{2}, \mathbf{2})$ \\
$\mathrm{SO}(6)$ & $\mathrm{SO}(4) \times \mathrm{SO}(2)$ & 8 & $\mathbf{4}_{+\mathbf{2}}+\overline{\mathbf{4}}_{-\mathbf{2}}=2 \times(\mathbf{2}, \mathbf{2})$ \\
$\mathrm{SO}(7)$ & $\mathrm{SO}(6)$ & 6 & $\mathbf{6}=2 \times(\mathbf{1}, \mathbf{1})+(\mathbf{2}, \mathbf{2})$ \\
$\mathrm{SO}(7)$ & $\mathrm{G}_{2}$ & 7 & $\mathbf{7}=(\mathbf{1}, \mathbf{3})+(\mathbf{2}, \mathbf{2})$ \\
$\mathrm{SO}(7)$ & $\mathrm{SO}(5) \times \mathrm{SO}(2)$ & 10 & $\mathbf{1 0}_{\mathbf{0}}=(\mathbf{3}, \mathbf{1})+(\mathbf{1}, \mathbf{3})+(\mathbf{2}, \mathbf{2})$ \\
$\mathrm{SO}(7)$ & {$[\mathrm{SO}(3)]^{3}$} & 12 & $(\mathbf{2}, \mathbf{2}, \mathbf{3})=3 \times(\mathbf{2}, \mathbf{2})$ \\
$\mathrm{Sp}(6)$ & $\mathrm{Sp}(4) \times \mathrm{SU}(2)$ & 8 & $(\mathbf{4}, \mathbf{2})=2 \times(\mathbf{2}, \mathbf{2}),(\mathbf{2}, \mathbf{2})+2 \times(\mathbf{2}, \mathbf{1})$ \\
$\mathrm{SU}(5)$ & $\mathrm{SU}(4) \times \mathrm{U}(1)$ & 8 & $\mathbf{4}_{-5}+\overline{\mathbf{4}}_{+\mathbf{5}}=2 \times(\mathbf{2}, \mathbf{2})$ \\
$\mathrm{SU}(5)$ & $\mathrm{SO}(5)$ & 14 & $\mathbf{1 4}=(\mathbf{3}, \mathbf{3})+(\mathbf{2}, \mathbf{2})+(\mathbf{1}, \mathbf{1})$ \\
\hline
\end{tabular}

Table 1: Cosets $G / H$ from simple Lie groups, with $H$ maximal subgroup of $G$. For each coset, its dimension $N_{G}$ and the NGBs representation under $H$ and $\mathrm{SO}(4) \simeq \mathrm{SU}(2)_{L} \times \mathrm{SU}(2)_{R}$ are reported. For $\mathrm{Sp}(6) / \mathrm{SU}(2) \times \mathrm{Sp}(4)$, two embeddings are possible, we will be interested only in the first one, which leads to two Higgs 4-plets.

subgroups, can be obtained from table 1 in a stepwise fashion $G \rightarrow H \rightarrow H^{\prime}$ etc.. The coset $\mathrm{SO}(6) / \mathrm{SO}(4)$, for instance, arises from the breaking $\mathrm{SO}(6) \rightarrow \mathrm{SO}(5) \rightarrow \mathrm{SO}(4)$. Besides two $(2,2)$ Higgs 4-plets, this coset 
contains an extra scalar singlet $(\mathbf{1}, \mathbf{1})$. The cosets that only contain two Higgs doublets, and therefore give rise to a composite Two Higgs Doublet Model $(2 \mathrm{HDM})$, are $\mathrm{SO}(6) / \mathrm{SO}(4) \times \mathrm{SO}(2), \mathrm{Sp}(6) / \mathrm{SU}(2) \times \mathrm{Sp}(4)$, and $\mathrm{SU}(5) / \mathrm{SU}(4) \times \mathrm{U}(1)$. In the following, when discussing explicit realizations of the composite $2 \mathrm{HDM}$ scenario, we will mainly consider the $\mathrm{SO}(6) / \mathrm{SO}(4) \times \mathrm{SO}(2)$ coset, but the $\mathrm{Sp}(6) / \mathrm{SU}(2) \times \mathrm{Sp}(4)$ one will also find an interesting application, in section 3.2 , as an example of models with an extended custodial symmetry group.

Apart from the choice of the $G / H$ symmetry breaking pattern, very mild assumptions will be made on the nature of the strong sector and on its microscopic origin. In the spirit of [5], we assume its dynamics to be controlled by the smallest possible set of parameters: a coupling $g_{\rho} \leq 4 \pi$ that controls the interactions of the strong sector's resonances and the typical size $m_{\rho}$ of their masses. One possible implementation of this scenario could be provided by strongly-interacting confining "QCD-like" gauge theories in the large- $N$ expansion. At large- $N$, the size of all the couplings among mesonic resonances is fixed by

$$
g_{\rho} \simeq \frac{4 \pi}{\sqrt{N}},
$$

while the mass $m_{\rho} \sim \Lambda_{S}$ is set by the confinement scale and does not depend on $N$. Other realizations of our strong sector, which are definitely easier to construct and to deal with, are the holographic fivedimensional models, discussed at length in the literature for the case of the "minimal" $\mathrm{SO}(5) / \mathrm{SO}(4)$ coset [2, 6].

At energies below the resonance scale $m_{\rho}$, independently of their microscopic origin, the NGB composite Higgses are described by the non-linear $\sigma$-model associated to the $G / H$ coset. At the leading two-derivatives order, the sigma-model interactions are dictated by the dimension-full coupling $1 / f$ which, given our assumptions on the strong sector, has to be identified with $g_{\rho} / m_{\rho}$, leading to the relation $m_{\rho} \simeq g_{\rho} f$. Notice that it is only if the NGB form an irreducible representation of $H$ that their two-derivative interactions are completely fixed, and therefore predicted, in terms of a unique parameter $f$. This is the case for all the cosets in table 1, while for instance in $\mathrm{SO}(6) / \mathrm{SO}(4)$ the most general two derivative Lagrangian is described by four parameters associated to the four quadratic invariants which can be built out of two 4-plets and one singlet 2 ,

There are strong phenomenological hints, some of which will be summarized in the following, that the observed quarks and leptons (with the possible remarkable exception of the right-handed top quark $t_{R}$ ) and the transverse polarizations of the EW gauge bosons are not composite objects of some stronglyinteracting dynamics, or at least that they are not entirely composite. We therefore need to introduce these particles as "elementary fields", external to the strong sector, and make them communicate with the

\footnotetext{
${ }^{1}$ Notice that the "universality" of the coupling only holds in the mesonic sector, while resonances of different nature can interact with parametrically different couplings. For instance, for the glueballs in QCD, $g_{G} \simeq 4 \pi / N$. Thus if we needed to account for all classes of resonances we would not be able to depict the strong sector just in terms of a single coupling $g_{\rho}$. We shall assume that only mesons matter and work with a single coupling. Based on 5D examples, that is not an unreasonable assumption. Moreover phenomenological constraint prefer a large $g_{\rho}$, in which case all distinctions disappear.

${ }^{2}$ By performing field redefinitions one can however show that only three parameters are physically independent.
} 
latter by a set of couplings, to be defined later, $g, g^{\prime}, y_{L}, y_{R}$, as shown in figure 1 . We will generically denote these "elementary" couplings as $g_{S M}$. For what concerns the $\operatorname{SM~SU}(2)_{L} \times \mathrm{U}(1)_{Y}$ gauge fields, there is no ambiguity on how they should be coupled to the strong sector. The SM gauge group is identified as the appropriate subgroup of the global $\mathrm{SO}(4) \subset H$, and it is gauged with couplings $g$ and $g^{\prime}$. The standard gauging basically consists in writing down a linear coupling of the elementary gauge fields with the corresponding global currents of the strong sector.

The fermions also need to be coupled to the strong sector, with the aim of generating their masses, and this could be achieved in two ways. We could write bilinear terms, involving one left- and one right-handed fermion coupled to a bosonic strong sector operator with the quantum numbers of the Higgs. This is of course the standard mechanism for fermions mass generation in technicolor-like theories. Or, copying from what we just saw to happen for the gauge fields, we may adopt the "partial compositeness" paradigm [7, 2] and introduce linear terms, separately for the left- and right-handed components, which involve fermionic strong sector operators. In the present paper we will consider this second possibility, with $y_{L}$ and $y_{R}$ being the left- and right-handed fermion linear couplings, which we will denote as "proto-Yukawa" couplings. Schematically, the couplings of the elementary fields to the strong sector can be written as

$$
\mathcal{L}_{\text {mix }}=g_{S M} \cdot \Psi_{S M} \cdot \mathcal{O}
$$

where $\Psi_{S M}=\left(A_{\mu}, f\right)$ collectively denotes the SM gauge fields and fermions. Notice that, since the elementary states do not fill complete representation of $G, \mathcal{L}_{\text {mix }}$ unavoidably breaks the strong sector's global group. The Higgs therefore becomes a PNGB and is free to acquire a potential, as we will discuss below.

Because of these linear couplings, the SM fields have a degree of mixing

$$
\epsilon_{g} \equiv \frac{g}{g_{\rho}}, \quad \epsilon_{L, R} \equiv \frac{y_{L, R}}{g_{\rho}},
$$

with the strong sector's resonances. It is only when this mixing is not too large that the previouslymentioned phenomenological bounds can be accommodated and the model made realistic [5, 8]. This suggests that the coupling $g_{\rho}$ is better taken to be large, at least larger than the elementary couplings $g_{S M}$ 3. As in [5], we then restrict our parameter space to the region

$$
g_{S M} \leq g_{\rho} \leq 4 \pi
$$

where the limit of total compositeness $g_{S M} \simeq g_{\rho}$ could be considered for the $t_{R}\left(y_{R} \simeq g_{\rho}\right)$, given that phenomenological constraints on the $t_{R}$ compositeness are practically absent. Instead of taking $y_{R} \simeq g_{\rho}$, a more direct way to achieve total $t_{R}$ compositeness is not to introduce the elementary $t_{R}$ field to start with, and assume that a massless resonance with the quantum numbers of the $t_{R}$ emerges from the strong sector.

\footnotetext{
${ }^{3}$ As a matter of fact $g_{\rho}<g_{S M}$ would not even be a radiatively stable choice.
} 

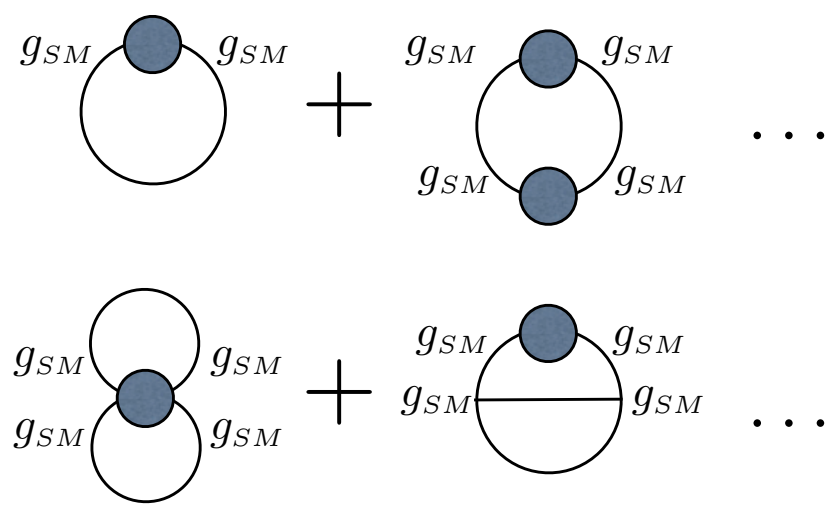

Figure 2: Power counting for the Higgs potential.

Due to the couplings in eq. (2) to the SM fermions, and in particular to the quarks, the strong sector must be charged under the full SM group, including the color $\mathrm{SU}(3)_{c}$. On top of the $G / H$ cosets discussed until now, and listed in table 1, the strong sector must therefore also enjoy an unbroken $\mathrm{SU}(3)_{c}$ global group, weakly gauged with coupling $g_{\text {strong }}$ by elementary gluon fields. This gluon gauge coupling should also appear in eq. (2), but it will be ignored since it does not play any role in what follows. Another unbroken symmetry of the strong sector that we have not mentioned is the strong sector matter charge $\mathrm{U}(1)_{X}$, which is needed to assign the correct hypercharge to the fermionic operators. The hypercharge is identified as $Y=T_{R}^{3}+X$, in terms of the third $\mathrm{SU}(2)_{R}$ generator $T_{R}^{3}$.

\section{The Structure of the Potential}

Let us briefly recall, for future use, the general structure of the effective potential of our PNGB Higgs.

In general, given a strong sector, one could imagine breaking its global symmetry $G$ either by adding new weak interactions among the composites or by their direct (weak) coupling to external elementary fields. For instance, in QCD the chiral symmetry is broken both by fermion masses, belonging to the first class of couplings, and by the coupling of quarks to the photon, which belongs to the second class. In our composite Higgs scenario, as described above, the second class of effects is always unavoidably present, while the first is not. It is thus not unreasonable, and also motivated by simplicity, to assume all the breaking of $G$ is due to the coupling to the SM fields in eq. (2). We will work under this assumption, bearing however in mind that by relaxing the latter the parameter space of PNGB Higgs models could be significantly enlarged.

Thanks to the above assumption, the potential only originates from insertions of the $g_{S M}$ couplings of eq. (2), and much can be said on its structure. First of all, its size can be estimated, as figure 2 shows, in an expansion in loops and in powers of the degree of the mixing $\epsilon=g_{S M} / g_{\rho}$. By noticing that each strong sector's $\langle\mathcal{O} \ldots \mathcal{O}\rangle$ correlator (represented as a circle in figure 2 is proportional to $1 / g_{\rho}^{2} \propto N$, the estimate 
reads

$$
V(\Pi)=\frac{m_{\rho}^{4}}{16 \pi^{2}}\left(\sum_{n=1}^{\infty} \epsilon^{2 n} F_{1 n}(\Pi / f)+\frac{g_{\rho}^{2}}{16 \pi^{2}} \sum_{n=1}^{\infty} \epsilon^{2 n} F_{2 n}(\Pi / f)+\text { higher loops }\right) .
$$

The Higgs bosons $\Pi$, because of their NGB nature, only appear in combination with the decay constant $f$, through the dimensionless functions $F_{i n}$. Second, but this will not be discussed in detail until section 3.1 the $G$ symmetry strongly constrains the possible contributions to the potential that arise at each given order in $g_{S M}$. This can be analyzed simply using spurion's power counting performed by assigning $G$ transformation properties to $g_{S M}$.

The generic properties of the EW vacuum and of the scalar spectrum are readily derived from the above equation. In the absence of tuning the generic minimum of the potential will be at $v=\langle\Pi\rangle \sim f$, and similarly the masses of the scalars scale as

$$
m_{\Pi}^{2} \sim \frac{g_{\rho}^{2}}{16 \pi^{2}} g_{S M}^{2} f^{2}\left(1+O\left(\epsilon^{2}\right)+\ldots\right)
$$

As quantitatively discussed in section 2.4 however, a certain amount of tuning in $v / f$ seems unavoidable for a realistic model. In order to perform such a tuning, one of the mass terms in the potential must be unnaturally reduced, while the quartic Higgs couplings must remain unaffected. This makes that the estimate of eq. (6) is typically violated, and along the "tuned direction" of the potential a lighter scalar $h$ emerges. Its mass is given by

$$
m_{h}^{2} \sim \frac{g_{\rho}^{2}}{16 \pi^{2}} g_{S M}^{2} v^{2}
$$

that is parametrically smaller than eq. (6). Up to effects $v^{2} / f^{2}$, the scalar $h$ behaves as the SM Higgs.

In the realistic cases the dominant source of the potential is given by the proto-Yukawas of the top quark, $y_{L}$ and $y_{R}$. These latter are indeed forced to be rather large because they have to reproduce the top Yukawa coupling $Y_{t} \simeq 1$, which is given by the relation

$$
Y_{t} \simeq \frac{y_{L} y_{R}}{g_{\rho}} .
$$

Because of eq. (4), one can deduce the lower bound

$$
\min \left(y_{L}, y_{R}\right) \gtrsim Y_{t} \quad \Rightarrow \quad m_{h}^{2} \gtrsim N_{c} \frac{g_{\rho}^{2} Y_{t}^{2}}{16 \pi^{2}} v^{2}
$$

where an $N_{c}=3$ factor representing the number of QCD colors has been added to the estimate of eq. (7). Notice that the lower bound above is only reached in the limit of total $t_{R}$ compositeness, $y_{L} \simeq Y_{t}$ and $y_{R} \simeq g_{\rho}$, but in other situations $h$ will be heavier. In realistic concrete cases (see sections 3.1 .2 and 3.1.3 the estimate of eq. (7) might however be violated by an extra accidental cancellation of the quartic coupling, and the Higgs could remain light. This notably occurs in the minimal $\mathrm{SO}(5) / \mathrm{SO}(4)$ composite Higgs model (MCHM) [2]. 


\subsection{An issue with $\hat{T}$}

After the general considerations of the previous section, let us now focus on the case of two composite Higgs doublets. As we will now discuss, an extra and very large contribution to the $\widehat{T}$ parameter, which is structurally absent in the single-Higgs case, potentially emerges. This is however very easily avoided.

In the SM with an elementary Higgs doublet, the accidental SO(4) symmetry of the Higgs sector ensures the survival, after EWSB, of an (approximate) custodial isospin $\mathrm{SO}(3)_{c}$. This symmetry is essential to successfully reproduce electroweak precision data, in particular the relation $\rho \equiv m_{W}^{2} / m_{Z}^{2} \cos ^{2} \theta_{W} \simeq 1$, or equivalently the bound on $\hat{T}$ (see [5] for the conventions). In the MCHM based on $\mathrm{SO}(5) / \mathrm{SO}(4)$, the $\mathrm{SO}(4)$ symmetry is a true symmetry of the strong dynamics, satisfied by all the non-linear $\sigma$-model interactions. The Higgs field, being a 4 of $\mathrm{SO}(4)$, determines a generic vacuum that again respect a residual custodial $\mathrm{SO}(3)_{c}$. An equivalent statement is that the gauged $\mathrm{SO}(4)_{g}$ and the residual $\mathrm{SO}(4)_{H}$ in the coset, when embedded in $\mathrm{SO}(5)$, have at least a common $\mathrm{SO}(3)$ subgroup. On the other hand, in non-minimal models with two Higgses in the 4 of $\mathrm{SO}(4)$ the generic residual symmetry of the vacuum will only be $\mathrm{SO}(2)_{c}$. The equivalent statement is that $\mathrm{SO}(4)_{g}$ and $\mathrm{SO}(4)_{H}$, when embedded in $\mathrm{SO}(6)$ generically have only an $\mathrm{SO}(2)$ common subgroup. Thus even though the nonlinear interactions satisfy $\mathrm{SO}(4)$, an unacceptable contribution to $\hat{T}$ will arise for a generic vacuum structure. To discuss this problem in more detail, it is useful to use two parametrizations of a 4 of $\mathrm{SO}(4)$, the one as a 4 -vector $\Phi=\left\{\phi_{i}\right\}, i=1, \ldots, 4$ and the one as a $2 \times 2$ matrix $\Phi \equiv \phi_{4}+i \phi_{k} \sigma_{k}(k=1,2,3)$ transforming as $\Phi \rightarrow L \Phi R^{\dagger}$ under $\mathrm{SO}(4) \cong \mathrm{SU}(2)_{L} \times \mathrm{SU}(2)_{R}$. We will use the same symbol $\Phi$ for both parametrizations, as it will be clear from the context which one we use 4

In a model with two Higgs fields $\Phi^{\hat{1}}$ and $\Phi^{\widehat{2}}$, up to $\mathrm{SU}(2)_{L} \times \mathrm{U}(1)_{Y}$ rotations, the generic charge preserving vacuum expectation value $(\mathrm{VEV})$ is $\Phi^{\widehat{1}}=\left(0,0,0, v_{4}^{\widehat{1}}\right), \Phi^{\widehat{2}}=\left(0,0, v_{3}^{\widehat{2}}, v_{4}^{\widehat{2}}\right)$. In Higgs doublet notation this corresponds to,

$$
H^{\widehat{1}}=\frac{1}{\sqrt{2}}\left(\begin{array}{c}
0 \\
v_{4}^{\hat{1}}
\end{array}\right) \quad H^{\widehat{2}}=\frac{1}{\sqrt{2}}\left(\begin{array}{c}
0 \\
v_{4}^{\widehat{2}}-i v_{3}^{\widehat{2}}
\end{array}\right),
$$

where, up to effects $v^{2} / f^{2}$, we have $v=\sqrt{\left(v_{4}^{\widehat{1}}\right)^{2}+\left(v_{4}^{\widehat{2}}\right)^{2}+\left(v_{3}^{\widehat{2}}\right)^{2}} \simeq 246 \mathrm{GeV}$.

It is easy to check that the operator

$$
\frac{c_{T}}{f^{2}}\left(\Phi^{\widehat{1}} \cdot \overleftrightarrow{D}_{\mu} \Phi^{\widehat{2}}\right)^{2}
$$

$\left(\Phi^{\widehat{1}} \cdot \overleftrightarrow{D}_{\mu} \Phi^{\widehat{2}}=\Phi^{\widehat{1}} \cdot\left(D_{\mu} \Phi^{\widehat{2}}\right)-\left(D_{\mu} \Phi^{\widehat{1}}\right) \cdot \Phi^{\widehat{2}}\right)$ which in general arises from the non-linearities of an $\mathrm{SO}(4)-$ symmetric $\sigma$-model, generates a contribution

$$
\widehat{T}=-8 c_{T} \frac{\left(v_{4}^{\widehat{1}}\right)^{2}\left(v_{3}^{\widehat{2}}\right)^{2}}{f^{2}\left[\left(v_{4}^{\widehat{\mathbf{1}}}\right)^{2}+\left(v_{4}^{\widehat{2}}\right)^{2}+\left(v_{3}^{\widehat{2}}\right)^{2}\right]},
$$

\footnotetext{
${ }^{4}$ In the matrix notation, the complex doublet is embedded as $\Phi=(\widetilde{H}, H)$ where $\widetilde{H}=i \sigma_{2} H^{*}$.
} 
proportional to the square of the order parameter $v_{4}^{\widehat{1}} v_{3}^{\widehat{2}}$ of $\mathrm{SO}(4) \rightarrow \mathrm{SO}(2)_{c}$ breaking. Notice that a contribution to $\widehat{T}$ is associated to $\operatorname{Im}\left(H^{\widehat{1} \dagger} H^{\widehat{2}}\right) \neq 0$. For $c_{T} \sim \mathcal{O}(1)$, as generically generated by $\sigma$-model interactions 5 and $v_{4}^{\widehat{1}} \sim v_{3}^{\widehat{2}} \sim v$, we would have $\widehat{T} \sim v^{2} / f^{2}$. That would be phenomenologically acceptable only at the price of significant tuning: $v^{2} / f^{2} \lesssim 0.002$.

Two discrete symmetries, $C_{1}$ and $C_{2}$, control the order parameter $v_{4}^{\widehat{1}} v_{3}^{\widehat{2}}$ and provide a useful organizing principle to describe vacuum dynamics:

- $C_{1}$ is the $Z_{2}$ subgroup of $\mathrm{SO}(4)$ acting on quadruplets as

$$
\left(\phi_{1}, \phi_{2}, \phi_{3}, \phi_{4}\right) \rightarrow\left(-\phi_{1}, \phi_{2},-\phi_{3}, \phi_{4}\right)
$$

or simply $H \rightarrow H^{*}$ in doublet notation. $C_{1}$, being a subgroup of $\mathrm{SO}(4)$, is respected by the strong sector in all models under consideration. It acts like charge conjugation on the Higgses, as we have seen, and on the $\mathrm{SU}(2)_{L} \times \mathrm{U}(1)_{Y}$ gauge bosons as well; it is thus broken when the SM fermions are taken into account. When fermions are included, $C_{1}$ may become an approximate symmetry only when combined with parity $P$, and that is just $C P$. Throughout the paper $C_{1} P$ is defined to act as standard $C P$ on the SM states. In particular it acts like $\psi \rightarrow \bar{\psi}$ without extra phases on the SM Weyl fermions.

- $C_{2}$ is a reflection in the $\left(\Phi^{\widehat{1}}, \Phi^{\widehat{2}}\right)$ plane, which without loss of generality we can choose to be $\Phi^{\widehat{1}} \rightarrow \Phi^{\widehat{1}}$, $\Phi^{\widehat{2}} \rightarrow-\Phi^{\hat{2}}$. This second symmetry is external to $\mathrm{SO}(4)$, it commutes with it and it may well be exact even when fermions are included. In $\mathrm{SO}(6) / \mathrm{SO}(4) \times \mathrm{SO}(2)$ and $\mathrm{SO}(6) / \mathrm{SO}(4)$ the role of $C_{2}$ can be played by the six-dimensional parity $P_{6}$. In that case those cosets would respectively be lifted to $\mathrm{O}(6) / \mathrm{SO}(4) \times \mathrm{O}(2)$ and $\mathrm{O}(6) / \mathrm{SO}(4) \times P_{2}$. In the case of $\mathrm{SU}(5) / \mathrm{SU}(4) \times \mathrm{U}(1)$ the role of $C_{2}$ can be played by charge conjugation in $\mathrm{SU}(5)$. It should be stressed that at the two derivative level the $\sigma$-model Lagrangian for $\mathrm{SO}(6) / \mathrm{SO}(4) \times \mathrm{SO}(2)$ and $\mathrm{SU}(5) / \mathrm{SU}(4) \times \mathrm{U}(1)$ are automatically endowed with $C_{2}$ : if the fundamental dynamics were to break $C_{2}$ that would only show up in the four- and higher-derivative Lagrangian, and in the interactions with the heavy composite states. On the other hand, the generic $\mathrm{SO}(6) / \mathrm{SO}(4)$ Lagrangian breaks $C_{2}$ already at the two derivative level. In that case $C_{2}$ can be imposed by suitably chosing the three independent coefficients that describe the two derivative $\sigma$-model action (remember $\mathrm{SO}(6) / \mathrm{SO}(4)$ is a reducible coset).

Combining $C_{1}, C_{2}$ and $P$ we have thus the following possibilities: ${ }^{6}$

1. $C_{1} P$ is an exact or approximate symmetry of the strong sector. If it is exact, it can also remain exact when only the third family fermions are included, but it will be definitely broken by the Yukawa couplings of the light families. Then the leading contribution to the Higgs potential will be $C_{1} P$ symmetric: $v_{4}^{\widehat{1}} v_{3}^{\widehat{2}}$ will only arise from small effects and will be well under control.

\footnotetext{
${ }^{5}$ In the particular case of the $\mathrm{SO}(6) / \mathrm{SO}(4) \times \mathrm{SO}(2)$ coset, one finds $c_{T}=-\frac{1}{4}$, that implies $\widehat{T}>0$.

${ }^{6}$ In the discussion that follows it is implicitly assumed that the vacuum respects the discrete symmetry under consideration. This typically happens in a region of paremeter space with non-zero measure.
} 
2. $C_{2}$ is an exact or approximate symmetry. If it is exact, $H^{\widehat{2}}$ acts like a composite inert Higgs [4], and the contribution to $\widehat{T}$ from the $\sigma$-model vanishes.

3. $C_{1} P \cdot C_{2}$ is an approximate symmetry only broken by the light family Yukawas, and it plays the role of $C P$. In this situation $v_{4}^{\widehat{1}} v_{3}^{\widehat{2}} \neq 0$, while $v_{4}^{\widehat{1}} v_{4}^{\widehat{2}}=0$ up to negligible effects, and the Higgs VEVs are anti-aligned. The custodial symmetry is maximally broken and the model is not viable. This is the situation encountered in the specific model discussed in ref. [9] .

4. No combination of $C_{1}, C_{2}$ and $P$ is even an approximate symmetry. In this situation $C P$ is violated at $O(1)$ by the top-Higgs sector, and also the custodial symmetry is broken at $O(1)$ by the VEV structure.

The above list exhausts all possibilities. We conclude that, in composite two Higgs doublet models, $\widehat{T}$ can be protected by either (approximate) $C P$ or (approximate or exact) $C_{2}$. Moreover it seems to us that the conditions for this protection, case 1 and 2, are rather mild and generic. In the potentially realistic models satisfying either condition 1 or 2 , the leading, and unavoidable, new physics contribution to $\widehat{T}$ typically comes from the top sector and its properties are the same as discussed in ref. [5]. After having discussed the structure of Yukawa couplings, we shall review the issue of electroweak precision parameters in section 2.4. There we will also make some novel remarks concerning the correlation between $\widehat{T}$ and the corrections to the $Z b \bar{b}$ vertex.

In the rest of the paper we shall mostly focus on the phenomenology of models of class 1 and 2 . There is however a third interesting possibility to control $\widehat{T}$, corresponding to a symmetry that allows to rotate $\Phi^{\widehat{2}}$ parallel to $\Phi^{\widehat{1}}$, or, which is the same, to a symmetry that constrains $c_{T}$ to vanish. Such a symmetry clearly cannot commute with $\mathrm{SO}(4)$ and should contain two $\mathrm{SU}(2)_{R}$ 's under which the two doublets transform independently: i.e. $\Phi^{\widehat{1}} \rightarrow L \Phi^{\hat{1}} R_{1}^{\dagger}$ and $\Phi^{\widehat{2}} \rightarrow L \Phi^{\hat{2}} R_{2}^{\dagger}$. The simplest coset where that occurs is $\mathrm{Sp}(6) / \mathrm{SU}(2) \times \mathrm{Sp}(4)$ in table 1. This third possibility is indeed the one which is accidentally realized in the weakly coupled case, such as in Supersymmetry. In a renormalizable theory, the kinetic terms are the only operators that give a mass to the vector bosons, and these are invariant under $\mathrm{SO}(8)$, explicitly broken to $\mathrm{SU}(2)_{L} \times \mathrm{Sp}(4)$ by the gauging of $\mathrm{SU}(2)_{L}$. $\mathrm{Sp}(4)$ contains two $\mathrm{SU}(2)_{R}$ under which each doublet transforms as above so that a custodial diagonal combination of the three $\mathrm{SU}(2)^{3}$ is preserved after both Higgses have taken arbitrary VEVs, implying $\widehat{T}=0$. Notice that for this to work only the kinetic terms must be invariant, not the entire Lagrangian. We shall further discuss the model building and phenomenology of this third class of models in section 3.2 .

\subsection{The Structure of Flavor}

One special feature of the renormalizable SM is that there exists only one matrix of flavor breaking (Yukawa) interactions associated to the fermions of any given charge. This ensures the absence at tree level of contributions to flavor changing neutral currents (FCNC) and is the zeroth order reason for the 
SM success in describing flavor breaking phenomena. This special feature, once called natural flavor conservation, and now dubbed Minimal Flavor Violation (MFV) [10, is "structurally" absent in virtually all extensions of the SM. That means that in the extensions of the SM to obtain the same simple structure additional symmetries or dynamical assumptions other than plain renormalizability 7 must be invoked.

In the 2HDM, focussing just on quarks, the most general Yukawa interaction is

$$
\bar{q}_{L}\left(Y_{1}^{u} \widetilde{H}_{\widehat{\mathbf{1}}}+Y_{2}^{u} \widetilde{H}_{\widehat{\mathbf{2}}}\right) u_{R}+\bar{q}_{L}\left(Y_{1}^{d} H_{\widehat{\mathbf{1}}}+Y_{2}^{d} H_{\widehat{\mathbf{z}}}\right) d_{R}+\text { h.c. },
$$

corresponding to four coupling matrices to generate the two mass matrices of the up and of the down quarks. The additional flavor breaking parameters give rise to dangerous flavor transitions via Higgs exchange, implying strong constraints on the parameters. A more plausible model can be obtained by restoring MFV, which can be done either by symmetry or by an ansatz. Using the same notation of the previous section, we can consider the Higgs parity symmetry $C_{2}$ under which $\left(H_{\widehat{1}}, H_{\widehat{2}}\right) \rightarrow\left(H_{\widehat{1}},-H_{\widehat{2}}\right)$ and all fermions are even, and the isospin parity $C_{I}$ under which $\left(u_{R}, d_{R}\right) \rightarrow\left(u_{R},-d_{R}\right)$ and all other fields are even. Then by imposing either $C_{2}$ or $C_{I} \cdot C_{2}$ the unwanted new sources of flavor violation are eliminated, and we go back to the minimal flavor violating structure of the SM. The two corresponding models are respectively known as type I and type II 2HDM. These and other options for the SM fermion parities, corresponding to different types of models present in the literature, are given in the table below:

\begin{tabular}{cccc}
\hline type & $u_{R}$ & $d_{R}$ & $e_{R}$ \\
\hline $\mathrm{I}$ & + & + & + \\
$\mathrm{II}$ & + & - & - \\
$\mathrm{X}$ & + & + & - \\
$\mathrm{Y}$ & + & - & + \\
\hline
\end{tabular}

The third possibility, known as type III, amounts to making the ansatz $Y_{1}^{u} \propto Y_{2}^{u}, Y_{1}^{d} \propto Y_{2}^{d}$, effectively enforcing MFV without any extra symmetry. This ansatz is consistent with selection rules from the flavor symmetry $\mathrm{SU}(3)_{q_{L}} \times \mathrm{SU}(3)_{u_{R}} \times \mathrm{SU}(3)_{d_{R}}$ and could in principle be motivated in a suitable model for the origin of flavor.

In composite Higgs models there are, a priori, extra sources of flavor violations in the Higgs sector [5, 9, 11]. For example, in the MCHM with only one Higgs doublet $H$ the most general structure of the Yukawa interactions (that is with zero derivatives) is 8

$$
\bar{q}_{L}\left(Y_{1}^{u} \tilde{H}+Y_{3}^{u} \tilde{H} H^{\dagger} H / f^{2}+\ldots\right) u_{R}+\bar{q}_{L}\left(Y_{1}^{d} H+Y_{3}^{d} H H^{\dagger} H / f^{2}+\ldots\right) d_{R}+\text { h.c. } .
$$

The matrices $Y_{3}^{u, d}$ generically give rise to flavor changing couplings to the neutral Higgs only suppressed, compared with the renormalizable ones in eq. (14), by $v^{2} / f^{2}$ which is typically not enough (see however

\footnotetext{
${ }^{7}$ Here, of course, we use the concept of renormalizability with its modern effective field theory meaning: we perform an inverse mass expansion and keep only relevant or marginal couplings.

${ }^{8}$ Other sources of flavor violation are associated with generalized kinetic terms with multiple Higgs insertions: these effects come at higher order in the Yukawa or proto-Yukawa couplings and are normally subdominant and not very problematic [11]. This is why we neglect them in our discussion.
} 

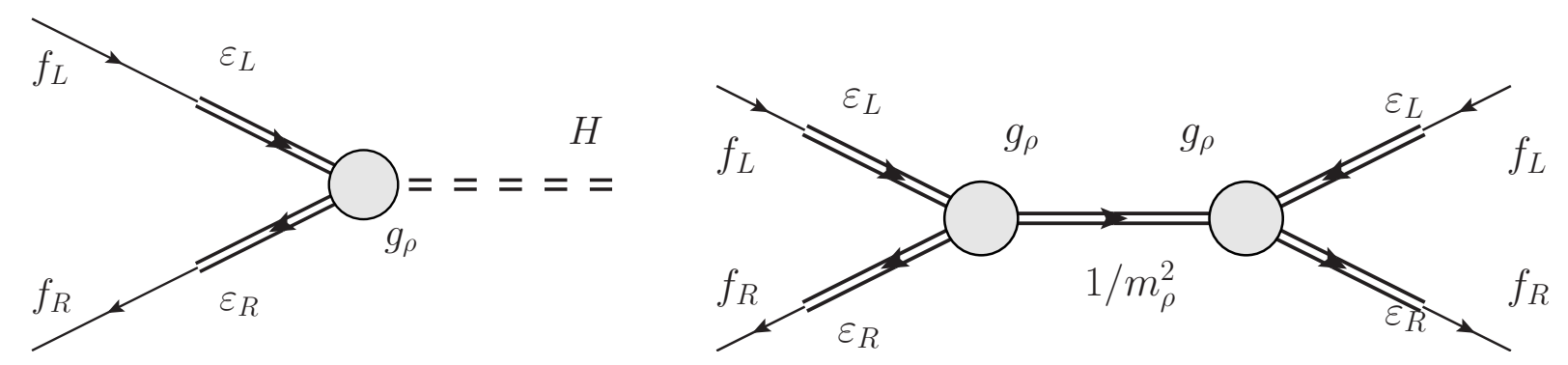

Figure 3: The contribution from the exchange of heavy modes to the Yukawas and to the FCNC operators.

the estimates that follow). The way out is again MFV, i.e. the conditions $Y_{1}^{u} \propto Y_{3}^{u} \propto \ldots$ and similarly for the downs. Interestingly, this can be automatically enforced in PNGB composite Higgs models where selection rules of the global group $G$ can imply, at lowest order in the proto-Yukawa couplings, a factorized flavor structure [1]

$$
\bar{q}_{L}\left(Y_{1}^{u} \tilde{H} F_{u}\left(H^{\dagger} H / f^{2}\right)\right) u_{R}+\bar{q}_{L}\left(Y_{1}^{d} H F_{d}\left(H^{\dagger} H / f^{2}\right)\right) d_{R}+h . c .
$$

This feature eliminates the leading contribution to Higgs-mediated FCNC.

Now, in the composite $2 \mathrm{HDM}$ the issues exemplified by eq. (14) and eq. (15) will both be present, but at the same time one will be able to rely, as explained above, on both, discrete symmetries or ansätze and on $G$ selection rules. Let us discuss in more detail how these mechanisms work and protect from Higgs-mediated flavor transitions. As previously explained, the SM fermions are coupled linearly to the strong sector through fermionic composite operators $\mathcal{O}_{f_{L}, f_{R}}$. The latter describe couplings at microscopic scales, where the breaking $G \rightarrow H$ can be neglected, and therefore correspond to some representations of $G$ that we denote, respectively, as $\mathbf{r}_{L}$ and $\mathbf{r}_{R}$. For one generation, eq. (2) can be rewritten more explicitly as

$$
\mathcal{L}_{\text {mix }}=\left(\bar{f}_{L}\right)_{\bar{\alpha}}\left(y_{L}{ }^{\bar{\alpha}}\right)^{I_{f_{L}}} \mathcal{O}_{I_{f_{L}}}+\left(\bar{f}_{R}\right)\left(y_{R}\right)^{I_{f_{R}}} \mathcal{O}_{I_{f_{R}}}+\text { h.c. }
$$

where the $I_{f_{L}}$ and $I_{f_{R}}$ indices of $y_{L, R}$ are in the conjugate representation of $\mathbf{r}_{L, R}$ while $\bar{\alpha}$ denotes the $\mathrm{SM} \mathrm{SU}(2)_{L}$-doublet index. As the notation suggests, in eq. (17) we have uplifted the $y_{L, R}$ couplings to representations (spurions) of the $G \times S U(2)_{W} \times U(1)_{Y}$. This will allow us to exploit fully the constraints from $G$-invariance.

Adding flavor to eq. (17), amounts to adding an index $i$ to $f_{L}, y_{L}, y_{R}, \mathcal{O}_{I_{f_{L}}}, \mathcal{O}_{I_{f_{R}}}$. Notice that in general there is no notion of orthogonality for the composite operators, meaning that the correlator $\left\langle\mathcal{O}_{I_{f_{L}}}^{i} \mathcal{O}_{I_{f_{L}}}^{j}\right\rangle$ is in general non zero for any $i, j$ pair (similarly for $\left.\mathcal{O}_{I_{f_{R}}}^{i}\right)$. Effective Yukawa couplings, in principle of the general form of eqs. (14) and (15), arise at low energy via the exchange of the heavy modes excited by $\mathcal{O}_{f_{L}, f_{R}}-$ see fig. 3. By applying power counting as depicted in the figure, we expect for the $Y_{1}^{i j}, Y_{2}^{i j}$ and 
$Y_{3}^{i j}$ in eqs. 14 15 the structure

$$
Y_{1,2,3}^{i j}=\frac{y_{L}^{i} y_{R}^{j}}{g_{\rho}} \times a_{1,2,3}^{i j}=g_{\rho} \epsilon_{L}^{i} \epsilon_{R}^{j} \times a_{1,2,3}^{i j}, \quad a_{1,2,3}^{i j} \sim O(1)
$$

with $a_{1}^{i j} \neq a_{2}^{i j} \neq a_{3}^{i j}$ in general. Notice that the size of the Yukawa of a given SM fermion is proportional to the degrees of mixing $\epsilon_{L}^{i}$ and $\epsilon_{R}^{i}$ of its chirality components to their composite counterparts. Assuming the strong sector does not have any flavor structure $\left(a_{1,2,3}^{i j} \sim O(1)\right)$ these mixings have to be hierarchical in order to reproduce the observed Yukawas. It is then straightforward to estimate the typical size of flavor violating transitions. The transitions mediated by heavy modes, as again depicted in figure 3 , give, for instance, $L R L R$ 4-fermi interactions

$$
\epsilon_{L}^{i} \epsilon_{R}^{j} \epsilon_{L}^{k} \epsilon_{R}^{\ell} \frac{g_{\rho}^{2}}{m_{\rho}^{2}}\left(\bar{f}_{L}^{i} f_{R}^{j} \bar{f}_{L}^{k} f_{R}^{\ell}\right)
$$

For instance for the $(\bar{d} s)^{2}, \Delta S=2$ transition, the coefficient is $\sim m_{d} m_{s} / v^{2} m_{\rho}^{2}$ which is small enough for the real part, while it puts some pressure on the parameters for $\epsilon_{K}[12$. Overall it is fair to say that this class of flavor violation can be under control with some, not totally implausible, mild tuning of parameters. On the other hand the FCNC mediated by the Higgses are usually larger. Generically, the lightest scalar $h$, that behaves as the MCHM Higgs, can mediate FCNC contributions from the flavor-changing couplings of eq. (15), while extra heavy scalars $S$ can mediate them from the couplings of eq. (14). These two types of FCNC contributions are respectively given by

$$
\epsilon_{L}^{i} \epsilon_{R}^{j} \epsilon_{L}^{k} \epsilon_{R}^{\ell} \frac{g_{\rho}^{2}}{m_{h}^{2}} \frac{v^{4}}{f^{4}}\left(\bar{f}_{L}^{i} f_{R}^{j} \bar{f}_{L}^{k} f_{R}^{\ell}\right), \quad \epsilon_{L}^{i} \epsilon_{R}^{j} \epsilon_{L}^{k} \epsilon_{R}^{\ell} \frac{g_{\rho}^{2}}{m_{S}^{2}}\left(\bar{f}_{L}^{i} f_{R}^{j} \bar{f}_{L}^{k} f_{R}^{\ell}\right) .
$$

Taking the lightest Higgs mass to be $m_{h}^{2} \lesssim Y_{t}^{2} v^{2}$, as it happens, for example, in the MCHM, the contribution of the first term of eq. 20 is enhanced with respect to eq. 19 by at least $\left(m_{\rho} / m_{h}\right)^{2}(v / f)^{4} \sim$ $\left(g_{\rho} / Y_{t}\right)^{2}(v / f)^{2} \gg 1$. The second term of eq. 20 is potentially even more dangerous; from eq. (6) we have $m_{S}^{2} \lesssim g_{S M}^{2} f^{2}$ that leads to a contribution larger than eq. $19 p$ by a factor $\left(m_{\rho} / m_{S}\right)^{2} \sim\left(g_{\rho} / g_{S M}\right)^{2} \gg 1$.

The group theoretical mechanism that can control the above Higgs-mediated FCNC works as follows. At the leading order, at which loops of elementary states are neglected, the $f_{L, R}$ fields and the $y_{L, R}$ spurions always enter together in the combinations (again flavor indices $i$ not shown)

$$
\left(f_{L}\right)_{\bar{\alpha}}\left(y_{L}^{* \bar{\alpha}}\right)^{I_{f_{L}}} / g_{\rho} \equiv \Psi_{L}^{I_{f_{L}}}, \quad\left(f_{R}\right)\left(y_{R}^{*}\right)^{I_{f_{R}}} / g_{\rho} \equiv \Psi_{R}^{I_{f_{R}}}
$$

In order to discuss what kind of terms will appear in eqs. (14) and (15) we have to classify all the possible operators compatible with the $G$ symmetry, with zero derivatives and any number of insertions of the NGB $\Pi$. This is best done by introducing the NGB matrix $U(\Pi)$

$$
U(\Pi)=e^{i \frac{1}{f} \Pi^{\hat{a}} T^{\hat{a}}},
$$


where $T^{\hat{a}}$ denotes the broken generators of the coset. The NGB matrix transforms as

$$
U(\Pi) \rightarrow U\left(\Pi^{(g)}\right)=g U(\Pi) h^{\dagger}(\Pi, g)
$$

where $h \in H$. As the previous equation makes manifest, via a multiplication by $U^{\dagger}$, any representation of $G$ can be "converted" into a representation of $H$. As in the standard CCWZ construction [3], then, the $G$ invariants are provided by the $H$-invariants in the tensor product $\mathbf{r}_{L} \otimes \mathbf{r}_{R}$. The most general such an invariant will read

$$
\mathcal{L}_{Y}=m_{\rho} \sum_{A, i, j} a_{i j}^{A} \bar{\Psi}_{L}^{i} U(\Pi) P_{A} U^{\dagger}(\Pi) \Psi_{R}^{j}+\text { h.c. },
$$

where $A$ indicates any $H$ invariant contained in $\mathbf{r}_{L} \otimes \mathbf{r}_{R}$, while $P_{A}$ represents the corresponding projector. Since the couplings $y_{L, R}$ break $G$, the $\Psi_{L}$ and $\Psi_{R}$ in eq. (21) are incomplete $G$ multiplets. This explicit breaking of $G$ leads, upon expansion of the above formula, to a set of Yukawa structures 1418 .

In the simplest situation, the proto-Yukawa matrices $\left(y_{L}^{i}\right)^{\bar{\alpha} I_{f_{L}}}$ for different flavors $i$ are proportional to one another, and similarly for $y_{R}^{i}$. That situation arises necessarily when, compatibly with the SM quantum numbers, there exists only one embedding of $f_{L}$ and $f_{R}$, in respectively $\mathbf{r}_{L}$ and $\mathbf{r}_{R}$. In that case the number of independent Yukawa structures is clearly bounded by the number $N$ of invariants. Notice however that for the particular case $\mathbf{r}_{L}=\mathbf{r}_{R}$, there exists one trivial invariant (corresponding to $P_{A}=1$ in eq. (24)) that does not depend on the NGB, and which will vanish when the $\Psi_{L, R}$ are put to their physical values in eq. 211 9 . In that case the number of invariants is $N-1$. Now, Higgs-mediated flavor violations are absent if the number of non-trivial invariants is 1 for both the up and the down sector. This is because in that case the flavor dependence will unavoidably factorize in eq. (24) leading to the structure of eq. (16).

The one we have just described is the simplest situation. When there exists more than one inequivalent way to embed $f_{L}$ and $f_{R}$ into respectively $\mathbf{r}_{L}$ and $\mathbf{r}_{R}$, the orientation of the matrices $\left(y_{L}^{i}\right)^{\bar{\alpha} I_{f_{L}}}$ (and similarly for $y_{R}^{i}$ ) can depend on $i$. In that case it is easy to conclude that the number of independent structures arising from eq. (24) is given by the number of non-trivial invariants times the number of independent embeddings. For instance if there are two independent embeddings for $f_{L}$ but only one for $f_{R}$ we get twice as many structures, if there are two independent embeddings in both $\mathrm{L}$ and $\mathrm{R}$ we get 4 times as many Yukawa structures. In the minimal case studied in [11] the doublet and the singlet SM fermions are embedded in a unique way in the $\mathbf{5}$ of $\mathrm{SO}(5)$. That model belongs thus to first simple class of models. On the other hand, in the composite 2HDM we will typically have multiple embeddings. We then have to force the same embedding for all flavors by either imposing a symmetry or by an ansatz, in the same spirit of MFV. Let us now see how all this works in explicit examples.

Consider first $\mathrm{SO}(6) / \mathrm{SO}(4) \times \mathrm{SO}(2)$ with $q_{L} \in \Psi_{L}=\mathbf{2 0}^{\prime}$ and $u_{R}, d_{R} \in \Psi_{R}=\mathbf{1}$. In the tensor product $\mathbf{2 0}^{\prime} \otimes \mathbf{1}$ there is obviously only one invariant, which seems already good. However $\mathbf{2 0}^{\prime}$ contains two independent $(\mathbf{2}, \mathbf{2})$, forming an $\mathrm{SO}(2)$ doublet, so that there exists two independent embeddings of $q_{L}$ into

\footnotetext{
${ }^{9}$ This is simply because no gauge invariant bilinear $\bar{f}_{L} f_{R}$ can be written without the insertion of at least one Higgs field.
} 
the $\mathbf{2 0}^{\prime}$. One of the embedding can be forbidden by demanding the coupling in eq. 17) to satisfy $C_{2}$, that is just a reflection in the 5,6 plane of $\mathrm{SO}(6)$. This leads to a composite $2 \mathrm{HDM}$ of type I. One could also fold $C_{2}$ with isospin parity $C_{I}$ (under which $\left(u_{R}, d_{R}\right) \rightarrow\left(u_{R},-d_{R}\right)$ ) and thus obtain the analogue of type II. Finally one could assume an ansatz according to which the embedding of $q_{L}$ into $\mathbf{2 0}$ ' is flavor independent. This would correspond to the composite version of type III. Of the three scenarios we outlined, the second and the third still requires an unbroken approximate $C_{1} P$ symmetry to control $\widehat{T}$.

Consider now again $\mathrm{SO}(6) / \mathrm{SO}(4) \times \mathrm{SO}(2)$ but with matter embedded as $q_{L} \in \Psi_{L}=\mathbf{6}$ and $u_{R}, d_{R} \in$ $\Psi_{R}=\mathbf{6}$. Decomposing $\mathbf{6}=\mathbf{4}+\mathbf{2} \equiv v_{4} \oplus v_{2}$ under $\mathrm{SO}(4) \times \mathrm{SO}(2)$, with an obvious notation, we find that $\mathbf{6} \otimes \mathbf{6}$ contains 3 invariants: $\left(v_{4} \cdot v_{4}\right), v_{2} \cdot v_{2}$ and $v_{2} \wedge v_{2}$. One combination, $v_{4} \cdot v_{4}+v_{2} \cdot v_{2}$ is trivial and so we are left with two non-trivial invariants, that we can choose to be $v_{4} \cdot v_{4}$ and $v_{2} \wedge v_{2}$. In order to reduce the number of possible Yukawa structures we are forced to assume the strong sector respects $C_{2}$. Then depending on the overall $C_{2}$ parities of $\mathcal{O}_{I_{f_{L}, R}}$ either $v_{4} \cdot v_{4}$ or $v_{2} \wedge v_{2}$ will be eliminated. This is not yet enough because there are two independent ways to embed $u_{R}, d_{R} \in \Psi_{R}=\mathbf{6}$, either into the 5 th or the 6th entry. This gives two possible Yukawa structures from the most general coupling in eq. (17). At this stage we can proceed like in the first model we discussed. If we assume that the mixing also respects $C_{2}$, the number of structures is just one, and obtain the analogue of type I. If we assume $C_{I} \cdot C_{2}$, we obtain the analogue of type II. And if we assume the embedding breaks $C_{2}$ while remaining flavor independent, we obtain the analogue of type III. But notice that even in this third case to eliminate one invariant we still need to assume that the strong sector respects $C_{2}$.

\subsection{Electroweak Precision Observables}

In this section we review the issue of electroweak precision tests and also take the opportunity to improve in a significant way the analysis of ref. [5].

The main advantage of PNGB Higgs models, compared to technicolor, is the possibility to tune $v$ to be somewhat smaller than the fundamental scale $f$. This permits to control dangerous corrections to electroweak observables. On the other hand, a model is the more plausible the larger $v / f$ is. Because of that, electroweak precision tests (EWPT) still constrain significantly the structure of composite Higgs models. The first obvious constraint is given by the $S$-parameter

$$
\widehat{S} \sim \frac{m_{W}^{2}}{m_{\rho}^{2}} \sim \frac{g^{2}}{g_{\rho}^{2}} \frac{v^{2}}{f^{2}} .
$$

From the experimental constraint on $\widehat{S}$, we obtain the lower bound $m_{\rho} \gtrsim 2 \mathrm{TeV}$, or equivalently [5],

$$
\xi \equiv \frac{v^{2}}{f^{2}} \lesssim 0.01 g_{\rho}^{2} \simeq \frac{1.6}{N},
$$

showing that the larger $g_{\rho}$, i.e. the smaller $N$, the smaller the needed tuning on $\xi$. That gives one sure reason for being interested in strongly coupled models. 
The other relevant constraints are associated with the top couplings. Indeed, by eq. (8), one, or both, $y_{L}$ and $y_{R}$ must be larger than $Y_{t}$, giving potentially large effects. These can however be controlled by specific choices of the quantum numbers of the operators $\mathcal{O}_{f_{L}}$ and $\mathcal{O}_{f_{R}}$ in eq. (17). It is instructive to first just focus on the $\mathrm{SO}(4) \times \mathrm{U}(1)_{X}$ quantum numbers. Later we shall discuss the important changes due to the additional constraining power of $G / H$. For the choice $\mathcal{O}_{L}=(\mathbf{2}, \mathbf{1})_{\mathbf{1} / \mathbf{6}}, \mathcal{O}_{R}=(\mathbf{1}, \mathbf{2})_{\mathbf{1} / \mathbf{6}}$ the expected corrections to $Z \bar{b} b$ and $\widehat{T}$ are

$$
\frac{\delta g_{b}}{g_{b}} \sim \frac{y_{L}^{2}}{g_{\rho}^{2}} \xi, \quad \quad \widehat{T} \sim \frac{N_{c} y_{R}^{4}}{16 \pi^{2} g_{\rho}^{2}} \xi .
$$

Notice that for our choice of embedding, $y_{L}$ is an isospin singlet while $y_{R}$ is a spurion of custodial isospin $1 / 2$. Since $\widehat{T}$ corresponds to a violation of 2 units of isospin charge, selection rules dictate the four powers of $y_{R}$ in eq. (27). Now, the experimental bounds, together with eq. (8) imply $\xi<0.05$. This tight bound arises because $\delta g_{b} / g_{b}$ demands a small $y_{L}, \widehat{T}$ demands a small $y_{R}$, while the two couplings are constrained to have a sizable product to reproduce $Y_{t}$. A less constrained, and thus less tuned scenario, can arise in the case where $\mathcal{O}_{L}=(\mathbf{2}, \mathbf{2})_{\mathbf{2} / \mathbf{3}}, \mathcal{O}_{R}=(\mathbf{1}, \mathbf{1})_{\mathbf{2} / \mathbf{3}}$. We also should mention that in this case to generate the Yukawas of the down sector, assuming that the right chiralities couple to a $(\mathbf{1}, \mathbf{1})_{-\mathbf{1} / \mathbf{3}}$, we need to couple the quark doublet to a second operator in the $(2,2)_{-1 / 3}{ }^{10}$. This might in general give rise to flavor problems which can be avoided with appropriate UV assumptions, see [13]. Now $y_{R}$ is an $\mathrm{SO}(4)$ singlet under the custodial group and drops out of eq. (27). However $y_{L}$ transforms as $(\mathbf{1}, \mathbf{2})$ under $\operatorname{SO}(4)$ and therefore one generically expects

$$
\frac{\delta g_{b}}{g_{b}} \sim \frac{y_{L}^{2}}{g_{\rho}^{2}} \xi, \quad \quad \widehat{T} \sim \frac{N_{c} y_{L}^{4}}{16 \pi^{2} g_{\rho}^{2}} \xi .
$$

This result is more encouraging: for $y_{L} \sim Y_{t}$ and $y_{R} \sim g_{\rho}$ corresponding to a fully composite $t_{R}$, the bound from $\delta g_{b} / g_{b}$ is comparable to the one from $\widehat{S}$, while the one from $\widehat{T}$ is much less severe.

The situation might even be better though. It was pointed out in ref. [14] that when the strong sector is invariant under $\mathrm{O}(4)=\mathrm{SO}(4) \times P_{L R}$ and not just $\mathrm{SO}(4)$, the contribution to $\delta g_{b} / g_{b}$ in eq. (28) vanishes. That result can be understood as follows. Working at lowest order in $g_{S M}$, that amounts to treating the $\mathrm{SM}$ fields as external sources, the strong sector is an exact $\mathrm{O}(4) \times \mathrm{U}(1)_{X} / \mathrm{O}(3) \times \mathrm{U}(1)_{X}$ coset. Moreover, in the same limit we can neglect $m_{W}$ as compared to $m_{\rho}$. This amounts to computing the vertices of the vector bosons at zero momentum transfer, where they can be identified with the charges of the currents in $\mathrm{O}(4) \times \mathrm{U}(1)_{X}$. In particular the coupling to the neutral vectors is given by

$$
g W_{\mu}^{3} J_{L}^{3 \mu}+g^{\prime} B_{\mu}\left(J_{R}^{3 \mu}+J_{X}^{\mu}\right) \equiv g W_{\mu}^{3}\left(J_{V}^{3 \mu}-J_{A}^{3 \mu}\right)+g^{\prime} B_{\mu}\left(J_{V}^{3 \mu}+J_{A}^{3 \mu}+J_{X}^{\mu}\right) .
$$

Now, the only correction to the current can come from the $J_{A}$ contribution, since $J_{V}$ and $J_{X}$ are conserved. However, on eigenstates of $P_{L R}$ the expectation value of the axial charge $Q_{A}^{3}$ clearly vanishes as $Q_{A}^{3}$ is odd. For these states $J_{A}^{3}$ does not contribute to the vector boson vertex, and in particular the coupling to

\footnotetext{
${ }^{10}$ We do not consider the possibility that down right-handed quarks couple to a $(\mathbf{1}, \mathbf{3})_{\mathbf{2} / \mathbf{3}}$ representation.
} 
the $Z$ is unaffected. Now in the fermion multiplet $(\mathbf{2}, \mathbf{2})_{\mathbf{2} / \mathbf{3}}$ the only eigenstate of $P_{L R}$ has electric charge $-1 / 3$, and plays the role of the bottom quark. This discussion can be complemented by an explicit effective Lagrangian analysis that makes full use of the $\mathrm{SO}(4) / \mathrm{SO}(3)$ CCWZ construction [3]. Starting from a chiral fermion $Q_{A}$ transforming like $(\mathbf{2}, \mathbf{2})$, and using the NGB matrix $U_{A \bar{A}}$, we can form the dressed fermions $\psi_{i}=Q_{A} U_{A i}^{*}(i=1,2,3)$ transforming like a $\mathbf{3}$ of $\mathrm{SO}(3)$ and $\eta=Q_{A} U_{A 4}^{*}$ transforming like a singlet. Then it is straightforward to write all the possible interactions at lowest derivative order

$$
\begin{array}{llrl}
\mathcal{O}_{1} & =\bar{\psi} \bar{\sigma}^{\mu}\left(\partial_{\mu}+\mathcal{E}_{\mu}\right) \psi & \mathcal{O}_{2}=\bar{\eta} \bar{\sigma}^{\mu} \partial_{\mu} \eta \\
\mathcal{O}_{3}=\bar{\psi}_{i} \bar{\sigma}^{\mu} \eta \mathcal{D}_{i \mu} & \mathcal{O}_{4}=\bar{\psi}_{i} \bar{\sigma}^{\mu} \psi_{j} \mathcal{D}_{k \mu} \epsilon_{i j k}
\end{array}
$$

where $\mathcal{E}_{\mu}$ and $\mathcal{D}_{\mu}$ are the $H$ connection and $G / H$ NGB respectively [3]. $\mathcal{O}_{1,2,3}$ are manifestly $P_{L R}$ invariant, and give no correction to $g_{b}$ upon weak gauging of the SM group. On the other hand $\mathcal{O}_{4}$ breaks $P_{L R}$ and does indeed renormalize $g_{b} 11$.

Now, what is remarkable, and was indeed missed in [5], is that when the Higgs scalar is itself a NGB residing into a bigger coset such as $\mathrm{SO}(5) / \mathrm{SO}(4)$ or $\mathrm{SO}(6) / \mathrm{SO}(4) \times \mathrm{SO}(2)$ the $P_{L R}$ arises as an accidental symmetry of the lowest derivative interactions. This is very similar to the case of $C_{2}$, an accidental symmetry of the 2-derivative $\sigma$-model. It is easy to prove that by extending the previous analysis to $\mathrm{SO}(5) / \mathrm{SO}(4)$ and assuming $\mathcal{O}_{L}=\mathbf{5}_{2 / 3}$. The corresponding fermion is $Q_{A}$, with $A=1, \ldots, 5$. Dressing it with NGB, we obtain $\psi_{i}=Q_{A} U_{A i}^{*}(i=1,2,3,4)$ transforming like a 4 of $\mathrm{SO}(4)$ and the singlet $\eta=Q_{A} U_{A 5}^{*}$. Now we can still write the same $P_{L R}$ invariant contractions corresponding to $\mathcal{O}_{1,2,3}$. However, at the one derivative level we cannot write the analogue of $\mathcal{O}_{4}$ since the Levi-Civita tensor of $\mathrm{SO}(4)$ has four indices! One can easily extend this analysis to $\mathrm{SO}(6) / \mathrm{SO}(4) \times \mathrm{SO}(2)$ with $\mathcal{O}_{L}$ either in the $\mathbf{6}$ or $\mathbf{2 0}$. Again the main point is the impossibility of writing invariants that involve the Levi-Civita tensor.

In view of the latter result, in all the cases considered in previous literature $\mathrm{SO}(5) / \mathrm{SO}(4)$ or $\mathrm{SO}(6) / \mathrm{SO}(5)$ and in all the models studied in the present paper, experimental constraints allow a sizeable $y_{L}>Y_{t}$. Indeed the bound on $\widehat{T}$ (and also that on $B-\bar{B}$ mixing) can be met for $y_{L}$ as big as roughly $y_{L} \sim \sqrt{Y_{t} g_{\rho}}$. In that case a Higgs boson as heavy as $300 \mathrm{GeV}$ could be obtained.

\section{Explicit Models}

It is not difficult, making use of the general considerations outlined in the previous section, to construct potentially realistic scenarios with two composite PNGB Higgs doublets. The aim of the present section is to describe few examples that will be classified, as in section 2.2 , in terms of the extra symmetry which will be assumed in order to deal with the $\widehat{T}$ constraint. The case of discrete symmetries $\left(C_{1} P\right.$ or $\left.C_{2}\right)$ will be considered below, restricting for definiteness to the $\mathrm{SO}(6) / \mathrm{SO}(4) \times \mathrm{SO}(2)$ coset, while the possibility of an extended global custodial group will be explored in section 3.2 . Each scenario will be defined by its $G / H$

\footnotetext{
${ }^{11}$ In the analysis of ref. [14] only three operators are mentioned. The fourth operator left out is just the trivial kinetic term $\bar{Q} \bar{\sigma}_{\mu} \partial^{\mu} Q$ invariant under the linearly realized $\mathrm{O}(4)$ and corresponding to a linear combination of $\mathcal{O}_{1,2,3}$.
} 
coset, by extra discrete symmetries if needed, and by the SM fermion's embeddings into $G$ representations, i.e. the $G$ representations of the operators to which the SM fermions are assumed to mix. Within each model, the flavor structure will be described according to the general rules of section 2.3 . Also, we will study the structure of the Higgs potential which, as we will see, is almost completely under control if extra assumptions are made on the $G$-breaking couplings external to the strong-sector. We will work under the rather strong assumption, dictated however by minimality, that the only sources of $G$-breaking are those unavoidably present, i.e. the SM gauge couplings and the fermion's couplings. This will allow us to parametrize the Higgs potential, at each given order in the gauge and fermion couplings, in terms of a limited number of coefficients and to check if they allow for EWSB and the mild tuning eq. (26). We will also derive, in some specific model, interesting consequences on the spectrum of the physical Higgs scalars.

\section{$3.1 \quad \mathrm{SO}(6) / \mathrm{SO}(4) \times \mathrm{SO}(2)$ Models}

To set the notation we will use the following basis for the generators (in the fundamental representation) of $\mathrm{SO}(6)$ algebra,

$$
\begin{aligned}
& \left(T_{R}^{a}\right)_{I J}=\frac{i}{2}\left[\frac{1}{2} \epsilon^{a b c}\left(\delta_{I}^{b} \delta_{J}^{c}-\delta_{J}^{b} \delta_{I}^{c}\right)+\left(\delta_{I}^{a} \delta_{J}^{4}-\delta_{J}^{a} \delta_{I}^{4}\right)\right](-1)^{\delta_{2}^{a}} \\
& \left(T_{L}^{a}\right)_{I J}=\frac{i}{2}\left[\frac{1}{2} \epsilon^{a b c}\left(\delta_{I}^{b} \delta_{J}^{c}-\delta_{J}^{b} \delta_{I}^{c}\right)-\left(\delta_{I}^{a} \delta_{J}^{4}-\delta_{J}^{a} \delta_{I}^{4}\right)\right](-1)^{\delta_{1}^{a}} \\
& \left(T_{S}\right)_{I J}=-\frac{i}{\sqrt{2}}\left(\delta_{I}^{5} \delta_{J}^{6}-\delta_{J}^{5} \delta_{I}^{6}\right), \\
& \left(T_{\widehat{1}}^{i}\right)_{I J}=-\frac{i}{\sqrt{2}}\left(\delta_{I}^{i} \delta_{J}^{5}-\delta_{J}^{i} \delta_{I}^{5}\right), \\
& \left(T_{\widehat{2}}^{i}\right)_{I J}=-\frac{i}{\sqrt{2}}\left(\delta_{I}^{i} \delta_{J}^{6}-\delta_{J}^{i} \delta_{I}^{6}\right),
\end{aligned}
$$

where $I, J=1, \ldots, 6, i=1, \ldots, 4$ and $a=1, \ldots, 3$. The generators $T_{R, L}^{a}$ and $T_{S}$ represent, respectively, the $\mathrm{SO}(4) \cong \mathrm{SU}(2)_{L} \times \mathrm{SU}(2)_{R}$ and $\mathrm{SO}(2)$ subgroups while the $\mathrm{SO}(6) / \mathrm{SO}(4) \times \mathrm{SO}(2)$ coset is spanned by $T_{\alpha}^{i}$, with $\alpha=\widehat{1}, \widehat{2}$. The broken generators $T_{\alpha}^{i}$ are associated with the NGB, transforming as a $(4,2)$ of $\mathrm{SO}(4) \times \mathrm{SO}(2)$. Consistently with table 1 we therefore see that the coset delivers two $\mathrm{NGB} \mathrm{SU}(2)_{L}$-doublets $\Phi^{\alpha}=\left(\Phi^{\widehat{1}}, \Phi^{\widehat{2}}\right)$.

As in section 2.3, to derive the constraints from $\mathrm{SO}(6)$ symmetry we will introduce the NGB matrix $U(\Pi)$ transforming as in eq. (23). We will mostly use the fundamental representation $U^{6}$. Using (32), the NGB matrix is given explicitly by

$$
\left(U^{\mathbf{6}}\right)_{\bar{I}}^{I}=\left(e^{i \frac{\sqrt{2} \Pi}{f}}\right)_{\bar{I}}^{I}, \quad \Pi=T_{\alpha}^{i} \Phi_{i}^{\alpha}=\frac{i}{\sqrt{2}}\left(\begin{array}{c|c}
0_{4 \times 4} & \Phi^{\widehat{1}} \Phi^{\widehat{2}} \\
\hline-\Phi^{\hat{1}} & 0_{2 \times 2} \\
-\Phi^{\widehat{2}} & 0^{2}
\end{array}\right),
$$

where the index $I$ is in the fundamental of $\mathrm{SO}(6)$ while the index $\bar{I}$ transforms in a reducible (non-linear) representation of $\mathrm{SO}(6)$, that is through multiplication by the matrix $h$ in eq. (23). In the case of the 
6 representation, $h$ is composed of two blocks, corresponding respectively to an $\mathrm{SO}(4)$ and to an $\mathrm{SO}(2)$ rotation. The index $\bar{I}$ therefore runs over two components, $\bar{I} \equiv\{i, \alpha\}$, such that $i$ labels the components of an $\mathrm{SO}(4)$ 4-plet while $\alpha$ labels the components of an $\mathrm{SO}(2)$ doublet. Besides the global $\mathrm{SO}(6)$ group, we will also be interested in discrete symmetries and in particular in the $C_{1,2}$ parities defined in section 2.2 . The matrix $U^{\mathbf{6}}$ transforms as $U^{\mathbf{6}} \rightarrow \mathcal{C}_{1,2}^{\mathbf{6}} \cdot U^{\mathbf{6}} \cdot \mathcal{C}_{1,2}^{\mathbf{6}}$ with

$$
\mathcal{C}_{1}^{\mathbf{6}}=\operatorname{diag}(-1,1,-1,1,1,1), \quad \mathcal{C}_{2}^{\mathbf{6}}=\operatorname{diag}(1,1,1,1,1,-1) .
$$

We see that $C_{1}$ is an element of the $\mathrm{SO}(4)$ unbroken subgroup and that $C_{2}$ acts as parity in 6 dimensions, defined as the inversion of the last coordinate, on both the $I$ and $\bar{I}$ indices. Notice that an appropriate NGB matrix $U^{\mathbf{r}}$ might be defined for each $\mathrm{SO}(6)$ representation $\mathbf{r}$. For vectorial representations, such as the $\mathbf{2 0}^{\prime}$ we will use below, $U^{\mathbf{r}}$ is trivially obtained in terms of products of $U^{\mathbf{6}}$.

\subsubsection{Higgs Potential}

The Higgs potential originates from the $\mathrm{SO}(6)$ breaking effects, which we have assumed to be only due to the $\mathrm{SU}(2)_{L} \times \mathrm{U}(1)_{Y}$ gauge and fermion couplings. Among the latter, only those associated to the top quark mass will give a sizable contribution and will be considered in what follows. The structure will be determined by the $\mathrm{SO}(6)$ representations $\mathbf{r}_{Q, T}$ to which the $q_{L}=\left(t_{L}, b_{L}\right)$ and $t_{R}$ doublet and singlet are coupled to

$$
\mathcal{L}_{\text {mix }}=\left(\bar{q}_{L}\right)_{\bar{\alpha}}\left(y_{L}^{\bar{\alpha}}\right)^{I_{Q}} \mathcal{O}_{I_{Q}}+\left(\bar{t}_{R}\right)\left(y_{R}\right)^{I_{T}} \mathcal{O}_{I_{T}}+\text { h.c. . }
$$

As in the discussion below eq. (17), the implications of the symmetries can be worked out regarding the $y$ 's as non-dynamical external spurionic fields. The $I_{Q, T}$ indices are, respectively, in the $\mathbf{r}_{Q, T}$ representations of the $\mathrm{SO}(6)$ symmetry group of the strong sector, while $\bar{\alpha}=1,2$ are indices of the "elementary" $\mathrm{U}(2)_{L}^{\mathrm{el}}$ group under which the $q_{L}^{\bar{\alpha}}$ rotate, the strong sector and in particular the Higgs fields being invariant ${ }^{12}$, A second elementary group, under which $y_{R}$ is charged, is the $\mathrm{U}(1)_{R}^{\mathrm{el}}$ of $t_{R}$. Given that the Higgs is neutral, requiring the potential to be invariant under these additional elementary symmetries forces it to depend on $y_{L, R}$ only via the combinations:

$$
\begin{aligned}
& \left(\Upsilon_{L}\right)^{I_{Q} J_{Q}}=\left(y_{L}^{*} \bar{\alpha}\right)^{I_{Q}}\left(y_{L}^{\bar{\alpha}}\right)^{J_{Q}}, \\
& \left(\Upsilon_{R}\right)^{I_{T} J_{T}}=\left(y_{R}^{*}\right)^{I_{T}}\left(y_{R}\right)^{J_{T}} .
\end{aligned}
$$

In the small-coupling expansion, making use of the power counting rule described in section 2.1, the Higgs potential at one loop order takes the form

$$
V=\frac{m_{\rho}^{4}}{16 \pi^{2}} \sum_{n_{R}, n_{L}} \frac{1}{\left(g_{\rho}^{2}\right)^{n_{R}+n_{L}}} \sum_{\delta} c_{\delta}^{\left(n_{R}, n_{L}\right)} \mathcal{I}_{\left(n_{R}, n_{L}\right)}^{\delta},
$$

\footnotetext{
${ }^{12}$ According to this formal viewpoint, the "expectation" values of the external spurions $y_{L, R}$ and $g$ break the fictitious extended symmetry to a diagonal $\mathrm{SU}(2) \times \mathrm{U}(1)$ under which the Higgs multiplets have their usual quantum numbers.
} 
where $\mathcal{I}_{\left(n_{R}, n_{L}\right)}^{\delta}$ denotes $\mathrm{SO}(6)$ invariant operators constructed with the NGB and $n_{R, L}$ powers of $\Upsilon_{R, L}$, while $c_{\delta}^{\left(n_{R}, n_{L}\right)}$ are order one coefficients.

It is straightforward to classify these invariants at each given order proceeding similarly to section 2.3 The central objects are the dressed spurions $\bar{\Upsilon}_{L, R}$,

$$
\begin{aligned}
& \left(\bar{\Upsilon}_{L}\right)^{\bar{I} \bar{J}} \equiv\left(U^{\mathbf{r}_{Q} \dagger}\right)_{I}^{\bar{I}}\left(U^{\mathbf{r}_{Q} \dagger}\right)_{J}^{\bar{J}}\left(\Upsilon_{L}\right)^{I J} \\
& \left(\bar{\Upsilon}_{R}\right)^{\bar{I} \bar{J}} \equiv\left(U^{\mathbf{r}_{T} \dagger}\right)_{I}^{\bar{I}}\left(U^{\mathbf{r}_{T}^{\dagger}}\right)_{J}^{\bar{J}}\left(\Upsilon_{R}\right)^{I J},
\end{aligned}
$$

obtained by rotating $\Upsilon_{L, R}$ with the NGB matrix in the appropriate representation. Because of eq. 23, and by the same argument we made in the previous section concerning the $\bar{I}$ index in $\mathbf{6}$, the $\bar{\Upsilon}_{L, R}$ form a reducible non-linear representation of $\mathrm{SO}(6)$. More explicitly, they transform as

$$
\left(\bar{\Upsilon}_{L, R}\right)^{\overline{I J}} \rightarrow\left(h^{\mathbf{r}_{Q, T}}(\Phi, g)\right)^{\bar{I}}{ }_{\bar{K}}\left(h^{\mathbf{r}_{Q, T}}(\Phi, g)\right)^{\bar{J}}{ }_{L}\left(\bar{\Upsilon}_{L, R}\right)^{\bar{K} \bar{L}}
$$

where $h^{\mathbf{r}_{Q, T}}$ takes, as before, a block-diagonal form. To construct the $\mathrm{SO}(6)$ invariants we therefore simply have to classify all possible $\mathrm{SO}(4) \times \mathrm{SO}(2)$ invariants that can be built out of $\bar{\Upsilon}_{L, R}$ at a given order. Notice that among the $\mathrm{SO}(4) \times \mathrm{SO}(2)$ invariants, the special ones that are also invariant under $\mathrm{SO}(6)$ are clearly not interesting. Indeed, by the definition (38), $U^{\mathbf{r}}$ cancels out when forming $\mathrm{SO}(6)$ invariants, which therefore give a field independent constant contribution to the potential.

It will be relevant in our classification to establish the $C_{2}$ and $C_{1} P$ parities of each invariant. Given that the SM elementary fermions are $C_{2}$ even, invariance of $\mathcal{L}_{\text {mix }}$ in eq. (35) is ensured by formally assigning the following $C_{2}$ transformation

$$
\left(y_{L, R}\right)^{I} \rightarrow\left(\mathcal{C}_{2}^{\mathbf{r}_{Q, T}}\right)^{I}{ }_{J}\left(y_{L, R}\right)^{J} \Rightarrow\left(\bar{\Upsilon}_{L, R}\right)^{\overline{I J}} \rightarrow\left(\mathcal{C}_{2}^{\mathbf{r}_{Q, T}}\right)^{\bar{I}}{ }_{\bar{K}}\left(\mathcal{C}_{2}^{\mathbf{r}_{Q, T}}\right)^{\bar{J}}\left(\bar{\Upsilon}_{L, R}\right)^{\bar{K} \bar{L}}
$$

where $\mathcal{C}_{2}^{\mathbf{r}_{Q, T}}$ denotes the $C_{2}$ action in the appropriate representations. For vector-like representations this is again easily obtained from the one in the fundamental, $\mathcal{C}_{2}^{6}$, which is reported in eq. (34). For what concerns the action of $C_{1} P$ (see section 2.2), it coincides with "ordinary" $C P$ on the elementary fermions, on the SM gauge fields and on the Higgs. This last requirement fixes $C_{1} P$ to act on the NGB matrix as parity $(\vec{x} \rightarrow-\vec{x})$ combined with the $C_{1}$ transformation defined in eq. (34). On the fermionic operators of the strong sector, such as the ones that mix with the elementary fermions, we take $C_{1} P$ to be ordinary $C P$, $\mathcal{O} \rightarrow \overline{\mathcal{O}}$ in Weyl notation, combined with the $C_{1}$ transformation that we have introduced for the Higgs. Invariance of eq. (35) implies for the couplings the following transformation

$$
\left(y_{L, R}\right)^{I} \rightarrow\left(\mathcal{C}_{1}^{\mathbf{r}_{Q, T}}\right)_{J}^{I}\left(y_{L, R}^{*}\right)^{J}
$$

Remember however that $\mathcal{C}_{1}$ is an element of the symmetry group of the strong sector, and that the $\mathcal{I}^{\delta}$, $\mathrm{s}$ are automatically invariant under such transformations. For the purpose of establishing the $C_{1} P$ parities 
of the various invariants, the $\mathcal{C}_{1}$ part of the transformation can therefore be ignored and the action of $C_{1} P$ effectively reduces to $y_{L, R} \rightarrow y_{L, R}^{*}$ or, even more simply

$$
\left(\bar{\Upsilon}_{L, R}\right)^{\overline{I J}} \rightarrow\left(\bar{\Upsilon}_{L, R}\right)^{\overline{J I}}
$$

Let us now apply these general considerations to two specific choices of the $\mathbf{r}_{Q, T}$ representations that will be useful in the following: $\mathbf{r}_{Q, T}=\mathbf{6}$ and $\left\{\mathbf{r}_{Q}, \mathbf{r}_{T}\right\}=\left\{\mathbf{2 0}^{\prime}, \mathbf{1}\right\}$.

In our discussion we shall call spurionic the symmetries that are formally satisfied by the effective action when the spurions are tranformed according to the rules we discussed. We shall instead call residual the symmetries that are truly unbroken, that is when the spurions are not tranformed.

\section{Fermion contributions with $\mathbf{r}_{Q, T}=6$ :}

Both $q_{L}$ and $t_{R}$ couple, respecting $\mathrm{SU}(2)_{L} \times \mathrm{U}(1)_{Y}$, to fermionic operators in the 6 with $X=2 / 3$ (as usual the hypercharge is given by $\left.Y=T_{R}^{3}+X\right)$. More precisely $q_{L}$ couples to the $\mathbf{4}_{\mathbf{2} / \mathbf{3}}$ of $\mathrm{SO}(4) \times \mathrm{U}(1)_{X}$ which populates the first 4 entries of the $\mathbf{6}_{\mathbf{2} / \mathbf{3}}$. Given that there is a unique embedding of $q_{L}$ in the $\mathbf{4}_{\mathbf{2} / \mathbf{3}}$, the physical value of the $y_{L}$ spurion in eq. (35) which determines the $q_{L}$ coupling is uniquely fixed to be

$$
\left(y_{L}^{\bar{\alpha}}\right)^{I}=\frac{y_{L}}{\sqrt{2}}\left\{\left(\vec{v}^{\overline{1}}, 0,0\right),\left(\vec{v}^{\overline{2}}, 0,0\right)\right\}
$$

where $y_{L}$ has been made real by an $\mathrm{U}(1)$ rotation of the elementary $q_{L}$ and we have defined the vectors,

$$
\begin{aligned}
\vec{v}^{\overline{1}} & =(0,0, i,-1), \\
\vec{v}^{\overline{2}} & =(-i, 1,0,0) .
\end{aligned}
$$

We see, comparing with eqs. 4041), that the $y_{L}$ 's VEV is automatically invariant under both $C_{1} P$ and $C_{2}$. Provided one of the two parities was a symmetry of the strong sector, $y_{L}$ will not induce new breaking effects. The situation is different for $y_{R}$. Given that the 6 (with, again, $X=2 / 3$ ) contains two $\mathrm{SU}(2)_{L}$ singlets with the hypercharge of the $t_{R}$, the most general form of its VEV is

$$
\left(y_{R}\right)^{I}=\left(0,0,0,0, \vec{v}_{R}+i \vec{v}_{I}\right),
$$

where $\vec{v}_{R, I}$ are two real $\mathrm{SO}(2)$ vectors. By combining an $\mathrm{SO}(2)$ strong sector's rotation with a $\mathrm{U}(1)_{R}^{\mathrm{el}}$ phase transformation, $\vec{v}_{R}$ and $\vec{v}_{I}$ can be aligned respectively along $(1,0)$ and $(0,1)$, allowing to parametrize the most general VEV of the $y_{R}$ spurion as

$$
\left(y_{R}\right)^{I}=y_{R}(0,0,0,0, \cos \theta, i \sin \theta) .
$$

with $y_{R}$ real. This general VEV, looking again at eqs. 40[41), breaks both $C_{1} P$ and $C_{2}$ while it preserves the product $C_{1} P \cdot C_{2}$. Both $C_{1} P$ and $C_{2}$ are preserved in the special case $\theta=0$.

Let us now proceed, following the general method outlined before, to the classification of the possible contributions to the Higgs potential. The $\bar{\Upsilon}_{L, R}$ have two indices in the $\mathbf{6}$, which decompose as $(\mathbf{4}, \mathbf{1}) \oplus(\mathbf{1}, \mathbf{2})$ 
under $\mathrm{SO}(4) \times \mathrm{SO}(2)$. At leading order $\left(n_{L, R}=1\right)$ six $\mathrm{SO}(4) \times \mathrm{SO}(2)$ invariants can be formed, three from $\bar{\Upsilon}_{L}$ and three from $\bar{\Upsilon}_{R}$. One linear combination of each of these three, corresponding to the $\mathrm{SO}(6)$ invariant $\left(\bar{\Upsilon}_{L, R}\right)^{\overline{I J}} \delta_{\overline{I J}}$, is independent of the Higgs field and must be removed from the counting. We are therefore left with four operators

$$
\begin{array}{ll}
\mathcal{I}_{(1,0)}^{1}=\delta_{i j}\left(\bar{\Upsilon}_{R}\right)^{i j}, & \mathcal{I}_{(0,1)}^{1}=\delta_{i j}\left(\bar{\Upsilon}_{L}\right)^{i j}, \\
\mathcal{I}_{(1,0)}^{2}=\epsilon_{\alpha \beta}\left(\bar{\Upsilon}_{R}\right)^{\alpha \beta}, & \mathcal{I}_{(0,1)}^{2}=\epsilon_{\alpha \beta}\left(\bar{\Upsilon}_{L}\right)^{\alpha \beta},
\end{array}
$$

the first two are even under both $C_{1} P$ and $C_{2}$ while the others are odd, as summarized in table 2 . The parities we refer to are the spurionic ones, obtained by transforming the spurions as in eqs. 40 41] independently on whether their VEV preserves the symmetry or not. Under the assumption that the strong sector is invariant under either $C_{1} P$ or $C_{2}$, the spurionic parities determine whether a given operator can be generated or not.

After the invariants are classified and written explicitly, the last step which is needed in order to compute their actual contribution to the Higgs potential is to substitute the spurions with their VEVs, which are given by eqs. 43 46). Given that the VEV of eq. (46) breaks (for $\theta \neq 0$ ) $C_{1} P$ and $C_{2}$, the parities of the corresponding contributions to the potential, which we denote as residual parities, do not coincide, in general, with the spurionic ones. These residual parities are shown in table 2 notice that not all the operators have a definite residual parity because substituting a parity-breaking spurion VEV might make the operator acquire one parity-even and one parity-odd part. From the table we can also read if each operator, again after the spurions have taken their $\mathrm{VEV}$, is invariant under the $\mathrm{SO}(4)$ custodial symmetry or not. Given that custodial is only broken by the $y_{L}$ spurions, it comes as no surprise that it is preserved by $\mathcal{I}_{(1,0)}^{1}$ and $\mathcal{I}_{(1,0)}^{2}$ while it is broken by $\mathcal{I}_{(0,1)}^{2}$. The invariance of $\mathcal{I}_{(0,1)}^{1}$ is more surprising and it can be understood as follows. Whenever the $q_{L}$ couples to a 4 of $\mathrm{SO}(4)$ as in the present case (and in the one of $\left\{\mathbf{r}_{Q}, \mathbf{r}_{T}\right\}=\left\{\mathbf{2 0}^{\prime}, \mathbf{1}\right\}$ discussed in the following paragraph), the custodial-breaking part of the spurion multiplet is

$$
\left(y_{L}^{\bar{\alpha}}\right)^{i}=\frac{y_{L}}{\sqrt{2}}\left\{\vec{v}^{\overline{1}}, \vec{v}^{\overline{2}}\right\}
$$

and, because of $\mathrm{U}(2)_{L}^{\mathrm{el}}$ invariance, it will only enter through the combination

$$
\left(\Upsilon_{L}\right)^{i j}=\left(y_{L}^{*} \bar{\alpha}\right)^{i}\left(y_{L}^{\bar{\alpha}}\right)^{j}=\frac{y_{L}^{2}}{2}\left(\begin{array}{cccc}
1 & i & 0 & 0 \\
-i & 1 & 0 & 0 \\
0 & 0 & 1 & i \\
0 & 0 & -i & 1
\end{array}\right)_{i j} \equiv\left(\Upsilon_{L}^{+}\right)^{i j}+i\left(\Upsilon_{L}^{-}\right)^{i j}
$$

Here $\Upsilon_{L}^{ \pm}$denote, respectively, the symmetric and antisymmetric components of $\Upsilon_{L}$ that are even and odd under $C_{1} P$ because of eq. 42 . The VEV of $\Upsilon_{L}^{+}$, as eq. 49 shows, is proportional to the identity and therefore does not break custodial. We then understand why, at the leading order where a single power of $\Upsilon_{L}^{ \pm}$can be used to construct the potential, custodial breaking can only appear in a $C_{1} P$-odd term. That 
the latter is also $C_{2}$-odd is instead a peculiarity of the case under consideration and cannot be understood in general terms.

It is a simple exercise to continue the classification for the second order terms. The results are presented in table 2. The only new subtlety, which is first encountered at this order, concerns the coefficient of the operators, whose estimate does not always coincide with eq. (37). This is because eq. (37) assumes all the operators to be generated by one single loop of the elementary fermions (from which the $1 / 16 \pi^{2}$ prefactor), while many operators in table 2 start being generated at the two-loop level. The latter operators are formally subleading even though in practice the suppression might be small, being $O\left(g_{\rho}^{2} / 16 \pi^{2}\right)$.

Let us summarize the main results of this classification, that will be further discussed in the following. At leading order, $\propto y^{2}$, all the operators are either even or odd under both the discrete symmetries $C_{1} P$ and $C_{2}$, so that, assuming the strong sector to respect one discrete symmetry, automatically implies the other. Moreover, all the even operators will respect $\mathrm{SO}(4), C_{1} P$ and $C_{2}$ after the spurions will acquire VEVs, in spite of the fact that all these symmetries were broken by the spurion's VEVs. This leaves many accidental symmetries in the Higgs potential. Notice also that, even when it is not preserved by the strong sector, $C_{1} P \cdot C_{2}$ arises as an accidental spurionic symmetry of the potential at leading order. Given that $C_{1} P \cdot C_{2}$ is also preserved by the $\mathrm{VEV}$ of the spurions, it will therefore remain as an accidental residual symmetry of the leading order potential. These features are lost at order $y^{4}$. Indeed, two operators, $\mathcal{I}_{(2,0)}^{5} \&$ $\mathcal{I}_{(1,1)}^{2}$, break $C_{1} P \cdot C_{2}$ and $\mathrm{SO}(4)$ is broken by even operators $\left(\mathcal{I}_{(1,1)}^{1}, \mathcal{I}_{(0,2)}^{1}, \ldots\right)$. However, in the particular situation we will consider below, where the spurion VEV respects both $C_{1} P$ and $C_{2}(\theta=0)$, at order $y^{4}$ it remains true that $C_{2}$ invariance of the strong sector implies an accidental $C_{1} P$ in the potential.

Fermion contributions with $\left\{\mathbf{r}_{Q}, \mathbf{r}_{T}\right\}=\left\{\mathbf{2 0}^{\prime}, \mathbf{1}\right\}$ :

The $\mathbf{2 0}^{\prime}$ representation is the symmetric and traceless product of two $\mathbf{6}$, and it decomposes under $\mathrm{SO}(4) \times$ $\mathrm{SO}(2)$ as

$$
\mathbf{2 0}^{\prime}=(\mathbf{9}, \mathbf{1}) \oplus(\mathbf{4}, \mathbf{2}) \oplus(\mathbf{1}, \mathbf{2}) \oplus(\mathbf{1}, \mathbf{1}) .
$$

Operators in this representation and $X=2 / 3$ can be coupled to $q_{L}$ as in the case of the 6 . Unlike that case, however, we now have two four-plets of $\mathrm{SO}(4)$ to which the doublet could mix. The $y_{L}$-spurion's VEV is therefore not uniquely determined in general. Assuming the VEV to be either $C_{1} P$ or $C_{2}$ invariant uniquely fixes the embedding,

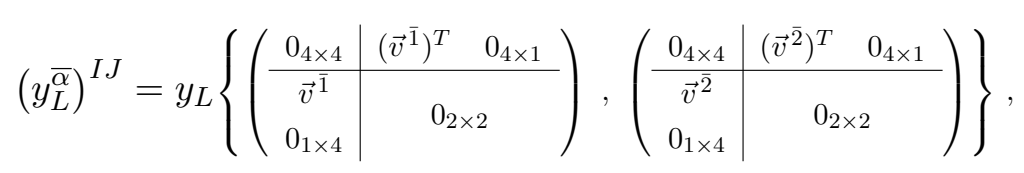

so that, as for the $y_{R}$ spurion in the previous $\{\mathbf{6}, \mathbf{6}\}$ case, imposing the VEV to respect one of the symmetries automatically implies the other. Unlike for the $\{\mathbf{6}, \mathbf{6}\}$, we will only consider the $C_{2}, C_{1} P$ symmetric yukawa of eq. 51 . Out of $y_{L}$ we build $\left(\bar{\Upsilon}_{L}\right)^{I J K L}$, which has now four indices, and classify the $\mathrm{SO}(4) \times \mathrm{SO}(2)$ invariants. Fortunately, as discussed in the following section, the leading order terms will be sufficient 


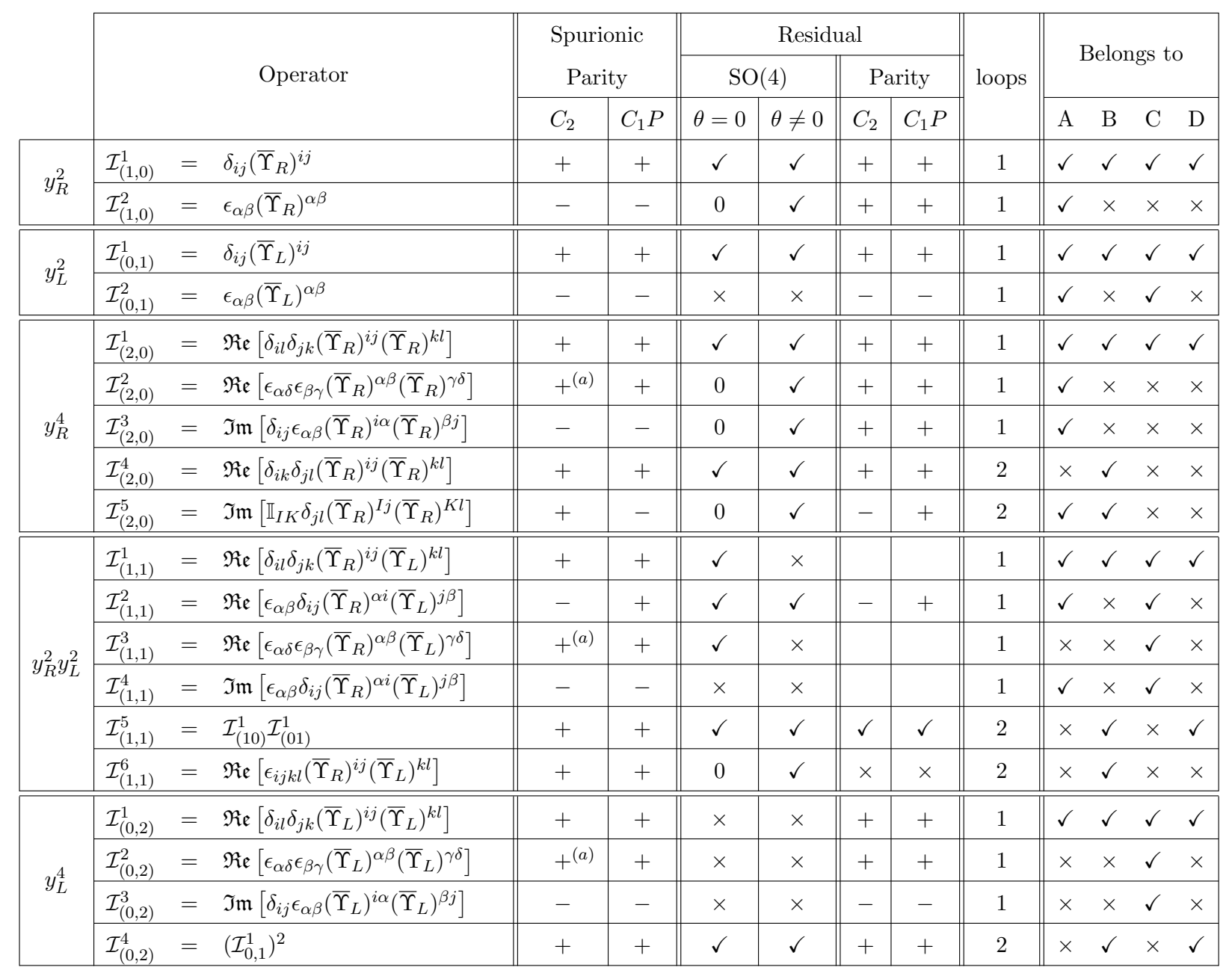

Table 2: The independent invariants that contribute to the Higgs potential, up to order $y_{L, R}^{4}$, in the case $\mathbf{r}_{Q, T}=\mathbf{6}$. For each operator, the first two columns contain the spurionic $C_{2}$ and $C_{1} P$ parities, the third and fourth ones indicate whether it will respect the $\mathrm{SO}(4)$ symmetry after the spurions acquire $\mathrm{VEV}$, while the following two show the $C_{2}$ and $C_{1} P$ parities of the generated potential. Whether the operator can be generated at one or two loops is written in the seventh column. The last columns indicate which operators should be used in a given setup; A: no constraints, B: $C_{2}$ in the strong sector, C: $C_{2}$ in the fermion coupling, and D: $C_{2}$ both in the strong sector and the fermion coupling. To order the operators we have given priority to 1 loop against 2 loops, and further assumed $g_{\rho}>y_{R}>y_{L}$. The shape of the potential is not affected by this choice, only the Naive Dimensional Analysis (NDA) associated to the various coefficients would be modified. $(a)$ : the intrisic $C_{2}$ is positive because it is the product of two $C_{2}$ odd contributions. In case of a $C_{2}$ symmetric strong sector, these operators would come at two loops. 
for our purposes, and are shown in table 3. The number of independent invariants is again obtained by counting, given the decomposition in eq. (50), the $\mathrm{SO}(4) \times \mathrm{SO}(2)$ singlets one can form with two $\mathbf{2 0}^{\prime}$ s. There are 6 of them, one of which however should be removed given that it corresponds to the trivial $\mathrm{SO}(6)$ invariant which does not contribute to the potential. Finally, the $y_{R}$ spurion will not contribute to the potential because the coupling of $t_{R}$ with an $\mathrm{SO}(6)$ singlet does not break the NGB symmetry. As already mentioned, $t_{R}$ could even be a completely composite state, corresponding to $y_{R} \rightarrow g_{\rho}$.

The results are similar to the ones obtained in the case of the 6 : at the $y^{2}$ order imposing any one of the discrete symmetries automatically implies the other and also $\mathrm{SO}(4)$ invariance. Moreover, $C_{1} P \cdot C_{2}$ is an accidental symmetry of the potential. Unlike the case of two $\mathbf{6}$, spurionic and residual symmetries coincide because we chose to restrict to a spurion $y_{L}$ that preserves $C_{1} P$ and $C_{2}$.

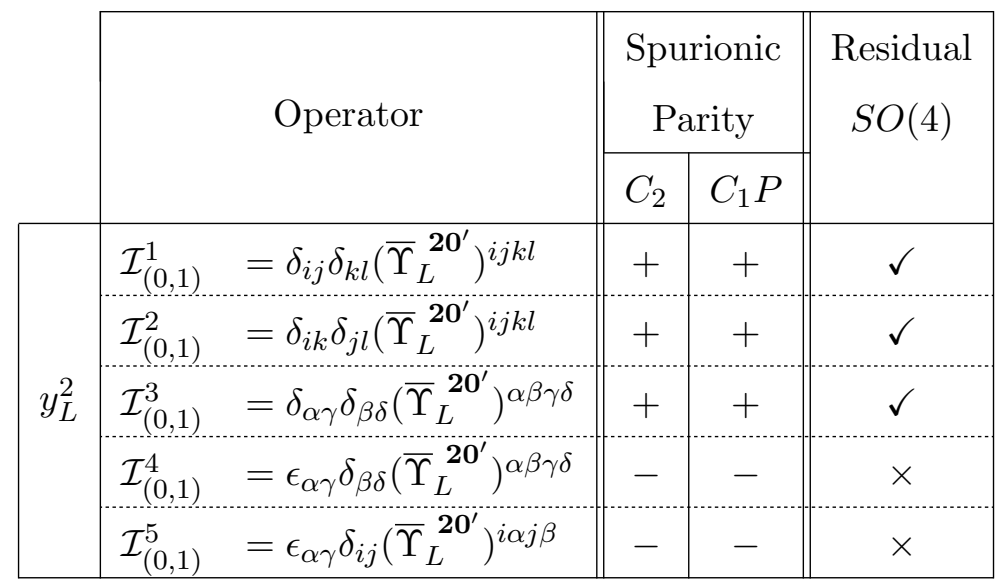

Table 3: The independent invariants that contribute to the Higgs potential, up to order $y_{L, R}^{2}$ for $\left\{\mathbf{r}_{Q}, \mathbf{r}_{T}\right\}=\left\{\mathbf{2 0}^{\prime}, \mathbf{1}\right\}$. For each operator, the first two columns contain its spurionic $C_{2}$ and $C_{1} P$ parities, the third one indicates whether it will respect the $\mathrm{SO}(4)$ symmetry after the spurions will have taken VEV.

\section{Gauge Contributions:}

Let us now discuss the gauge contributions to the potential, where few modifications of the above procedure will be needed. The starting point are now the couplings of the elementary $\mathrm{SU}(2)_{L} \times \mathrm{U}(1)_{Y}$ gauge fields $(W$ and $B)$ to the strong sector, given by

$$
\mathcal{L}_{\text {gauge }}=-W_{\mu \bar{a}}\left(g^{\bar{a}}\right)^{J I} J_{I J}^{\mu}-B_{\mu}\left(g^{\prime}\right)^{J I} J_{I J}^{\mu}-B_{\mu} g_{X}^{\prime} J_{X}^{\mu},
$$

where $J_{X}^{\mu}$ denotes the $\mathrm{U}(1)_{X}$ current while $J_{I J}^{\mu}$ is defined, in terms of the $\mathrm{SO}(6)$ currents $J_{A}^{\mu}$, by

$$
J_{I J}^{\mu} \equiv J_{A}^{\mu} T_{I J}^{A} .
$$

The Lagrangian in eq. (52) has precisely the same structure of eq. (35); it describes the coupling, due to the partial gauging of the strong sector's global group, of the elementary gauge fields to the global currents. These couplings, i.e. VEVs of the spurions $g$ and $g^{\prime}$ in eq. (52), are determined by identifying the $\mathrm{SU}(2)_{L}$ 
SM group factor with the $\mathrm{SU}(2)_{L}$ (in the notation of eq. (32) subgroup of $\mathrm{SO}(6)$ and hypercharge with $T_{R}^{3}+X$. They are given by

$$
\left(g^{\bar{a}}\right)^{I J}=g\left(T_{L}^{\bar{a}}\right)^{I J}, \quad\left(g^{\prime}\right)^{I J}=g^{\prime}\left(T_{R}^{3}\right)^{I J}, \quad g_{X}^{\prime}=g^{\prime} .
$$

where $g$ and $g^{\prime}$ are the $\mathrm{SU}(2)_{L}$ and $\mathrm{U}(1)_{Y}$ gauge couplings.

The $g$-spurion $\left(g^{\bar{a}}\right)^{I J}$ has, on top of the antisymmetric $[I, J]$ pair of $\mathrm{SO}(6)$ indices, an extra $\bar{a}=1,2,3$ index in the adjoint of the elementary $\mathrm{SU}(2)_{L}^{\mathrm{el}}$. One can easily see that invariants can be formed by either contracting the $g$-spurion with itself or with at least two powers of the $y_{L}$-spurion. The second possibility, corresponds, however, to higher-order terms. We thus conclude that, at leading order, the $g$-spurion can only enter the potential through the combination

$$
\left(\Gamma_{g}\right)^{I J K L} \equiv\left(g_{\bar{a}}\right)^{I J}\left(g^{\bar{a}}\right)^{K L},
$$

which is obviously antisymmetric in the $[I, J]$ and $[K, L]$ indices and symmetric under the simultaneous exchange of the $[I, J]$ and $[K, L]$ pairs. For what concerns $\left(g^{\prime}\right)^{I J}$ and $g_{X}^{\prime}$, because of the symmetry $B_{\mu} \rightarrow-B_{\mu}, g^{\prime} \rightarrow-g^{\prime}$, they can only enter via two combinations

$$
\left(\Gamma_{g^{\prime}}^{+}\right)^{I J K L} \equiv\left(g^{\prime}\right)^{I J}\left(g^{\prime}\right)^{K L}, \quad\left(\Gamma_{g^{\prime}}^{-}\right)^{I J} \equiv\left(g^{\prime}\right)^{I J} g_{X}^{\prime},
$$

having ignored the $\left(g_{X}^{\prime}\right)^{2}$ term that, being an $\mathrm{SO}(6)$ singlet will not contribute to the potential.

\begin{tabular}{|c|c|c|c|c|c|}
\hline & & \multirow[t]{2}{*}{ Operator } & \multicolumn{2}{|c|}{$\begin{array}{c}\text { Spurionic } \\
\text { Parity }\end{array}$} & \multirow[t]{2}{*}{$\begin{array}{l}\text { Residual } \\
S O(4)\end{array}$} \\
\hline & & & $C_{2}$ & $C_{1} P$ & \\
\hline \multirow{3}{*}{$g^{2}$} & $\mathcal{I}_{g}^{1}$ & $=\delta_{i k} \delta_{j l}\left(\bar{\Gamma}_{g}\right)^{i j k l}$ & + & + & $\checkmark$ \\
\hline & $\mathcal{I}_{g}^{2}$ & $=\delta_{\alpha \gamma} \delta_{\beta \delta}\left(\bar{\Gamma}_{g}\right)^{\alpha \beta \gamma \delta}$ & + & + & $\checkmark$ \\
\hline & $\mathcal{I}_{g}^{3}$ & $=\epsilon_{i j k l}\left(\bar{\Gamma}_{g}\right)^{i j k l}$ & + & + & $\checkmark$ \\
\hline \multirow{4}{*}{$g^{\prime 2}$} & $\mathcal{I}_{g^{\prime}}^{1}$ & $=\delta_{i k} \delta_{j l}\left(\bar{\Gamma}_{g^{\prime}}^{+}\right)^{i j k l}$ & + & + & $x$ \\
\hline & $\mathcal{I}_{g^{\prime}}^{2}$ & $=\delta_{\alpha \gamma} \delta_{\beta \delta}\left(\bar{\Gamma}_{g^{\prime}}^{+}\right)^{\alpha \beta \gamma \delta}$ & + & + & $\times$ \\
\hline & $\mathcal{I}_{g^{\prime}}^{3}$ & $=\epsilon_{i j k l}\left(\bar{\Gamma}_{g^{\prime}}^{+}\right)^{i j k l}$ & + & + & $\checkmark$ \\
\hline & $\mathcal{I}_{g^{\prime}}^{2}$ & $=\epsilon_{\alpha \beta}\left(\bar{\Gamma}_{g^{\prime}}^{-}\right)^{\alpha \beta}$ & - & - & $\times$ \\
\hline
\end{tabular}

Starting from the building blocks in eqs. (54 55), we can classify the possible contributions to the potential in terms of the $\mathrm{SO}(4) \times \mathrm{SO}(2)$ invariants that can be built out of $\Gamma_{g}$ and $\Gamma_{g^{\prime}}^{ \pm}$. At the leading

Table 4: Gauge contributions to the potential, constructed with the dressed spurions $\bar{\Gamma}_{g}, \bar{\Gamma}_{g^{\prime}}^{+}$, and $\bar{\Gamma}_{g^{\prime}}^{-}$. For each operator, the first two columns contain the intrinsic $C_{2}$ and $C_{1} P$ parities, and the third one indicates whether it will respect the $\mathrm{SO}(4)$ symmetry after the spurions have acquires VEVs. 
order, it is very simple to count the invariants, if one remembers that each $[I, J]$ and $[K, L]$ pair actually forms a single index in the adjoint, and that the adjoint decomposes as

$$
15=(6,1) \oplus(4,2) \oplus(1,1)
$$

under $\mathrm{SO}(4) \times \mathrm{SO}(2)$. Out of $\Gamma_{g}$, which is the product of two 15, 4 invariants can be formed ${ }^{13}$ one of which is however trivial, being associated to the $\mathrm{SO}(6)$ invariant. The same applies to $\Gamma_{g^{\prime}}^{+}$, while $\Gamma_{g^{\prime}}^{-}$only leads to one invariant, associated to the unique singlet in the decomposition of the $\mathbf{1 5}$. We are therefore left with 7 invariants, that are listed in table 4 together with their $C_{1} P$ and $C_{2}$ parities.

Concerning $C_{1} P$, few more comments are needed. We defined $C_{1} P$ to act as the standard $C P$ conjugation in the elementary sector; it therefore acts on the gauge fields as

$$
W_{\bar{a}} \rightarrow-(-)^{\delta_{\bar{a}}^{2}} W_{\bar{a}}^{(P)}, \quad B \rightarrow-B^{(P)},
$$

where the " $(P)$ " superscript denote the action of ordinary parity. On the strong sector, $C_{1} P$ acts again as ordinary $C P$, but convoluted with the $\mathcal{C}_{1} \mathrm{SO}(6)$ rotation defined in eq. (34). Under ordinary $C P$ each representation goes into its conjugate, so that the strong sector's currents transform as 14

$$
J_{I J} \rightarrow-\left(J_{I J}^{(P)}\right)^{*}=J_{I J}^{(P)}, \quad J_{X} \rightarrow-\left(J_{X}^{(P)}\right)^{*}=-J_{X}^{(P)} .
$$

Convoluting the above equation with $\mathcal{C}_{1}$ and making use of eq. (57), we immediately find that the spurions transform as

$$
\begin{aligned}
& \left(g^{\bar{a}}\right)^{I J} \rightarrow-(-)^{\delta_{\bar{a}}^{2}}\left(\mathcal{C}_{1}^{\mathbf{6}}\right)^{I}{ }_{K}\left(\mathcal{C}_{1}^{\mathbf{6}}\right)^{J}{ }_{L}\left(g^{\bar{a}}\right)^{K L}, \\
& \left(g^{\prime}\right)^{I J} \rightarrow-\left(\mathcal{C}_{1}^{\mathbf{6}}\right)^{I}{ }_{K}\left(\mathcal{C}_{1}^{\mathbf{6}}\right)^{J}{ }_{L}\left(g^{\prime}\right)^{K L}, \\
& g_{X}^{\prime} \rightarrow g_{X}^{\prime} .
\end{aligned}
$$

It is straightforward at this point to check that the spurion's VEVs in eq. (53) are invariant, meaning that the gauging of the SM group does not break $C_{1} P$ (provided that it was present as a symmetry of the strong sector). From the above equation one can also derive the $C_{1} P$ action on $\Gamma_{g}$ and on $\Gamma_{g^{\prime}}^{ \pm}$Up to the $\mathcal{C}_{1}$ rotation, we find that $\Gamma_{g}$ and $\Gamma_{g^{\prime}}^{+}$are even while $\Gamma_{g^{\prime}}^{-}$is odd; this explains the results of table 4

\subsection{2 $\quad C_{2}$ Invariant Models}

Armed with the technical tools of the previous section, we now describe some specific composite-Higgs scenarios, based on the $\mathrm{SO}(6) \rightarrow \mathrm{SO}(4) \times \mathrm{SO}(2)$ symmetry breaking pattern. In this section we will focus on the case in which the strongly interacting sector also possesses the additional $C_{2}$ symmetry, while the

\footnotetext{
${ }^{13}$ One should not forget, to perform the correct counting, that the $\mathbf{6}$ is reducible because it coincides with the adjoint $(\mathbf{3}, \mathbf{1}) \oplus(\mathbf{1}, \mathbf{3})$ in the $\mathrm{SU}(2)_{L} \times \mathrm{SU}(2)_{R}$ notation. Also, one of the two invariants one could form with two $(\mathbf{4}, \mathbf{2})$ actually vanishes because it is antisymmetric.

${ }^{14}$ The resulting signs simply follow from the fact that the generators of $\mathrm{SO}(6)$ are purely imaginary, while that of $\mathrm{U}(1)_{X}$ is real.
} 
case of $C_{1} P$ invariance will be described in the following one. Also, we restrict to the case of $\mathbf{r}_{Q, T}=\mathbf{6}$, in which left- and right-handed elementary fermions couple to composite operators in the $\mathbf{6}$; however the choice of $\left\{\mathbf{r}_{Q}, \mathbf{r}_{T}\right\}=\left\{\mathbf{2 0}^{\prime}, \mathbf{1}\right\}$ might equally well be considered. Notice that $C_{2}$-invariance of the strong sector does not imply that this is an exact symmetry, given that it might be broken explicitly by the coupling of elementary fermions or spontaneously by the VEV of the second Higgs $\Phi^{\hat{2}}$; both possibilities will be considered in what follows.

Introducing flavor indices in eq. (43), $f=u, c, t$, we have for the up sector,

$$
\begin{aligned}
\left(\left(y_{L}\right)_{f}^{\mathrm{u} \bar{\alpha}}\right)^{I} & =\frac{\left(y_{L}\right)_{f}^{\mathrm{u}}}{\sqrt{2}}\left\{\left(\vec{v}^{\overline{1}}, 0,0\right),\left(\vec{v}^{\overline{2}}, 0,0\right)\right\}, \\
\left(\left(y_{R}\right)_{f}^{\mathrm{u}}\right)^{I} & =\left(y_{R}\right)_{f}^{\mathrm{u}}\left(0,0,0,0, \cos \theta_{f}^{\mathrm{u}}, i e^{i \phi_{f}^{\mathrm{u}}} \sin \theta_{f}^{\mathrm{u}}\right),
\end{aligned}
$$

where $\left(y_{L}\right)_{f}^{\mathrm{u}}$ and $\left(y_{R}\right)_{f}^{\mathrm{u}}$ have been made real by, respectively, a $\mathrm{U}(1)_{L}^{\mathrm{el}}$ and $\mathrm{U}(1)_{R}^{\mathrm{el}}$ flavor-dependent rotation of the elementary fields. The vectors $\vec{v}^{\overline{1}, 2}$ are defined in eq. (44). By an $\mathrm{SO}(2)$ rotation in the strong sector group one can also eliminate, as we did in eq. (46), the phase $\phi_{t}^{\mathrm{u}}$ associated to the top quark, while the others remain physical. With more than one family, therefore, $C_{1} P \cdot C_{2}$ is not any longer automatically preserved (provided it was a symmetry of the strong sector to start with) by the up-type couplings.

The discussion is easily extended to the down-type Yukawa coupling. If, as we assume, the right-handed down quarks are coupled to a singlet of custodial symmetry, the Yukawas can be generated by coupling the left-handed SM doublet to a second operator in the $(\mathbf{2}, \mathbf{2})_{-\mathbf{1} / \mathbf{3}}$ representation of $\mathrm{SU}(2)_{L} \times \mathrm{SU}(2)_{R} \times \mathrm{U}(1)_{X}$. This can be realized with fermionic operators, $\mathcal{O}^{\prime}{ }_{Q_{Q^{\prime}}}$, again in the 6 representation, but with $X=-1 / 3$ charge:

$$
\mathcal{L}_{\text {mix }}=\left(\bar{q}_{L}\right)_{\bar{\alpha}}\left(y_{L}{ }^{\bar{\alpha}}\right)^{I_{Q^{\prime}}} \mathcal{O}^{\prime}{ }_{Q_{Q^{\prime}}}+\left(\bar{b}_{R}\right)\left(y_{R}\right)^{I_{B}} \mathcal{O}_{I_{B}}+\text { h.c. },
$$

where the flavor indices are understood. The embedding of the SM quarks in the representations above is given by

$$
\begin{aligned}
\left(\left(y_{L}\right)_{f}^{\mathrm{d}^{\bar{\alpha}}}\right)^{I} & =\frac{\left(y_{L}\right)_{f}^{\mathrm{d}}}{\sqrt{2}}\left\{\left(\left(\vec{v}^{\overline{2}}\right)^{*}, 0,0\right),\left(\left(\vec{v}^{\overline{1}}\right)^{*}, 0,0\right)\right\}, \\
\left(\left(y_{R}\right)_{f}^{\mathrm{d}}\right)^{I} & =\left(y_{R}\right)_{f}^{\mathrm{d}}\left(0,0,0,0, \cos \theta_{f}^{\mathrm{d}}, i e^{i \phi_{f}^{\mathrm{d}}} \sin \theta_{f}^{\mathrm{d}}\right),
\end{aligned}
$$

where $f=d, s, b$, and $\left(y_{R}\right)_{f}^{\mathrm{d}}$ has been made real by a $\mathrm{U}(1)_{R}^{\mathrm{el}}$ elementary rotation of the $d_{R}^{f}$ quarks. Of the remaining phases, one could be eliminated by a $\mathrm{U}(1)_{X}$ elementary rotation while all the others are physical. We stress that, as discussed in section 2.3 , a generic choice with flavor dependent $\theta_{f}^{\mathrm{d}}, \theta_{f}^{\mathrm{u}}, \phi_{f}^{\mathrm{d}}, \phi_{f}^{\mathrm{u}}$ would lead to Higgs-mediated FCNC.

The only $C_{2}$-invariant choice of the couplings is $\theta_{f}^{\mathrm{u}, \mathrm{d}}=0$, while, by taking $\theta_{f}^{\mathrm{u}}=0$ and $\theta_{f}^{\mathrm{d}}=\pi / 2$, we preserve $C_{I} \cdot C_{2}$, where $C_{I}$ is the isospin parity defined in section 2.3 under which the $d_{R}^{f}$ elementary quarks change sign 15 . Similarly, one could also introduce the lepton couplings and show that all the different scenarios of table 2.3 can be implemented by suitable choices of the mixing angles. In each of these

\footnotetext{
${ }^{15}$ Together with $\theta_{f}^{\mathrm{d}}=\pi / 2$ one can also take, by field redefinitions, $\phi_{f}^{\mathrm{d}}=0$.
} 
scenarios, as discussed in section 2.3, large Higgs-mediated FCNC are avoided. In the present framework, however, the type-I scenario results more natural than the others because it is the only one that does not require the second Higgs to take a VEV in order for the masses to be generated after EWSB. As we will see, the second Higgs acquiring (a not too large) VEV requires a certain additional fine-tuning.

Having introduced the general framework, let us now discuss its possible vacuum structures, which are determined by the form of the Higgs potential. The latter is insensitive to the light fermion couplings, so that the discussion which follows is independent of the choices of the mixing angles and phases, apart of course from those of the top quark in eqs. 43 46). We denote, as in the previous section, $\theta_{t}^{\mathrm{u}}$ as $\theta$. Having assumed $C_{2}$-invariance of the strong sector, the allowed contributions to the potential are the intrinsic $C_{2}$ even operators listed in tables 2 and 4 . As already explained, no accidental symmetry is expected in the general case in which $\theta \neq 0$ in eq. (46) as both $C_{1} P$ and $\mathrm{SO}(4)$ are broken by the top quark proto-Yukawas. But, by choosing the top-quark coupling to respect $C_{2}$ (i.e., $\theta=0$ ), the potential becomes separately invariant under $C_{1} P$ and $C_{2}$. Moreover, at the leading $y^{2}$ order one obtains accidental $\mathrm{SO}(4)$. Besides these important symmetry considerations, we can make a more concrete use of the results in tables 2 and 4. Expanding in powers of $\Phi_{\widehat{1}, \hat{2}} / f$, each invariant will give a specific contribution to the parameters of the general renormalizable $2 \mathrm{HDM}$ potential

$$
\begin{aligned}
& V\left(\Phi_{\widehat{1}}, \Phi_{\widehat{2}}\right)=\frac{1}{2} m_{11}^{2} \operatorname{Tr}\left[\Phi_{\widehat{1}}^{\dagger} \Phi_{\widehat{1}}\right]+\frac{1}{2} m_{22}^{2} \operatorname{Tr}\left[\Phi_{\widehat{2}}^{\dagger} \Phi_{\widehat{\mathbf{2}}}\right]+\frac{1}{2} \operatorname{Tr}\left[\Phi_{\widehat{1}}^{\dagger} \Phi_{\widehat{\mathbf{2}}}\left(m_{12}^{2}+i \tilde{m}_{12}^{2} \sigma_{3}\right)\right] \\
& +\frac{1}{4} \lambda_{1} \operatorname{Tr}^{2}\left[\Phi_{\widehat{1}}^{\dagger} \Phi_{\widehat{1}}\right]+\frac{1}{4} \lambda_{2} \operatorname{Tr}^{2}\left[\Phi_{\widehat{2}}^{\dagger} \Phi_{\widehat{2}}\right]+\frac{1}{4} \lambda_{3} \operatorname{Tr}\left[\Phi_{\widehat{1}}^{\dagger} \Phi_{\widehat{1}}\right] \operatorname{Tr}\left[\Phi_{\widehat{2}}^{\dagger} \Phi_{\widehat{2}}\right] \\
& +\frac{1}{4} \lambda_{4} \operatorname{Tr}^{2}\left[\Phi_{\widehat{1}}^{\dagger} \Phi_{\hat{2}}\right]+\frac{1}{4} \tilde{\lambda}_{4} \operatorname{Tr}^{2}\left[\Phi_{\widehat{1}}^{\dagger} \Phi_{\widehat{2}} \sigma_{3}\right]+i \frac{1}{4} \lambda_{5} \operatorname{Tr}\left[\Phi_{\widehat{1}}^{\dagger} \Phi_{\widehat{2}}\right] \operatorname{Tr}\left[\Phi_{\widehat{1}}^{\dagger} \Phi_{\widehat{2}} \sigma_{3}\right] \\
& +\frac{1}{4} \operatorname{Tr}\left[\Phi_{\widehat{1}}^{\dagger} \Phi_{\widehat{1}}\right] \operatorname{Tr}\left[\Phi_{\widehat{1}}^{\dagger} \Phi_{\widehat{2}}\left(\lambda_{6}+i \tilde{\lambda}_{6} \sigma_{3}\right)\right]+\frac{1}{4} \operatorname{Tr}\left[\Phi_{\widehat{2}}^{\dagger} \Phi_{\widehat{2}}\right] \operatorname{Tr}\left[\Phi_{\widehat{1}}^{\dagger} \Phi_{\widehat{2}}\left(\lambda_{7}+i \tilde{\lambda}_{7} \sigma_{3}\right)\right] .
\end{aligned}
$$

These contributions are summarized in tables 5 and 6, it is understood that each term is proportional to the corresponding coefficient $c_{\delta}^{\left(n_{R}, n_{L}\right)}$ appearing in eq. 37. From the tables we see that the coefficient $\lambda_{5}$ is not generated at the order we are working, since it breaks $\mathrm{SO}(4)$ while being $C_{1} P$ odd and $C_{2}$ even. The first contribution to $\lambda_{5}$ comes at order $y_{L}^{4} y_{R}^{2}$. For $\theta=0, m_{12}^{2}, \tilde{m}_{12}^{2}, \lambda_{6,7}$ and $\tilde{\lambda}_{6,7}$ also vanish due to the separate $C_{2}$ and accidental $C_{1} P$ symmetries.

\section{Composite Inert Higgs}

Let us now consider the case in which, by choosing $\theta_{f}^{\mathrm{u}, \mathrm{d}}=0, C_{2}$ is preserved by all the fermion couplings. Provided the potential allows $\Phi^{\widehat{2}}$ to have zero VEV, we obtain a composite realization of the inert Higgs scenario [4] in which the $C_{2}$ symmetry is completely unbroken. In this case, the lightest component of the $C_{2}$-odd Higgs doublet becomes absolutely stable, providing a potential dark matter candidate.

Treating the coefficients $c_{\delta}^{\left(n_{R}, n_{L}\right)}$ as $O(1)$ free parameters, and assuming that they can take both signs, a "large" region of the parameter space is easily identified where EWSB occurs with the second Higgs not taking a VEV, so that $C_{2}$ is unbroken. However, we need something more for a potentially realistic 


\begin{tabular}{|c|c|c|c|c|c|c|c|c|c|c|}
\hline operator & $\mathcal{I}_{(0,1)}^{1}$ & $\mathcal{I}_{(1,0)}^{1}$ & $\mathcal{I}_{(2,0)}^{1}$ & $\mathcal{I}_{(2,0)}^{4}$ & $\mathcal{I}_{(2,0)}^{5}$ & $\mathcal{I}_{(1,1)}^{1}$ & $\mathcal{I}_{(1,1)}^{5}$ & $\mathcal{I}_{(1,1)}^{6}$ & $\mathcal{I}_{(0,2)}^{1}$ & $\mathcal{I}_{(0,2)}^{4}$ \\
\hline$\frac{1}{16 \pi^{2}} \times$ & $-\frac{y_{L}^{2} g_{\rho}^{2}}{2}$ & $y_{R}^{2} g_{\rho}^{2}$ & $\frac{y_{R}^{4}}{4}$ & $\frac{y_{R}^{4}}{4}\left(\frac{g_{\rho}}{4 \pi}\right)^{2}$ & $\frac{y_{R}^{4}}{4}\left(\frac{g_{\rho}}{4 \pi}\right)^{2}$ & $\frac{y_{R}^{2} y_{L}^{2}}{4}$ & $y_{R}^{2} y_{L}^{2}\left(\frac{g_{\rho}}{4 \pi}\right)^{2}$ & $-y_{R}^{2} y_{L}^{2}\left(\frac{g_{\rho}}{4 \pi}\right)^{2}$ & $-\frac{y_{L}^{4}}{2}$ & $-y_{L}^{4}\left(\frac{g_{\rho}}{4 \pi}\right)^{2}$ \\
\hline$m_{11}^{2} / f^{2}$ & 1 & $\cos ^{2} \theta$ & 0 & 0 & 0 & $\cos ^{2} \theta$ & $\cos ^{2} \theta$ & 0 & 1 & 1 \\
\hline$m_{22}^{2} / f^{2}$ & 1 & $\sin ^{2} \theta$ & 0 & 0 & 0 & $\sin ^{2} \theta$ & $\sin ^{2} \theta$ & 0 & 1 & 1 \\
\hline$m_{12}^{2} / f^{2}$ & 0 & 0 & 0 & 0 & $\sin 4 \theta$ & 0 & 0 & 0 & 0 & 0 \\
\hline$\tilde{m}_{12}^{2} / f^{2}$ & 0 & 0 & 0 & 0 & 0 & $-\sin 2 \theta$ & 0 & $\frac{1}{2} \sin 2 \theta$ & 0 & 0 \\
\hline$\lambda_{1}$ & $-\frac{1}{3}$ & $-\frac{1}{3} \cos ^{2} \theta$ & $2 \cos ^{4} \theta$ & $2 \cos ^{4} \theta$ & 0 & $-\frac{4}{3} \cos ^{2} \theta$ & $-\frac{7}{12} \cos ^{2} \theta$ & 0 & $-\frac{7}{12}$ & $-\frac{11}{24}$ \\
\hline$\lambda_{2}$ & $-\frac{1}{3}$ & $-\frac{1}{3} \sin ^{2} \theta$ & $2 \sin ^{4} \theta$ & $2 \sin ^{4} \theta$ & 0 & $-\frac{4}{3} \sin ^{2} \theta$ & $-\frac{7}{12} \sin ^{2} \theta$ & 0 & $-\frac{7}{12}$ & $-\frac{11}{24}$ \\
\hline$\lambda_{3}$ & 0 & 0 & $\sin ^{2} \theta$ & $-\sin ^{2} \theta$ & 0 & 0 & $-\frac{1}{4}$ & 0 & 0 & $-\frac{1}{4}$ \\
\hline$\lambda_{4}$ & $-\frac{2}{3}$ & $-\frac{1}{3}$ & 0 & $2 \sin ^{2} 2 \theta$ & 0 & $-\frac{4}{3}$ & $-\frac{1}{3}$ & 0 & $-\frac{7}{6}$ & $-\frac{2}{3}$ \\
\hline$\tilde{\lambda}_{4}$ & 0 & 0 & 0 & 0 & 0 & 0 & 0 & 0 & $\frac{1}{2}$ & 0 \\
\hline$\lambda_{5}$ & 0 & 0 & 0 & 0 & 0 & 0 & 0 & 0 & 0 & 0 \\
\hline$\lambda_{6}$ & 0 & 0 & 0 & 0 & $-\frac{1}{3} \sin 4 \theta$ & 0 & 0 & 0 & 0 & 0 \\
\hline$\tilde{\lambda}_{6}$ & 0 & 0 & 0 & 0 & 0 & $\frac{2}{3} \sin 2 \theta$ & 0 & $-\frac{1}{12} \sin 2 \theta$ & 0 & 0 \\
\hline$\lambda_{7}$ & 0 & 0 & 0 & 0 & $-\frac{1}{3} \sin 4 \theta$ & 0 & 0 & 0 & 0 & 0 \\
\hline$\tilde{\lambda}_{7}$ & 0 & 0 & 0 & 0 & 0 & $\frac{2}{3} \sin 2 \theta$ & 0 & $-\frac{1}{12} \sin 2 \theta$ & 0 & 0 \\
\hline
\end{tabular}

Table 5: Contribution to the parameters of the general $2 \mathrm{HDM}$ potential eq. 63 from fermions in the 6. The individual contributions of the $\mathrm{SO}(6) / \mathrm{SO}(4) \times \mathrm{SO}(2)$ operators of table 2 are shown. The first line indicates the power-counting estimate of the pre-factor.

composite inert-Higgs model. As we explained before, satisfying the EWPT requires the VEV of the first Higgs to satisfy eq. (26), that, unless $g_{\rho}$ is maximally large, implies $\xi \ll 1$. This can only be achieved by advocating a cancellation among the different contributions to the mass-parameter $m_{11}^{2}$ in eq. (63), which must become negative and smaller than what was expected by the NDA counting of eq. (37). We see from table 5 that at the leading $y^{2}$-order the two separated contributions to $m_{11}^{2}$ can be canceled one with each other. This cancellation, however, also reduces the quartic $\lambda_{1}$, making it useless for reducing the Higgs VEV, which remains of order $f$. This accident, which also occurs in the models of refs. [2, 6], renders the $O\left(y^{2}\right)$ potential not tunable, and is the very same reason why we have been obliged to retain the higher order $\left(y^{4}, g^{2}\right.$ and $\left.g^{\prime 2}\right)$ contributions.

If the higher-order terms are taken into account, the tuning becomes possible; we must demand the leading $y^{2}$ contributions to $m_{11}^{2}$ to be a factor $\xi$ smaller than the subleading one, those of order $y^{4}$ and $g^{2}$. In this case the quartic $\lambda_{1}$ is dominated by the higher-order contributions:

$$
\lambda_{1} \sim \frac{1}{16 \pi^{2}} \operatorname{Max}\left\{N_{c} y_{L}^{4}, N_{c} y_{R}^{4}, N_{c} y_{L}^{2} y_{R}^{2}, g^{2} g_{\rho}^{2}, g^{\prime 2} g_{\rho}^{2},\right\},
$$

where the $N_{c}=3$ color factor has been included in the estimates of the fermion's contributions. The 


\begin{tabular}{|c||c|c|c||c|c|c|}
\hline operator & $\mathcal{I}_{g}^{1}$ & $\mathcal{I}_{g}^{2}$ & $\mathcal{I}_{g}^{3}$ & $\mathcal{I}_{g^{\prime}}^{1}$ & $\mathcal{I}_{g^{\prime}}^{2}$ & $\mathcal{I}_{g^{\prime}}^{3}$ \\
\hline$\frac{g_{\rho}^{2}}{16 \pi^{2}} \times$ & $\frac{3}{4} g^{2}$ & $\frac{3}{2} g^{2}$ & $-\frac{1}{8} g^{2}$ & $\frac{1}{4} g^{\prime 2}$ & $\frac{1}{8} g^{\prime 2}$ & $-\frac{1}{2} g^{\prime 2}$ \\
\hdashline$m_{11}^{2} / f^{2}$ & 1 & 0 & 1 & 1 & 0 & 1 \\
$m_{22}^{2} / f^{2}$ & 1 & 0 & 1 & 1 & 0 & 1 \\
\hdashline$\lambda_{1}$ & $-\frac{1}{3}$ & 0 & $-\frac{1}{12}$ & $-\frac{1}{3}$ & 0 & $-\frac{1}{12}$ \\
$\lambda_{2}$ & $-\frac{1}{3}$ & 0 & $-\frac{1}{12}$ & $-\frac{1}{3}$ & 0 & $-\frac{1}{12}$ \\
$\lambda_{3}$ & $-\frac{1}{3}$ & 2 & $-\frac{1}{2}$ & 0 & 0 & $-\frac{1}{2}$ \\
$\lambda_{4}$ & $-\frac{1}{3}$ & -2 & $\frac{1}{3}$ & $-\frac{2}{3}$ & 0 & $\frac{1}{3}$ \\
$\tilde{\lambda}_{4}$ & 0 & 0 & 0 & 1 & 2 & 0 \\
\hline
\end{tabular}

Table 6: Contribution to the parameters of the general $2 \mathrm{HDM}$ potential eq. 63 from $\mathrm{SU}(2)_{L}$ and $\mathrm{U}(1)_{Y}$ gauge bosons. The individual contributions of the $\mathrm{SO}(6) / \mathrm{SO}(4) \times \mathrm{SO}(2)$ operators of table 4 are shown. The first line indicates the $\mathrm{NDA}$ pre-factor.

coefficients controlling the contributions of $y_{L}^{2}$ and $y_{R}^{2}$ to $m_{11}^{2}$ are plausibly expected to be comparable. Then a cancellation in $m_{11}^{2}$ would require $y_{L} \sim y_{R}$ which implies, given eq. (8), $y_{L} \sim y_{R} \sim \sqrt{Y_{t} g_{\rho}}$. We will assume those values for our estimates below. We stress that this choice does not create a tension with EWPT thanks to the accidental $P_{L R}$ discussed in section 2.4. Because $\lambda_{1}$ is given by eq. (64), we have that the lightest scalar in the spectrum is expected to be the $C_{2}$-even neutral scalar, $h$, which is contained in the first Higgs doublet $\Phi_{\hat{1}}$. The mass of $h$ can be estimated as

$$
m_{h}^{2} \sim \lambda_{1} v_{1}^{2} \sim(250 \mathrm{GeV})^{2}\left(\frac{3}{N}\right)
$$

where $N$ is defined in eq. (1). The potential of the second (inert) Higgs doublet $\Phi_{\widehat{2}}$ is dominated by the leading order $y^{2}$ contribution, which is basically fixed up to an $O(1)$ overall coefficient once a cancellation in $m_{11}^{2}$ is assumed

$$
V \simeq N_{c} \frac{g_{\rho} Y_{t}}{16 \pi^{2}} m_{\rho}^{2}\left(\operatorname{Tr}\left[\Phi_{\widehat{2}} \cdot \Phi_{\widehat{2}}\right]-\frac{1}{6 f^{2}} \operatorname{Tr}^{2}\left[\Phi_{\widehat{2}} \cdot \Phi_{\widehat{2}}\right]-\frac{1}{6 f^{2}} \operatorname{Tr}^{2}\left[\Phi_{\widehat{1}} \cdot \Phi_{\widehat{\mathbf{2}}}\right]\right) .
$$

Decomposing $\Phi_{\widehat{2}}$ in its $\mathrm{SO}(3)_{c}$ triplet and singlet components, $H^{a}(a=1,2,3)$ and $H$ respectively, we see that the first term in the above equation gives a common contribution to the masses of all the components of order

$$
m_{H_{2}}^{2} \sim N_{c} \frac{g_{\rho}^{3} Y_{t}}{16 \pi^{2}} f^{2} \simeq(1.3 \mathrm{TeV})^{2}\left(\frac{3}{N}\right)^{\frac{3}{2}}\left(\frac{0.25}{\xi}\right)
$$


where we have used the relation $m_{\rho} \simeq g_{\rho} f$. The third term of eq. 66 induces a singlet-triplet splitting. Given that the overall sign of eq. 66 must be positive in order for $m_{H_{2}}^{2}$ to be positive, the sign of the splitting is fixed and the singlet $H$ is always lighter than the triplet $H^{a}$ :

$$
m_{H}^{2} \simeq\left(1-\frac{\xi}{3}\right) m_{H^{a}}^{2} .
$$

As discussed in the previous section and explicitly shown in eq. 66), the $y^{2}$ potential is $\mathrm{SO}(4)$-invariant so that the $\mathrm{SO}(3)_{c}$ breaking splittings among the charged and neutral triplet components, defined respectively as $H^{ \pm}=\left(H^{2} \pm i H^{1}\right) / \sqrt{2}$ and $A=H^{3}$, only come at order $g^{\prime 2}$ and $y^{4}$. These splittings can be respectively estimated as

$$
\begin{gathered}
\left|\frac{m_{H^{ \pm}}-m_{A}}{m_{H^{ \pm}}}\right|_{g^{\prime}} \sim \frac{g^{\prime 2} \xi}{g_{\rho} Y_{t}} \simeq 0.004 \sqrt{\frac{N}{3}}\left(\frac{\xi}{0.25}\right), \\
\left|\frac{m_{H^{ \pm}}-m_{A}}{m_{H^{ \pm}}}\right|_{y^{4}} \sim \frac{Y_{t} \xi}{g_{\rho}} \simeq 0.03 \sqrt{\frac{N}{3}}\left(\frac{\xi}{0.25}\right) .
\end{gathered}
$$

\section{Spontaneous $C_{2}$ Breaking}

Still assuming that $C_{2}$ is preserved by the couplings, we now consider the possibility that the second Higgs $\Phi^{\widehat{2}}$ also acquires a VEV. In this case $C_{2}$ is spontaneously broken, and the second Higgs multiplet is no longer inert. Also, a VEV of $\Phi^{\widehat{2}}$ is compulsory in order for the alternative scenarios (type-I, II, X and Y defined in section 2.3) to become viable. The discussion which follows applies to these scenarios as well.

If the VEV of $\Phi_{\widehat{2}}$ is non-zero, so breaking the discrete symmetry $\left(C_{2}\right.$ or $C_{I} \cdot C_{2}$, depending on the flavor embedding), large corrections to $\widehat{T}$ could be generated from the misalignment of the two VEVs, see section. 2.2. Avoiding these corrections was the very reason to advocate $C_{2}$, that was crucially assumed to be unbroken. Since we are now interested in choosing the parameters such that the vacuum is $C_{2}$-breaking, in this case the $C_{2}$ symmetry of the Lagrangian does not protect us anymore from large corrections to $\widehat{T}$. Fortunately this does not happen due to the accidental $C_{1} P$-invariance of the potential which is automatically present at the order we are working at. Because of $C_{1} P$, the two VEVs are aligned and large contributions to $\widehat{T}$ are avoided. We stress that it is only because of this accidental symmetry of the potential that the scenario of spontaneous $C_{2}$ breaking become phenomenologically viable in the present framework. The sub-leading effects that induce $\widehat{T}$ come from the breaking of $C_{1} P$ in the potential. The leading contribution comes at order $y_{L}^{4} y_{R}^{2}$ and gives rise to $\lambda_{5} \sim y_{L}^{4} y_{R}^{2} /\left(4 \pi g_{\rho}\right)^{2} \sim Y_{t}^{3} g_{\rho} / 16 \pi^{2}$. This generates a nonzero $\mathrm{VEV} v_{3}^{\widehat{2}}$ which can be estimated as

$$
v_{3}^{\widehat{2}} \sim \frac{\lambda_{5}\left(v_{4}^{\widehat{1}}\right)^{2}}{m_{22}^{2}} v_{4}^{\widehat{2}} \sim 0.05 \sqrt{\frac{N}{3}} v
$$

where we assumed a sizable spontaneous breaking of $C_{2}, v_{4}^{\widehat{1}} \sim v_{4}^{\widehat{2}} \sim v / \sqrt{2}$. The contributions to $\widehat{T}$ from this effect are under control

$$
\widehat{T}=\frac{\left(v_{4}^{\widehat{1}}\right)^{2}\left(v_{3}^{\widehat{2}}\right)^{2}}{f^{2} v^{2}} \sim 6 \cdot 10^{-4}\left(\frac{N}{3}\right)\left(\frac{\xi}{0.25}\right)
$$


We have explicitly checked that the free parameters of our potential naturally allow for the two Higgses to take (aligned, as we have seen) VEVs, but the request that both VEVs are smaller than $f$ clearly requires fine-tuning. The amount of fine-tuning is the square of that in the single-VEV case because both Higgs mass terms $m_{11}^{2}$ and $m_{22}^{2}$ need now to be reduced independently, such that $O\left(y^{2}\right)$ terms in the potential are comparable to $O\left(y^{4}\right)$ terms. Looking at tables 5 and 6 , we see that this requires that the coefficients of both operators arising at $O\left(y^{2}\right)$ be small, due to some (perhaps unappealing) peculiarity of the strong sector. In that case, the patterns in the Higgs spectrum described in the previous section for the inert Higgs scenario are not anymore present, and no sharp predictions can be made. We can estimate that all the masses will now be reduced by these tunings, and therefore all of them will be comparable and of the order of $m_{h}$, given in eq. (65).

\section{Explicit $C_{2}$ Breaking}

For $\theta_{f}^{\mathrm{u}, \mathrm{d}} \neq 0$ the $C_{2}$ and $C_{1} P$ symmetries are broken by the couplings of the SM fermions to the strong sector and, in principle, we are no longer protected against sizable contributions to FCNC processes or the $\widehat{T}$ parameter.

Nevertheless, as we mentioned above, flavor problems can be avoided if for some reason the protoYukawa matrices are aligned: $\theta_{f}^{\mathrm{u}, \mathrm{d}} \equiv \theta^{\mathrm{u}, \mathrm{d}}$ and $\phi_{f}^{\mathrm{u}, \mathrm{d}} \equiv \phi^{\mathrm{u}, \mathrm{d}}$. In this situation we have the composite version of type III 2HDM. The remarkable propery of this model is that the $O\left(y^{2}\right)$ potential is invariant under $C_{2}, C_{1} P$ and $\mathrm{SO}(4)$ custodial. As long as we are interested in vacua with a hierarchy $v \ll f$, the leading sources of breaking of those symmetries are given by the mass terms $\tilde{m}_{12}^{2}$ and $m_{12}^{2}$. The former respects only $C_{1} P \cdot C_{2}$ and arises at order $y_{L}^{2} y_{R}^{2} \sim Y_{t}^{2} g_{\rho}^{2}$ (see table 5). The latter breaks $C_{2}$ but preserves custodial and $C_{1} P$ and arises at order $g_{\rho}^{2} y_{R}^{4} / 16 \pi^{2} \sim Y_{t}^{2} g_{\rho}^{4} / 16 \pi^{2}$, and is thus normally further suppressed with respect to $\tilde{m}_{12}^{2}$. Assuming electroweak symmetry breaking is primarily triggered by the expectation value of $\left\langle\Phi_{\widehat{1}}\right\rangle=\left(0,0,0, v_{4}^{\widehat{1}}\right)$ we then have the following estimates for the entries in $\left\langle\Phi_{\widehat{2}}\right\rangle$

$$
\begin{aligned}
v_{3}^{\widehat{2}} & \simeq \frac{\tilde{m}_{12}^{2}}{m_{22}^{2}} v_{4}^{\widehat{1}} \sim \frac{Y_{t} \sin 2 \theta}{g_{\rho}} v_{4}^{\widehat{1}} \ll v \\
v_{4}^{\widehat{2}} & \simeq \frac{m_{12}^{2}}{m_{22}^{2}} v_{4}^{\widehat{1}} \sim \frac{Y_{t} g_{\rho} \sin 4 \theta}{16 \pi^{2}} v_{4}^{\widehat{1}} \ll v
\end{aligned}
$$

corresponding to a small breaking of $C_{2}, C_{1} P$ and $\mathrm{SO}(4)$. The resulting contribution to $\widehat{T}$ is given by

$$
\widehat{T}=\frac{\left(v_{4}^{\widehat{1}}\right)^{2}\left(v_{3}^{\widehat{2}}\right)^{2}}{f^{2} v^{2}} \sim \frac{\left(v_{4}^{\widehat{1}}\right)^{4}}{f^{2} v^{2}} \frac{Y_{t}^{2}}{g_{\rho}^{2}} \sin ^{2} 2 \theta \simeq 10^{-3}\left(\frac{\sin 2 \theta}{1 / 2}\right)^{2}\left(\frac{N}{3}\right)\left(\frac{\xi}{0.25}\right) .
$$

As one can see, the experimental constraint $\widehat{T} \lesssim 2 \times 10^{-3}$ can reasonably be satisfied. Interestingly, this contribution to $\widehat{T}$ is positive, which might even help to fit the EWPT.

In models with explicit breaking of $C_{2}$ the Higgs spectrum follows the estimates of the inert Higgs. The mixing of $h$ with $H(A)$ is small due to the approximate $C_{1} P \cdot C_{2}$ (custodial) symmetry. The only relevant 
phenomenological implication, as we will explore later, is that $\Phi_{\widehat{2}}$ couples now to fermions:

$$
i \tan \theta^{\mathrm{u}} \bar{q}_{L} \frac{M_{\mathrm{u}}}{v / \sqrt{2}} u_{R} \widetilde{H}_{\widehat{2}}+i e^{i \phi^{\mathrm{d}}} \tan \theta^{\mathrm{d}} \bar{q}_{L} \frac{M_{\mathrm{d}}}{v / \sqrt{2}} d_{R} H_{\widehat{\mathbf{2}}}+\text { h.c. },
$$

where $M_{\mathrm{u}, \mathrm{d}}$ are the fermion mass matrices.

\subsection{3 $\quad C_{1} P$ Invariant Models}

We now turn to models based on the $C_{1} P$ invariance of the strong sector. $C_{1} P$ and $C_{2}$ might also be imposed simultaneously with results similar to section 3.1 .2 , but we now want to consider the case where $C_{2}$ is maximally broken. To construct a model of this sort we cannot employ the setup of the previous section in which the quarks mix to composite operators with $\left\{\mathbf{r}_{Q}, \mathbf{r}_{T}\right\}=\{\mathbf{6}, \mathbf{6}\}$, because four Yukawa structures (two for the up-type and two for the down-type) would be present and, as discussed in section 2.3 , this would lead to large Higgs-mediated FCNC. In order to avoid the second set of Yukawas, forbidden in the previous section by the $C_{2}$ symmetry of the strong sector, we will use the $\left\{\mathbf{r}_{Q}, \mathbf{r}_{T}\right\}=\left\{\mathbf{2 0}^{\prime}, \mathbf{1}\right\}$ representations. A single invariant is now allowed by the $\mathrm{SO}(6)$ symmetry (see section 2.3). Then Higgs-mediated FCNC are avoided if one assumes the proto-Yukawa matrices have a flavor independent orientation (corresponding to a flavor independent embedding of the left-handed doublets into the $\mathbf{2 0}$ ).

Assuming the up-quark proto-Yukawas are $C_{1} P$ invariant, and using $\mathrm{SO}(2)$ rotations, we can in general write them in the form (see eq. (51))

$$
\left(\left(y_{L}\right)_{f}^{\mathrm{u} \bar{\alpha}}\right)^{I J}=\left(y_{L}\right)_{f}^{\mathrm{u}}\left\{\left(\begin{array}{c|c|c}
0_{4 \times 4} & \left(\vec{v}^{\overline{1}}\right)^{T} & 0_{4 \times 1} \\
\hline \vec{v}^{\overline{1}} & 0_{2 \times 2} \\
0_{1 \times 4} & 0_{2 \times 2}
\end{array}\right),\left(\begin{array}{c|cc}
0_{4 \times 4} & \left(\vec{v}^{\overline{2}}\right)^{T} & 0_{4 \times 1} \\
\hline \vec{v}^{\overline{2}} & 0_{2 \times 2} \\
0_{1 \times 4} & { }^{2}
\end{array}\right)\right\},
$$

which is found to accidentally respect $C_{2}$ as well.

In principle, since $C_{2}$ is not a symmetry of the strong sector, the fact that it is preserved by the coupling of elementary fermions should not imply any relevant consequence. Nevertheless, we find that some important terms generated by the strong sector will be accidentally $C_{2}$ invariant, and the model will, for this reason, resemble the Inert Higgs in several aspects, as we now discuss. One accidentally $C_{2}-$ invariant term is the (unique, as remarked above) up-type generalized Yukawa term constructed with the $\mathbf{2 0}^{\prime}$ and the singlet. Provided the second Higgs does not have a VEV, then all the interactions mediated by this term (i.e., remarkably, the ones involving the $t_{R}$ quark) will respect $C_{2}$. The down-type Yukawas are also generated by the coupling of the $q_{L}$ to another $\mathbf{2 0}^{\prime}$, with $X=-1 / 3$, while the $d_{R}$ couples to a $\mathbf{1}_{\mathbf{2} / \mathbf{3}}$. The right-handed proto-Yukawa $y_{R}$ is a singlet, while the most general $C_{1} P$ invariant $y_{L}$ proto-Yukawa for the downs reads

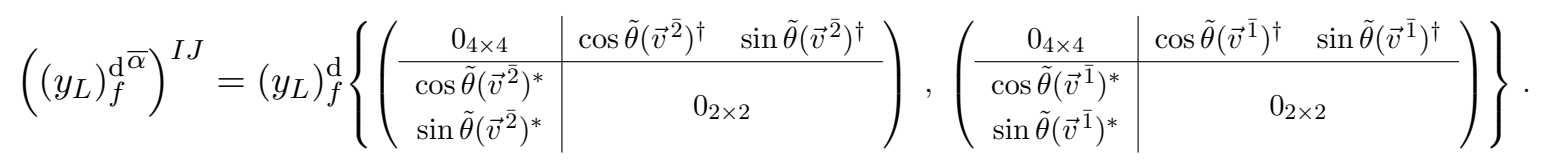

Notice that $\tilde{\theta}$ cannot be rotated away since $\mathrm{SO}(2)$ invariance has already been used to put the up protoYukawa into the form of eq. (76). Therefore the down-type proto-Yukawas do not respect the accidental $C_{2}$. 


\begin{tabular}{|c||c|c|c|}
\hline Operator & $\mathcal{I}_{(0,1)}^{1}$ & $\mathcal{I}_{(0,1)}^{2}$ & $\mathcal{I}_{(0,1)}^{3}$ \\
\hline$\frac{1}{16 \pi^{2}} \times$ & $y_{L}^{2} g_{\rho}^{2}$ & $-\frac{5}{2} y_{L}^{2} g_{\rho}^{2}$ & $y_{L}^{2} g_{\rho}^{2}$ \\
\hdashline$m_{11}^{2} / f^{2}$ & 1 & 1 & 1 \\
$m_{22}^{2} / f^{2}$ & 0 & 0 & $\frac{1}{2}$ \\
\hdashline$\lambda_{1}$ & $-\frac{4}{3}$ & $-\frac{11}{15}$ & $-\frac{4}{3}$ \\
$\lambda_{2}$ & 0 & 0 & $-\frac{1}{6}$ \\
$\lambda_{3}$ & 0 & $-\frac{1}{5}$ & $-\frac{1}{2}$ \\
$\lambda_{4}$ & $-\frac{4}{3}$ & $-\frac{8}{15}$ & -1 \\
$\tilde{\lambda}_{4}$ & 0 & 0 & 0 \\
\hline
\end{tabular}

Table 7: Contribution to the parameters of the general $2 \mathrm{HDM}$ potential eq. 63 from fermions $\left\{\mathbf{r}_{Q}, \mathbf{r}_{T}\right\}=\left\{\mathbf{2 0}^{\prime}, \mathbf{1}\right\}$. The individual contributions of the $\mathrm{SO}(6) / \mathrm{SO}(4) \times \mathrm{SO}(2)$ operators of table 3 are shown. The first line indicates the NDA pre-factor.

\section{Almost Inert Higgs}

Even if some terms in the Lagrangian, such as the up Yukawas previously discussed, are accidentally $C_{2}$-invariant, one would naively expect maximal breaking of $C_{2}$ elsewhere, in particular in the potential, given that the strong sector by assumption breaks $C_{2}$. That would force both $\Phi_{\widehat{1}}$ and $\Phi_{\widehat{2}}$ to acquire a VEV, and this compatibly with unbroken $C_{1} P$. At leading order $\left(\propto y_{L}^{2}\right)$, however, the only three $C_{1} P$ even contributions to the potential are also $C_{2}$-even, as table 3 shows. The potential is thus accidentally approximately $C_{2}$ invariant. Furthermore, we have found that the leading $C_{2}$-odd contribution (i.e., $C_{1} P$ even) comes at order $y_{L}^{4}$. The $C_{2}$-odd mass $m_{12}^{2}$ that controls the $\left\langle\Phi_{\widehat{\mathbf{z}}}\right\rangle$ is therefore

$$
m_{12}^{2} \sim \frac{N_{c}}{16 \pi^{2}} y_{L}^{4} f^{2} \simeq(70 \mathrm{GeV})^{2}\left(\frac{y_{L}}{1}\right)^{4}\left(\frac{0.25}{\xi}\right) .
$$

The associated phenomenological implications will be discussed in the section 4.4.2.

The contributions to the renormalizable potential in eq. 63 arising from each of the three allowed operators are shown in table 7, from which several interesting consequences can be drawn. First, we see that the leading order potential is tunable, without need of including sub-leading corrections. This is clearly an advantage as compared with the $\{\mathbf{6 , 6}\}$ model of the previous section, and also the MCHM [2]: in the model at hand less fine-tuning is required to reach the same value of $\xi$. Second, we see that there is a unique contribution to both $m_{22}^{2}$ and $\lambda_{2}$; their ratio being fixed, makes not possible to tune the $\operatorname{VEV}$ of $\Phi^{\widehat{2}}$. This means that the VEV of $\Phi^{\widehat{2}}$ cannot be fine-tuned to be smaller than $f$, so that even if 
a $C_{2}$-breaking vacuum existed, it would be difficult to make it phenomenologically viable. Third, one can check, by studying explicitly the leading order potential, that a stable vacuum with both $\Phi^{\widehat{1}}$ and $\Phi^{\widehat{2}}$ taking a VEV does not exist. Therefore not only we know that a $C_{2}$-preserving vacuum exists (because of the accidental $C_{2}$ symmetry), but we also see that spontaneous $C_{2}$ breaking cannot be achieved by the leading order potential. The model is therefore "forced" to resemble the Inert Higgs.

In this setup, differently from the one of the previous section, the quartic $\lambda_{1}$ is not reduced by the tuning and the Higgs mass therefore reads

$$
m_{h}^{2} \sim \frac{N_{c}}{16 \pi^{2}} y_{L}^{2} g_{\rho}^{2} v^{2} \simeq(250 \mathrm{GeV})^{2}\left(\frac{y_{L}}{1}\right)^{2}\left(\frac{3}{N}\right) .
$$

The masses of the other scalars, the $\mathrm{SO}(3)_{c}$ triplet, $H^{a}$, and singlet, $H$, are dominated by a common $\mathrm{SO}(4)$-symmetric contribution

$$
m_{H_{2}}^{2} \sim \frac{N_{c}}{16 \pi^{2}} y_{L}^{2} g_{\rho}^{2} f^{2} \simeq(500 \mathrm{GeV})^{2}\left(\frac{y_{L}}{1}\right)^{2}\left(\frac{3}{N}\right)\left(\frac{0.25}{\xi}\right) .
$$

After EWSB, $H$ gets an additional contribution through the $\lambda_{4}$ coefficient. We can calculate this tripletsinglet mass splitting using table 7. At order $y^{2}$ we have only three operators and therefore the potential depends only on their three unknown coefficients. The tuning $v \ll f$ gives an approximate relation between the three coefficients, which can be used to eliminate one. The other two can be traded for $m_{h}$ and $m_{H^{a}}$. We can then obtain a prediction for $m_{H}$ as a function of these masses:

$$
\frac{m_{H}^{2}-m_{H^{a}}^{2}}{m_{H}^{2}} \simeq \frac{1}{3}\left(\frac{m_{h}^{2}}{m_{H}^{2}}+\xi\right) \sim \xi .
$$

Custodial-breaking splitting comes from gauge contributions $\left(\propto g^{\prime 2}\right)$, and higher orders in $y_{L}\left(\propto y_{L}^{4}\right)$. These splittings can be estimated as

$$
\begin{aligned}
& \left|\frac{m_{H^{ \pm}}-m_{A}}{m_{H^{ \pm}}}\right|_{g^{\prime}} \sim\left(\frac{g^{\prime}}{y_{L}}\right)^{2} \xi \simeq 0.03\left(\frac{1}{y_{L}}\right)^{2}\left(\frac{\xi}{0.25}\right), \\
& \left|\frac{m_{H^{ \pm}}-m_{A}}{m_{H^{ \pm}}}\right|_{y_{L}^{4}} \sim\left(\frac{y_{L}}{g_{\rho}}\right)^{2} \xi \simeq 0.005\left(\frac{y_{L}}{1}\right)^{2}\left(\frac{N}{3}\right)\left(\frac{\xi}{0.25}\right) .
\end{aligned}
$$

\subsection{Extended Custodial Symmetry}

In this section we wish to briefly discuss a last possibility, where large corrections to $\widehat{T}$ are avoided thanks to an $\mathrm{SU}(2)^{3}$ custodial symmetry in the Higgs sector. That symmetry allows arbitrary Higgs VEVs to preserve a diagonal $\mathrm{SU}(2)=\mathrm{SO}(3)_{c}$ which guarantees that $\widehat{T}=0$ at leading order. The simplest realization of the idea is provided by the coset

$$
\frac{\mathrm{Sp}(6)}{\mathrm{SU}(2) \times \mathrm{Sp}(4)},
$$

which delivers $8 \mathrm{NGB}$ in the $(\mathbf{2}, \mathbf{4})$ representation of the unbroken group, corresponding to two Higgs doublets. The unbroken symmetry coincides with the one of the renormalizable 2HDM after gauging 
$\mathrm{SU}(2)_{L}$. This is easily seen by embedding the two Higgses $\Phi^{\widehat{1}, \widehat{2}}$, in the $2 \times 2$ matrix notation, into a $2 \times 4$ matrix

$$
M=\left(\Phi^{\widehat{1}}, \Phi^{\widehat{2}}\right)
$$

If $M$ was a generic matrix, we could act on it with an $\mathrm{SU}(2)$ rotation on the left (which correspond to the $\mathrm{SU}(2)_{L} \mathrm{SM}$ group) and with an element of $\mathrm{SU}(4)$ on the right. The renormalizable 2HDM Lagrangian, once rewritten in terms of the matrix $M$, is immediately seen to be invariant under this $\mathrm{SU}(2) \times \mathrm{SU}(4)$ group. The pseudo-reality condition of $\Phi^{\widehat{1}, \widehat{2}}$, however, implies

$$
M^{*}=\sigma_{2} M \Sigma_{2}
$$

where $\Sigma_{2}=\operatorname{diag}\left(\sigma_{2}, \sigma_{2}\right)$. The above condition breaks the group of allowed transformations to $\mathrm{SU}(2) \times$ $\operatorname{Sp}(4)$, as was to be shown.

This mechanism could be also be extended to $N$ Higgses. Here the relevant coset is

$$
\frac{\mathrm{Sp}(2 N+2)}{\mathrm{SU}(2) \times \operatorname{Sp}(2 N)} \text {. }
$$

which produces $N$ doublets. The group $H$ is the symmetry group of the renormalizable model, and contains the subgroup $\mathrm{SU}(2)^{N+1}$ which protects the $\rho$ parameter in the case of $N$ Higgs doublets.

We will focus on the $N=2$ coset in what follows. Under the extended custodial subgroup $\mathrm{SU}(2)_{L} \times$ $\mathrm{SU}(2)_{R 1} \times \mathrm{SU}(2)_{R 2}$ of $H=\mathrm{SU}(2) \times \mathrm{Sp}(4)$ the NGB decompose as follows,

$$
\begin{aligned}
& \Phi^{\widehat{1}}=(2,2,1), \\
& \Phi^{\widehat{2}}=(2,1,2) .
\end{aligned}
$$

We identify the hypercharge with the linear combination

$$
Y=T_{R 1}^{3}+T_{R 2}^{3}+X
$$

where the extra $\mathrm{U}(1)_{X}$ charge $X$ is needed to obtain the correct fermionic hypercharges, while the Higgs is $X$-neutral. By the above choice, the two Higgs doublets have the same hypercharge.

Let us now turn to the fermions. The smallest representations of $\operatorname{Sp}(6)$ decompose under $H$ as

$$
\begin{gathered}
\mathbf{6}=(\mathbf{2}, \mathbf{1}) \oplus(\mathbf{1}, \mathbf{4}), \\
\mathbf{1 4}=(\mathbf{1}, \mathbf{1}) \oplus(\mathbf{1}, \mathbf{5}) \oplus(\mathbf{2}, \mathbf{4}), \\
\mathbf{1 4}^{\prime}=(\mathbf{1}, \mathbf{4}) \oplus(\mathbf{2}, \mathbf{5}), \\
\mathbf{2 1}=(\mathbf{3}, \mathbf{1}) \oplus(\mathbf{1}, \mathbf{1 0}) \oplus(\mathbf{2}, \mathbf{4}) .
\end{gathered}
$$

We see that several possibilities exist for embedding the SM doublets and singlets. The safer option for EWPT is, as discussed in section 2.4, to embed the $t_{R}$ in a singlet of the custodial group, so that the $y_{R}$ proto-Yukawa does not contribute to $\widehat{T}$, and to take the limit of total $t_{R}$ compositeness $y_{R} \rightarrow g_{\rho}$. This 
is because in that case we can take a small $y_{L} \simeq Y_{t}$ thus controlling $\widehat{T}$ and $\delta g_{b} / g_{b}$. Those considerations easily generalize to the present case, though we should remember that the custodial group is not $\mathrm{SO}(4)$, but the larger $\mathrm{SU}(2)_{L} \times \mathrm{SU}(2)_{R 1} \times \mathrm{SU}(2)_{R 2}$ group. In particular $t_{R}$ should be a complete singlet of the latter group. On the other hand, it is not immediately evident to see whether $P_{L R}$ plays a role in further suppressing $\delta g_{b} / g_{b}$, as discussed in section 2.4. In the affirmative, one could depart from the limit of total $t_{R}$ compositeness, but we will ignore this possibility in the following.

Compatibly with the previous discussion, $t_{R}$ could either be embedded in a 14, or $\mathbf{2 1}$ or in a total singlet, with $X=2 / 3$ charge. Let us choose for definiteness the case of the $\mathbf{1}_{\mathbf{2} / \mathbf{3}}$, while for the $q_{L}$ doublet we pick a $\mathbf{1 4}_{\mathbf{2} / \mathbf{3}}$. Within the $\mathbf{1 4}_{\mathbf{2} / \mathbf{3}}, q_{L}$ can be embedded in either the $(\mathbf{2}, \mathbf{2}, \mathbf{1})_{\mathbf{2} / \mathbf{3}}$ or the $(\mathbf{2}, \mathbf{1}, \mathbf{2})_{\mathbf{2} / \mathbf{3}}$ of $\mathrm{SU}(2)_{L} \times \mathrm{SU}(2)_{R 1} \times \mathrm{SU}(2)_{R 2}$ (which are in turn contained in the the $(\mathbf{2}, \mathbf{4})_{\mathbf{2} / \mathbf{3}}$ of $\mathrm{SU}(2)_{L} \times \mathrm{Sp}(4)$ ). Concerning flavor, applying the general consideration of section 2.3 , this model behaves similarly to the one with the $\mathbf{2 0}^{\prime}$ and the singlet discussed in section 3.1.3. There is a unique Yukawa structure, because a unique $H$ singlet can be formed among the $\mathbf{1 4}$ and the singlet (see eq. 89), but two embeddings are present for the $q_{L}$ in the $\mathbf{1 4}$, as previously discussed. As in the model of section 3.1.3, in order to avoid Higgs-mediated FCNC, the embeddings must be taken flavor independent.

The above discussion shows that no obstructions seems to arise when trying to construct explicit models with an extended custodial group. A detailed phenomenological study of the model we have just described, and of the other possibilities that may be envisaged in the context of the $\mathrm{Sp}(6) / \mathrm{SU}(2) \times \mathrm{Sp}(4)$ coset, lies however outside the scope of the present paper.

\section{Phenomenology of Composite 2HDM}

In this section we will study the phenomenological implications of the composite 2HDM, starting with the generic properties, and focussing later on the explicit examples we constructed. For this purpose we will derive the effective Lagrangian describing Higgs physics at energies below $m_{\rho}$. That can be written in terms of an expansion in powers of $H_{i} / f$ and $\partial_{\mu}^{2} / m_{\rho}^{2}$. The leading dimension- 4 operators describe the Lagrangian of an elementary (i.e. renormalizable) 2HDM. It is essential, for the purpose of the following discussion, to recall the properties of that limiting case.

\subsection{Elementary 2HDM}

The Lagrangian of the elementary 2HDM, focussing just on the top Yukawa terms, can be written as

$$
\mathcal{L}=\left|D_{\mu} H_{1}\right|^{2}+\left|D_{\mu} H_{2}\right|^{2}+Y_{t} \bar{q}_{L}\left(\tilde{H}_{1}+a_{t} \tilde{H}_{2}\right) t_{R}+\text { h.c. }+V\left(H_{1}, H_{2}\right) .
$$

It will be convenient in this section to work in the basis in which only one doublet acquires a VEV. In the unitary gauge this is given by

$$
H_{1}=\left(\begin{array}{c}
0 \\
\left(v+h^{0}\right) / \sqrt{2}
\end{array}\right), \quad H_{2}=\left(\begin{array}{c}
H^{+} \\
\left(H^{0}+i A^{0}\right) / \sqrt{2}
\end{array}\right) .
$$


In this basis the mass eigenstates, in the limit where $C P$ is not violated, are

$$
h_{1}^{0}=h^{0} \cos \theta_{h}+H^{0} \sin \theta_{h}, \quad h_{2}^{0}=-h^{0} \sin \theta_{h}+H^{0} \cos \theta_{h}, \quad A^{0} \quad \text { and } \quad H^{ \pm},
$$

where $\theta_{h}$ is determined by the potential $V\left(H_{1}, H_{2}\right)$. The angle $\theta_{h}$ is obviously proportional to the source of $C_{2}$ breaking, but also to $v^{2}$. That is because in the limit of unbroken $\mathrm{SU}(2)_{L}$, i.e. $v \rightarrow 0, H^{0}$ should not mix with $h^{0}$ and become degenerate with $A^{0}$ and $H^{+}$. Therefore in composite 2HDM one expects $\theta_{h} \propto(v / f)^{2}$, possibly times an extra reduction factor associated to the small breaking of $C_{2}$.

The specific form of the kinetic terms in eq. (90), when expressed in terms of the mass eigenstate fields, leads to a set of relations between the masses of vector bosons and their coupling to scalars. Assuming $C P$ invariance, those can be written as the sum rules

$$
\begin{aligned}
S_{\phi V V} & \equiv \frac{\sum_{i} g_{h_{i}^{0} W^{+} W^{-}}^{2}}{g^{2} m_{W}^{2}}=\frac{\sum_{i} g_{h_{i}^{0} Z Z}^{2}}{g^{2} m_{Z}^{2} / c_{W}^{2}}=\frac{\sum_{i} g_{h_{i}^{0} W^{+} W^{-}} g_{h_{i}^{0} Z Z}}{g^{2} m_{Z}^{2}}=1 \\
S_{\phi \phi V} & \equiv \frac{\sum_{i} g_{h_{i}^{0} H^{+} W^{-}}^{2}}{g^{2}}=\frac{\sum_{i} g_{h_{i}^{0} A^{0} Z}^{2}}{g^{2} c_{W}^{2}}=\frac{\sum_{i} g_{h_{i}^{0} H^{+} W^{-}} g_{h_{i}^{0} A^{0} Z}}{g^{2} / c_{W}}=1 \\
S_{\phi V} & \equiv \frac{\sum_{i} g_{h_{i}^{0} W^{+} W^{-}} g_{h_{i}^{0} A^{0} Z}}{g^{2} m_{W} / c_{W}}=\frac{\sum_{i} g_{h_{i}^{0} W^{+} W^{-}} g_{h_{i}^{0} H^{+} W^{-}}}{g^{2} m_{W}}=\frac{\sum_{i} g_{h_{i}^{0} Z Z} g_{h_{i}^{0} A^{0} Z}}{g^{2} m_{Z} / c_{W}^{2}}=\frac{\sum_{i} g_{h_{i}^{0} Z Z} g_{h_{i}^{0} H^{+} W^{-}}}{g^{2} m_{Z} / c_{W}}=0 .
\end{aligned}
$$

and as the relations

$$
\begin{array}{r}
\frac{g_{A^{0} H^{+} W^{-}}^{2}}{g^{2} / 4}=\frac{g_{H^{+} H^{-} Z}^{2}}{g^{2} / 4 c_{W}^{2}}=1, \\
g_{H^{+} W^{-} Z}=0,
\end{array}
$$

where the sums run over the two neutral $C P$-even scalars $h_{1}^{0}$ and $h_{2}^{0}$, while, with an obvious notation, the interaction vertices are defined by

$$
\begin{aligned}
\mathcal{L}_{\text {int }}^{\phi V_{a} V_{b}} & =g_{\phi V^{a} V^{b}} \phi V_{\mu}^{a} V_{b}^{\mu}, \\
\mathcal{L}_{\text {int }}^{\phi \phi^{\prime} V_{a}} & =g_{\phi \phi^{\prime} V^{a}} \phi \overleftrightarrow{\partial}_{\mu} \phi^{\prime} V_{a}^{\mu}
\end{aligned}
$$

It is noteworthy the vanishing of $g_{H^{+} W^{-} Z}$ at the renormalizable level. A further set of useful relations following from the sum rules is

$$
\frac{g_{h_{1}^{0} Z Z}}{g_{h_{2}^{0} A^{0} Z}}=-\frac{g_{h_{2}^{0} Z Z}}{g_{h_{1}^{0} A^{0} Z}}=m_{Z}
$$

and similarly for ratios involving $g_{h_{i}^{0} W^{+} W^{-}}$and $g_{h_{j}^{0} H^{+} W^{-}}$, with $i \neq j$. 
In the very same way the scalar couplings to the top multiplet satisfy specific relations. In the mass eigenstate basis the Yukawa interactions read (in the $C P$ conserving limit)

$$
\mathcal{L}_{\text {top }}=Y_{t}\left\{\frac{1}{\sqrt{2}} \bar{t} t\left[\left(\cos \theta_{h}+a_{t} \sin \theta_{h}\right) h_{1}^{0}+\left(a_{t} \cos \theta_{h}-\sin \theta_{h}\right) h_{2}^{0}\right]+\frac{i a_{t}}{\sqrt{2}} \bar{t} \gamma_{5} t A^{0}+a_{t}\left(\bar{b}_{L} t_{R} H^{-}+\text {h.c. }\right)\right\}
$$

where only one parameter, $a_{t}$, accounts for four couplings. This results in three relations that can be written as

$$
\begin{aligned}
y_{h_{1}^{0}} \cos \theta_{h}-y_{h_{2}^{0}} \sin \theta_{h} & =\frac{m_{t}}{v}, \\
y_{h_{1}^{0}}^{2}+y_{h_{2}^{0}}^{2}-\left(\frac{m_{t}}{v}\right)^{2} & =y_{A^{0}}^{2}=\frac{y_{H^{+}}^{2}}{2} .
\end{aligned}
$$

The above relations follow from a renormalizable, weakly coupled Lagrangian. Their violation leads to a growth with energy for the scattering amplitudes involving scalars and/or longitudinally polarized vectors. In the case of our PNGB, the growth of the scattering amplitudes is dictated by the $\sigma$-model derivative interactions.

\subsection{Composite 2HDM}

We want now to discuss how things change in the composite $2 \mathrm{HDM}$ when including higher-dimensional operators. Among these operators, those involving the Higgs fields without any derivative, for example $\left|H_{1}\right|^{2}\left|H_{2}\right|^{4} / f^{2}$, will not be relevant for us since they only modify the potential $V\left(H_{1}, H_{2}\right)$, but obviously not the relations derived in the previous section. Higher derivative terms are suppressed by $O\left(\partial_{\mu}^{2} / m_{\rho}^{2}\right)$ and thus normally subleading, at sufficiently low energies, with respect to the $O\left(v^{2} / f^{2}\right)$ effects coming from non-linear 2-derivatives scalar interactions [5]. Let us first comment on the general case, i.e. working to all order in $v / f$ and without assuming a specific $\sigma$-model structure.

In the general case, unlike in the renormalizable one, we cannot find an operator basis that diagonalizes the kinetic terms, upon expansion, at any point in the field space. Because of that, in any parametrization where $\left\langle H_{2}\right\rangle=0$ there will still be kinetic mixings between the components of $H_{2}$ and the NGB living inside $H_{1}$. In this situation the standard unitary gauge of eq. (91) is no longer eliminating the bilinear mixings between vectors and scalars. Assuming custodial invariance the "canonical" parametrization is of the form

$$
H_{1}=\left(\begin{array}{c}
\epsilon_{T} H^{+} \\
\left(v+h^{0}+\epsilon_{H} H^{0}+i \epsilon_{T} A^{0}\right) / \sqrt{2}
\end{array}\right), \quad H_{2}=\left(\begin{array}{c}
H^{+} \\
\left(H^{0}+i A^{0}\right) / \sqrt{2}
\end{array}\right),
$$

where $\epsilon_{T}$ is fixed by the gauge choice so as to eliminate the mixing of $H^{+}$and $A^{0}$ with vectors, while $\epsilon_{H}$ is chosen by requiring vanishing kinetic $h^{0}-H^{0}$ mixing (though these, in general, are not yet mass eigenstates). The above represents the general case. However in the specific case where the kinetic terms respect an $\mathrm{SO}(2)$ symmetry under which $\left(H_{1}, H_{2}\right)$ form a doublet, it is easy to see that $\epsilon_{T}=\epsilon_{H}=0$. Moreover, assuming $\mathrm{SO}(4)$ invariance, but not $\mathrm{SO}(2)$, and limiting the analysis to dimension 6 operators, one is not forced to use the general parametrization of eq. (101). Indeed, as shown in Appendix A, 
by performing the most general field redefinition of the form $H \rightarrow H+H^{3} / f^{2}$ one can reduce the set of dimension 6 terms to the eight operators listed in eq. (127). In principle after the non-linear field redefinition both $H_{1}$ and $H_{2}$ will have a non vanishing VEV, but it is easy to see that the basis (not the individual operators) in eq. (127) is invariant under simple rotations in the $\left(H_{1}, H_{2}\right)$ plane. By one such rotation we can therefore always choose $\left\langle H_{2}\right\rangle=0$. By inspecting the operators in eq. 127) one finds $\epsilon_{T}=0$ and $\epsilon_{H}=-\left(c_{H_{1} H_{12}} / 2\right)\left(v^{2} / f^{2}\right)$.

Substituting eq. 101 in the operators of eq. 127) we obtain corrections of order $v^{2} / f^{2}$ to the Higgs couplings. The relevant ones for the trilinear couplings are

$$
\begin{aligned}
\mathcal{L}_{3-i n t}= & \frac{g m_{W}}{2}\left[\left(1+\frac{v^{2}}{f^{2}} c_{1}\right) h^{0}+\frac{v^{2}}{f^{2}} c_{2} H^{0}\right] V_{a}^{\mu} V_{\mu}^{a}+i g\left(1+\frac{v^{2}}{f^{2}} c_{3}\right) H^{0} V_{a}^{\mu} \partial_{\mu} H^{a} \\
& +\frac{g}{2}\left(1+\frac{v^{2}}{f^{2}} c_{4}\right) \epsilon_{a b c} H^{a} \partial^{\mu} H^{b} A_{\mu}^{c}+\frac{v}{f^{2}} c_{5} h^{02} \partial_{\mu}^{2} H^{0}
\end{aligned}
$$

where

$$
\begin{aligned}
V_{a}^{\mu} V_{\mu}^{a} & \equiv 2\left(W^{+}\right)_{\mu}\left(W^{-}\right)^{\mu}+\frac{1}{\cos ^{2} \theta_{W}} Z_{\mu} Z^{\mu} \\
i V_{a}^{\mu} \partial_{\mu} H^{a} & \equiv i W^{-\mu} \partial_{\mu} H^{+}+\text {h.c. }+\frac{1}{\cos \theta_{W}} Z^{\mu} \partial_{\mu} A^{0} \\
\epsilon_{a b c} H^{a} \partial^{\mu} H^{b} V_{\mu}^{c} & \equiv \frac{i}{\cos \theta_{W}} H^{-} \partial^{\mu} H^{+} Z_{\mu}+A^{0} \partial^{\mu} H^{-} W_{\mu}^{+}-H^{+} \partial^{\mu} A^{0} W_{\mu}^{-}+\text {h.c. }
\end{aligned}
$$

and

$$
c_{1}=-c_{H_{1}}, c_{2}=-\frac{1}{2} c_{H_{1} H_{12}}, c_{3}=-\frac{1}{4} c_{H_{12}}+c_{T}, c_{4}=c_{\epsilon}, c_{5}=-\frac{1}{2} c_{H_{1} H_{12}}
$$

in terms of the coefficients of the dimension 6 operators in eq. 127). In eq. 102, terms proportional to $\partial_{\mu} V^{\mu}$ have been omitted since they do not play any role in the production or decay of the Higgs bosons. Notice also the absence of $H^{+} W^{-} Z$ and $A^{0} V^{a} V^{a}$ couplings that, as will be discussed in more details below, is a consequence of custodial symmetry. We recall that $h^{0}$ and $H^{0}$ are not in general physical states, since they mix at order $\theta_{h} \sim v^{2} / f^{2}$ in the potential.

The corrections to the vertices of eq. 102 modify the relations 93 and 94 . We now obtain

$$
\begin{aligned}
S_{\phi V V} & =\left(1+2 c_{1} \frac{v^{2}}{f^{2}}\right), \\
S_{\phi \phi V} & =\left(1+2 c_{3} \frac{v^{2}}{f^{2}}\right), \\
S_{\phi V} & =c_{2} \frac{v^{2}}{f^{2}}, \\
\frac{g_{A^{0} H^{+} W^{-}}^{2}}{g^{2} / 4} & =\frac{g_{H^{+} H^{-} Z}^{2}}{g^{2} / 4 c_{W}^{2}}=\left(1+2 c_{4} \frac{v^{2}}{f^{2}}\right) .
\end{aligned}
$$


The ratios eq. (97) get also modified:

$$
\frac{g_{h_{1}^{0} Z Z}}{g_{h_{2}^{0} A Z}}=m_{Z}\left[1+\left(c_{1}-c_{3}\right) \frac{v^{2}}{f^{2}}\right], \quad \frac{g_{h_{2}^{0} Z Z}}{g_{h_{1}^{0} A Z}}=-m_{Z}\left[1-c_{2} \frac{1}{\tan \theta_{h}} \frac{v^{2}}{f^{2}}\right] .
$$

Similarly to the genuine $\sigma$-model corrections to the purely bosonic interactions, we can study the implications of compositeness on the interactions between scalars and fermions. As already emphasized in ref. [5], for approximately elementary fermions the leading effects come from "higher order" Yukawa interactions, obtained by sprinkling powers of $H_{1}$ on the leading order result. Sticking to the parametrization $\left\langle H_{2}\right\rangle=0$ and focussing on terms that affect trilinears, we have three new dimension 6 operators

$$
Y_{t}\left(\bar{q}_{L} \tilde{H}_{1} t_{R}\right)\left[\frac{c_{t}^{(1)}}{f^{2}} H_{1}^{\dagger} H_{1}+\frac{c_{t}^{(2)}}{f^{2}} H_{1}^{\dagger} H_{2}+\frac{c_{t}^{(3)}}{f^{2}} H_{2}^{\dagger} H_{1}\right]+Y_{t} a_{t} \frac{c_{t}^{(4)}}{f^{2}}\left(\bar{q}_{L} \tilde{H}_{2} t_{R}\right) H_{1}^{\dagger} H_{1}+\text { h.c. },
$$

where $c_{t}^{(i)}$ are $O(1)$ coefficients. Because of these four new coefficients all three relations in eq. 100 are modified at order $v^{2} / f^{2}$.

Another potentially interesting implication of higher-derivative terms involving PNGB and fermions arises in the class of models where accidental symmetries appear at lowest order in the action, as those discussed in section 3 . For example, in the particular model of section 3.1 .3 where $C_{2}$ is badly broken in the strong sector, but accidentally preserved in the zero derivative terms of the scalar potential and Yukawa interactions, it is interesting to investigate whether this breakdown shows up unsuppressed in the derivative interactions between PNGB and composite fermions. To be definite let us focus on the case in which $t_{R}$ is a fully composite object. In principle we could expect terms involving the current $\bar{t}_{R} \gamma^{\mu} t_{R}$ and NGB currents. In the case $\mathrm{SO}(6) / \mathrm{SO}(4) \times \mathrm{SO}(2)$ one can quickly see that no such term can be written. Since the $\mathcal{D}_{\mu}^{i \alpha}$ is a $(\mathbf{4}, \mathbf{2})$ of $\mathrm{SO}(4) \times \mathrm{SO}(2)$, an invariant term must involve a top current with the same quantum numbers, which is not the case in any model we constructed, and probably of any sensible model in general. The simplest seemingly sensible possibility is to have $t_{R}$ charged under $\mathrm{SO}(2)$ in which case the covariant derivative

$$
\bar{t}_{R} \gamma^{\mu}\left(\partial_{\mu}+\mathcal{E}_{\mu}\right) t_{R}
$$

would break $C_{2}$ and give rise to a term $\bar{t}_{R} \gamma^{\mu} t_{R} \Phi_{\widehat{1}} \overleftrightarrow{\partial}_{\mu} \Phi_{\widehat{2}}$ upon expanding in the NGB fields. Unfortunately, in the model with $\left\{\mathbf{2 0}^{\prime}, \mathbf{1}\right\}$ we have that $t_{R}$ can be fully composite but is a singlet, while in the model with $\{\mathbf{6}, \mathbf{6}\}$ we have that $t_{R}$ is not a singlet but the symmetry $C_{2}$ is preserved by the strong interactions and therefore the term in eq. (108) cannot be generated. As a last remark we notice that in $\mathrm{SO}(6) / \mathrm{SO}(4)$ there is an additional NGB transforming as a singlet of $\mathrm{SO}(4)$. The corresponding $\mathcal{D}_{\mu}$ can be coupled to the right-handed top current and generates a sizable effect. But again this theory has an additional neutral scalar and it is not just a 2HDM.

\section{3 $\quad H^{ \pm} W^{\mp} Z$ and the Role of Symmetries}

One might have hoped that the richer kinetic structure of these models would allow for the presence of interactions which, in a renormalizable theory, are absent at tree-level. This is for example the case of 
$H^{ \pm} W^{\mp} Z$, which would be a golden channel for $H^{+}$detection. In the renormalizable $2 \mathrm{HDM}$, the $H^{ \pm} W^{\mp} Z$ vertex is generated only at the one-loop level [15]. Unfortunately, the situation is not much better in realistic composite models, due to the custodial $\mathrm{SO}(3)$ that plays a crucial role. To analyze this, we will use the CCWZ construction of the $\mathrm{SO}(4) / \mathrm{SO}(3) \sigma$-model effective Lagrangian obtained after EWSB, in full analogy with the discussion of $Z b \bar{b}$ in section 2.4. Let us start with operators involving only the custodial triplets $\mathcal{D}_{\mu}^{a}, \mathcal{E}_{\mu}^{a}$ and $H^{a}$. At the two derivative level the only object one can write is

$$
\left(\partial^{\mu}+\mathcal{E}^{\mu}\right) H^{a} \mathcal{D}_{\mu}^{a}
$$

where $\mathcal{E}_{\mu}$ contains both NGB and gauge fields, upon weak gauging of the global symmetry. The above term however induces a kinetic mixing between the electroweak gauge bosons and the heavy Higgs triplet $H^{a}$. As a consequence this term will be eliminated by the suitable (physical) gauge choice for which no such mixing exists. Another possible two derivative operator $\mathcal{D}_{\mu}^{a} \mathcal{D}^{b \mu} H^{c} \epsilon_{a b c}$ vanishes by Bose symmetry. It is also similarly easy to deal with operators that involve $H^{a}$ and two powers of $\mathcal{D}_{\mu}^{a}$, plus a number of covariant derivatives acting on them. These would correspond to effects that are genuinely associated to the strong sector, and thus only involve the longitudinally polarized vectors. Zooming on the trilinear interactions we can replace $\mathcal{D}_{\mu}^{a}=\partial_{\mu} G^{a}$, where $G^{a}$ is the triplet of SM NGB fields. Then, integrating by parts, and using the lowest order equation of motion $\square G^{a}=0$ such operators can always be written as

$$
H^{a}\left(\partial_{\mu} \ldots \partial_{\nu} G^{b}\right)\left(\partial^{\mu} \ldots \partial^{\nu} G^{c}\right)
$$

Again the contraction with $\epsilon_{a b c}$, which would lead to a singlet, vanishes. This means that the only contribution to $H^{ \pm} W^{\mp} Z$ can only come from the terms we neglected in the above procedure: custodial breaking terms (from the NGB equations of motion) and terms explicitly involving gauge fields. The latter survive only to the extent that the gauge field configuration is not gauge equivalent to a NGB field, so they must necessarily involve the gauge field strength and therefore will lead to effects suppressed by powers of $\partial_{\mu}^{2} / m_{\rho}^{2}$. We conclude then that the contributions to $H^{ \pm} W^{\mp} Z$ should involve either custodial breaking spurions or gauge field strengths or both. Before considering these other effects, we must point out that for symmetric cosets, like $\mathrm{SO}(6) / \mathrm{SO}(4) \times \mathrm{SO}(2)$, there are no interaction terms involving an odd number of NGB. In those cases it is trivial to realize that $H^{ \pm} W^{\mp} Z$ is not enhanced by pure $\sigma$-model terms involving the eaten NGBs. The proof we gave in this paragraph is however more general, as it solely relys on $\mathrm{SO}(3)$ invariance.

Let us now consider terms with field strengths. The lowest-order terms involve just one field strength, and we find two such terms

$$
\mathcal{O}_{B}=\frac{1}{m_{\rho}^{2}} D_{\mu} H^{a} \mathcal{D}_{\nu}^{a} B^{\mu \nu} \quad, \quad \mathcal{O}_{W}=\frac{1}{m_{\rho}^{2}} D_{\mu} H^{a} \mathcal{D}_{\nu}^{b} W^{c \mu \nu} \epsilon_{a b c}
$$

One further question concerns the order at which these terms arise in our $\mathrm{SO}(6) / \mathrm{SO}(4) \times \mathrm{SO}(2)$ cosets. To investigate that, we must work with the $\mathrm{SO}(6) / \mathrm{SO}(4) \times \mathrm{SO}(2)$ NGB fields $\mathcal{D}_{\mu}^{\alpha i}$. In doing so one is easily 
convinced that, because of Bose symmetry, only the first operator survives

$$
\frac{1}{m_{\rho}^{2}} \epsilon^{\alpha \beta} \mathcal{D}_{\mu}^{\alpha i} \mathcal{D}_{\nu}^{\beta i} B^{\mu \nu}
$$

Of course this operator is only generated if the strong sector breaks $C_{2}$. Notice also that $B_{\mu \nu}$, from the point of view of the strong sector, plays the role of the field strength of the weakly gauged $\mathrm{U}(1)_{X}$. In that sense the presence of $C_{X}$ charge conjugation within the strong sector would forbid that term. The above operator eq. 112 contributes to both $H^{+} \rightarrow W^{+} Z$ and to the more interesting $H^{+} \rightarrow W^{+} \gamma$. Unfortunately, assuming minimal coupling [5] (as it is the case in five-dimensional realization of these models), the coefficient of the operators $(111)$ is further suppressed by $g_{\rho}^{2} / 16 \pi^{2}$, and the phenomenological relevance of these decay modes is very limited.

Let us now consider effects induced by the custodial-breaking spurions. We have two of them, the top proto-Yukawa $y_{L}$ and the gauge coupling $g^{\prime}$. As already discussed in section 3 , only combinations that are invariant under the additional rephasing of the external fields can enter in the strong-sector Lagrangian. These are respectively $\Upsilon_{L}$ of eq. 49 that contains a $\mathrm{SO}(3)$ triplet, $w^{a}$, with VEV along $a=3$, and $\Gamma_{g^{\prime}}^{ \pm}$ of eq. (55) that contains a $\mathrm{SO}(3)$ triplet and a quintuplet, $w^{a b}$ (a traceless symmetric tensor), with VEV along the 33 component. In classifying the operators $C P$ plays a crucial role. For this purpose it is useful to recall, as discussed in section 3 , that on the bosonic fields $C P$ reduces to just parity times $C_{1}$, a 180 degree rotation in the 1-3 plane of $\mathrm{O}(4)$ defined in eq. (13). In view of that, $w^{3}$ and $w^{33}$ are respectively $C_{1}$ odd and $C_{1}$ even. Operators with odd powers of $w^{a}$ will break $C P{ }^{16}$. Now at the two derivative level we find the following terms

$$
\begin{aligned}
\mathcal{O}_{3} & =\frac{v}{f^{2}} \frac{w^{a}}{16 \pi^{2}} \mathcal{D}_{\mu}^{a} H^{b} \mathcal{D}^{b \mu}, \quad \mathcal{O}_{4}=\frac{v}{f^{2}} \frac{w^{a}}{16 \pi^{2}} H^{a} \mathcal{D}_{\mu}^{b} \mathcal{D}^{b \mu} \\
\mathcal{O}_{5} & =\frac{v}{f^{2}} \frac{w^{a b}}{16 \pi^{2}} H^{c} \mathcal{D}_{\mu}^{a} \mathcal{D}^{d \mu} \epsilon^{b c d}
\end{aligned}
$$

as well as a term of the same form as $\mathcal{O}_{5}$ but with $w^{a b}$ replaced by $w^{a} w^{b} / g_{\rho}^{2}$. For $y_{L} \sim Y_{t}$ that term is however subleading. $\mathcal{O}_{3,4}$ can only be generated if $C P$ is broken by the strong sector. They lead to the $C P$-odd coupling

$$
i Z_{\mu}\left(H^{+} W^{-\mu}-H^{-} W^{+\mu}\right)
$$

together with different combinations of $A^{0} W_{\mu}^{+} W^{-\mu}$ and $A^{0} Z_{\mu} Z^{\mu}$. We recall that our conventions on $C P$ are $H^{ \pm}, W_{\mu}^{ \pm} \rightarrow H^{\mp}, W_{\mu}^{\mp}$ and $Z_{\mu} \rightarrow-Z_{\mu}$. On the other hand $\mathcal{O}_{5}$ respects $C P$ and will be generically present leading just to the trilinear

$$
Z_{\mu}\left(H^{+} W^{-\mu}-H^{-} W^{+\mu}\right)
$$

\footnotetext{
${ }^{16}$ We stress that this breaking would be due to the strong sector. This is because $w^{a}=\operatorname{Im}\left(\Upsilon_{L}\right)$ is odd under complex conjugation, so that $w_{3}$ is even under the combined action of complex conjugation and $C_{1}$, which is precisely $C P$ on the spurion - see eqs. 42 and $(59)$.
} 
Of course all these terms require explicit breakdown of $C_{2}$ and a relevant question concerns the possibility to generate them in the $\mathrm{SO}(6) / \mathrm{SO}(4) \times \mathrm{SO}(2)$ models. We have explicitly checked that only $\mathcal{O}_{5}$ is generated in these models, its avatar being, using the notation of section 3 .

$$
\frac{g^{\prime 2}}{16 \pi^{2}} \frac{m_{\rho}^{2}}{g_{\rho}^{2}}\left(\Gamma_{g^{\prime}}^{+}\right)^{\alpha i \beta j} \epsilon^{\alpha \gamma}\left(\mathcal{D}_{\mu}\right)^{\gamma i}\left(\mathcal{D}^{\mu}\right)^{\beta j} .
$$

Notice that only the $T_{3}^{R}$ part of $g^{\prime}$ contributes.

\subsection{Phenomenology of $\mathrm{SO}(6) / \mathrm{SO}(4) \times \mathrm{SO}(2)$ Models}

We here focus on the explicit examples described in section 3 corresponding to the $\mathrm{SO}(6) / \mathrm{SO}(4) \times \mathrm{SO}(2)$ coset. In that case we have

$$
c_{1}=-\frac{1}{2}, \quad c_{2}=0, \quad c_{3}=-\frac{1}{2}, \quad c_{4}=0, c_{5}=0,
$$

where $c_{2}$ and $c_{5}$ are zero at leading order due to the accidental $C_{2}$ symmetry of the coset, and $c_{4}$ vanishes because of the absence, at leading order, of an operator involving the $\mathrm{SO}(4)$ Levi-Civita tensor. From eq. (118) we find that in these models only the first two sum rules of eq. (93) are modified, with the ratios of eq. 97 not being altered. Therefore a precise determination of the coupling of the $C P$-even Higgses, $h_{1}^{0}$ and $h_{2}^{0}$, to $V^{a} V^{a}$ or $V^{a} H^{a}$ will be needed to study the two first sum rules of eq. 933), and possibly unravel the composite nature of the Higgs bosons. Alternatively, one could measure the growth with energy of the $V^{a} V^{a}$ scattering amplitude, that proceeds as in eqs. (4.14)-(4.16) of ref. [5] with $c_{H}=-2 c_{1}=1$. The Higgs couplings to fermions are modified by corrections of order $v^{2} / f^{2}$ arising from eq. 100. The coefficients $c_{t}^{(i)}$ depend on the specific representations of the operator to which the SM fermions couple and are model dependent. For the models of section 3.1 .2 and 3.1 .3 we find respectively

$$
c_{t}^{(1)}=-\frac{1}{2}, \quad c_{t}^{(2)}=c_{t}^{(3)}=\frac{i}{4} \tan \theta, \quad c_{t}^{(4)}=0,
$$

and

$$
c_{t}^{(1)}=-\frac{1}{2}, \quad c_{t}^{(2)}=c_{t}^{(3)}=c_{t}^{(4)}=0
$$

where $c_{t}^{(2)}=c_{t}^{(3)}$ due to the $\mathrm{SO}(4)$ symmetry of the coset. Again, notice that we need to measure the coupling of both $C P$-even Higgs to fermions in order to establish deviations from the sum rules of eq. (100).

Besides the above features, the phenomenology of the explicit models discussed in section 3 will be very similar to that of an elementary $2 \mathrm{HDM}$. The main characteristic will be the approximate custodial symmetry of the Higgs potential and the smallness of $\theta_{h}$, as we explore in the following examples.

\subsubsection{Composite Inert Higgs}

The $C_{2}$-invariant model of section 3.1 .2 corresponds to an inert Higgs scenario, whose phenomenology has been extensively studied in the literature starting with [4]. We can identify $H_{1}$ and $H_{2}$, defined in the basis 
eq. (101), respectively with $H_{\widehat{1}}$ and $H_{\widehat{2}}$ defined in section 3.1.2. As described there, the lightest Higgs, now called $h^{0}$, is $C_{2}$-even and behaves as the SM Higgs with a mass ranging from 150 to $250 \mathrm{GeV}$ for $N=10$ to $N=3$. The other Higgs bosons, $H^{0}, A^{0}$ and $H^{ \pm}$, are $C_{2}$-odd and their masses fulfill the approximate relations

$$
m_{H^{+}}^{2} \simeq m_{A^{0}}^{2}, \quad m_{H^{0}}^{2} \simeq\left(1-\frac{\xi}{3}\right) m_{A^{0}}^{2}
$$

The lightest $C_{2}$-odd Higgs is the neutral $H^{0}$ that is stable with a mass that can be as low as $m_{H^{0}} \sim 500 \mathrm{GeV}$ by taking $N \simeq 10$. The $C_{2}$-odd Higgses can only be pair produced at the LHC through the processes $q q^{\prime} \rightarrow \gamma, Z^{*}, W^{ \pm *} \rightarrow H^{a} H^{b}$, with cross-sections below fb for masses $\sim 500 \mathrm{GeV}$. The strong degeneracy of $H^{+}$and $A^{0}$ implies that the decay channel $H^{+} \rightarrow W^{+} A^{0}$, or vice versa, $A^{0} \rightarrow W^{+} H^{-}$, cannot proceed, and the main decay channels are $H^{+} \rightarrow W^{+} H^{0}$ and $A^{0} \rightarrow Z H^{0}$ where $H^{0}$ being stable escapes from the detector giving missing energy.

The $C_{2}$ symmetry could explicitly be broken, as discussed in the last model of section 3.1.2. In this case, however, we saw that approximate accidental $C_{1} P$ and $C_{2}$ (and custodial) symmetry of the scalar potential force the VEV of $H_{\widehat{2}}$ to be small (see eq. (72) and (73)). We can then still approximately identify $H_{\widehat{1}}$ and $H_{\widehat{2}}$ respectively with $H_{1}$ and $H_{2}$. We also found that the breaking of $C_{1} P \cdot C_{2}$ is further suppressed with respect to that of $C_{2}$ in the scalar potential. Notice that $C_{1} P \cdot C_{2}$ acts as a $C P$ symmetry under which $A^{0}$ is $C P$-even and $H^{0}$ is $C P$-odd. The main consequence of the explicit $C_{2}$ breaking is that now $\mathrm{H}_{2}$ couples to fermions according to eq. 75 , implying that $H^{0}, A^{0}$ and $H^{+}$can be single produced by gluon fusion, decaying mainly into tops and bottoms. Their decay into gauge bosons is suppressed by the small VEV of $H_{2}$, and we estimate that the corresponding branching ratios are always smaller than $1 \%$.

\subsubsection{Almost Inert Higgs}

In the $C_{1} P$-invariant model of section 3.1 .3 , the $C_{2}$ symmetry is preserved by the top Yukawa coupling, but violated by the coupling to the bottom and the tau, since $\tilde{\theta}$ defined in eq. (77) is a free parameter. Furthermore, $C_{2}$ is accidentally preserved in the Higgs potential at order $y_{L}^{2}$. To go from the Higgs doublet basis $\left(H_{\widehat{1}}, H_{\widehat{2}}\right)$ of eq. (63), to the basis $\left(H_{1}, H_{2}\right)$ of eq. (101), where only one Higgs doublet gets a VEV, we must perform a rotation of order $m_{12}^{2} / m_{22}^{2} \sim y_{L}^{2} / g_{\rho}^{2}$ that generates a contribution to $a_{t}$ of this order. Therefore we find a $2 \mathrm{HDM}$ with the following properties:

1) $a_{t} \sim \frac{y_{L}^{2}}{g_{\rho}^{2}}, \quad a_{b, \tau}=\tan \tilde{\theta}_{d, l}$, where $a_{b, \tau}$ is the equivalent of $a_{t}$ for the bottom quark and the tau lepton.

2) $\theta_{h} \sim \frac{y_{L}^{2}}{g_{\rho}^{2}} \xi \sim a_{t} \xi \ll 1$, implying that $h^{0}, H^{0}$ are approximately mass-eigenstates.

3) $m_{h^{0}}^{2} \sim \xi m_{H^{0}}^{2} \sim \frac{N_{c} y_{L}^{2} g_{\rho}^{2} v^{2}}{16 \pi^{2}}$.

4) $m_{H^{+}}^{2} \simeq m_{A^{0}}^{2}, m_{A^{0}}^{2} \simeq m_{H^{0}}^{2}-\frac{1}{3}\left(m_{h^{0}}^{2}+\xi m_{H^{0}}^{2}\right)$. 


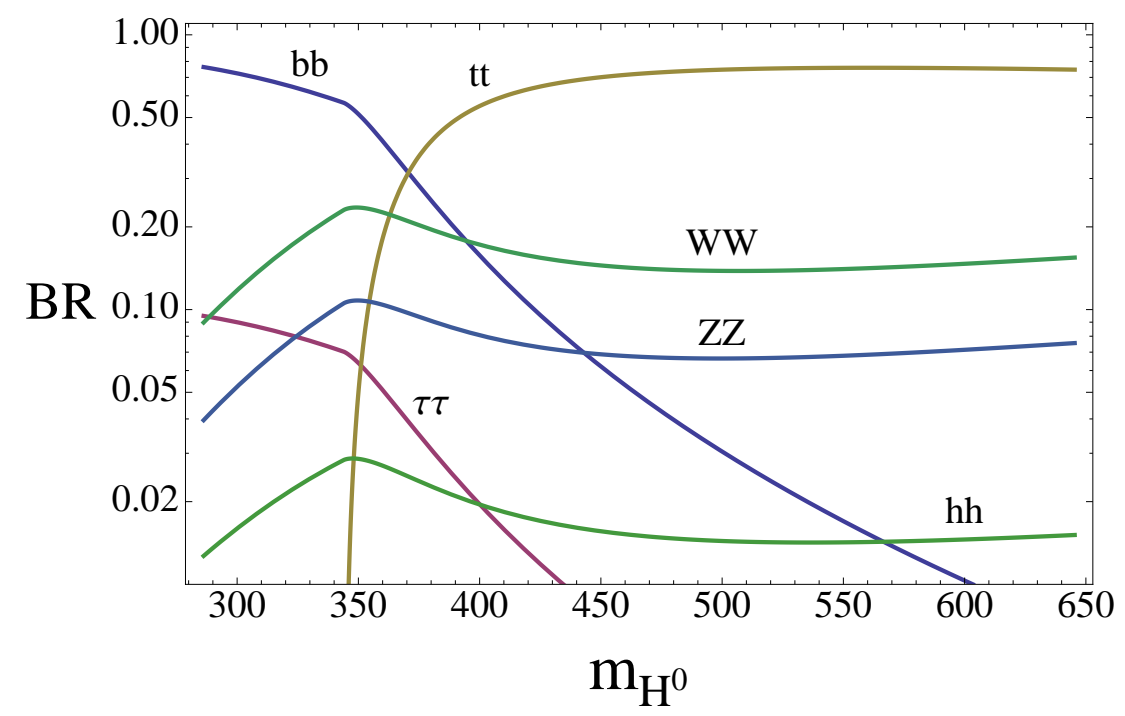

Figure 4: Branching ratios for $H^{0}$ in the almost inert Higgs model.

5) $\lambda_{H^{0} h^{0} h^{0}} \sim \frac{y_{L}^{2}}{g_{\rho}^{2}} \frac{m_{H^{0}}^{2} v}{f^{2}} \sim a_{t} \frac{m_{H^{0}}^{2} v}{f^{2}}$, where $\lambda_{H^{0} h^{0} h^{0}}$ is the Higgs trilinear coupling in the potential.

From the above we can calculate the production cross-sections and branching ratios for the Higgs bosons. We focus on the values $N \sim 8, \xi \sim 0.25$ and $\tan \tilde{\theta}_{d, l} \sim 1$, taking $y_{L}$ ranging from $Y_{t} \sim 1$ to $\sqrt{Y_{t} g_{\rho}} \sim 2.1$. For these values of the parameters, the lightest Higgs $h^{0}$, that behaves as a SM Higgs, has a mass ranging from 150 to $300 \mathrm{GeV}$, while $m_{H^{0}}$ ranges from 300 to $650 \mathrm{GeV}$. The Higgs doublet $H_{2}$ couples to the top with a coupling proportional to $Y_{t} a_{t}$ that takes a value between 0.05 and 0.2 . This implies that single Higgs production for $H^{0}, A^{0}$ and $H^{+}$via gluon fusion is suppressed by $Y_{t}^{2} a_{t}^{2}$ with respect to that of the SM Higgs. Vector boson fusion for $H^{0}$ is also very small since it is suppressed by $\theta_{h}^{2}$. The cross-section for the double-production $\mathrm{H}^{+} \mathrm{H}^{0}$ is of few fb for Higgs masses around $300 \mathrm{GeV}$. The decay channels of the Higgs bosons depend strongly on their masses. We show in Fig. 4 the branching ratio for $H^{0}$ as a function of its mass. Notice that for low mass values $H^{0}$ decays mainly into bottoms, but as its mass increases the channel into gauge bosons becomes sizable. The decay to tops dominates whenever it is kinematically allowed. It is important to remark that the fact that the mass splitting between $H^{0}$ and $A^{0}, H^{+}$goes as $\xi m_{H^{0}}$ implies that the decay channels $H^{0} \rightarrow W H^{+}, Z A^{0}$ are only open for large values of the $H^{0}$ mass. For the mass values given in Fig. 4 these decay channels are always close, but we must emphasize that this is very sensitive to the value of $\xi$ and $m_{h}^{2}$. In Fig. 5 we also show the branching ratios of $H^{+}$and $A^{0}$. It is worth mentioning the branching ratio for $H^{+} \rightarrow W Z / \gamma$ that in this model can reach values $\sim 0.01$, much larger than in a renormalizable $2 \mathrm{HDM}$ where it is induced by top loops and takes a value $\sim 10^{-4}$ for $m_{H^{+}} \sim 300 \mathrm{GeV}$ [15]. In our model this decay width arises mainly from the operator $(112)$ that gives

$$
\Gamma\left(H^{ \pm} \rightarrow W^{ \pm} Z / \gamma\right) \sim \frac{g^{\prime 4}}{8 \pi} \frac{v^{2} m_{H^{+}}^{3}}{m_{\rho}^{4}}
$$



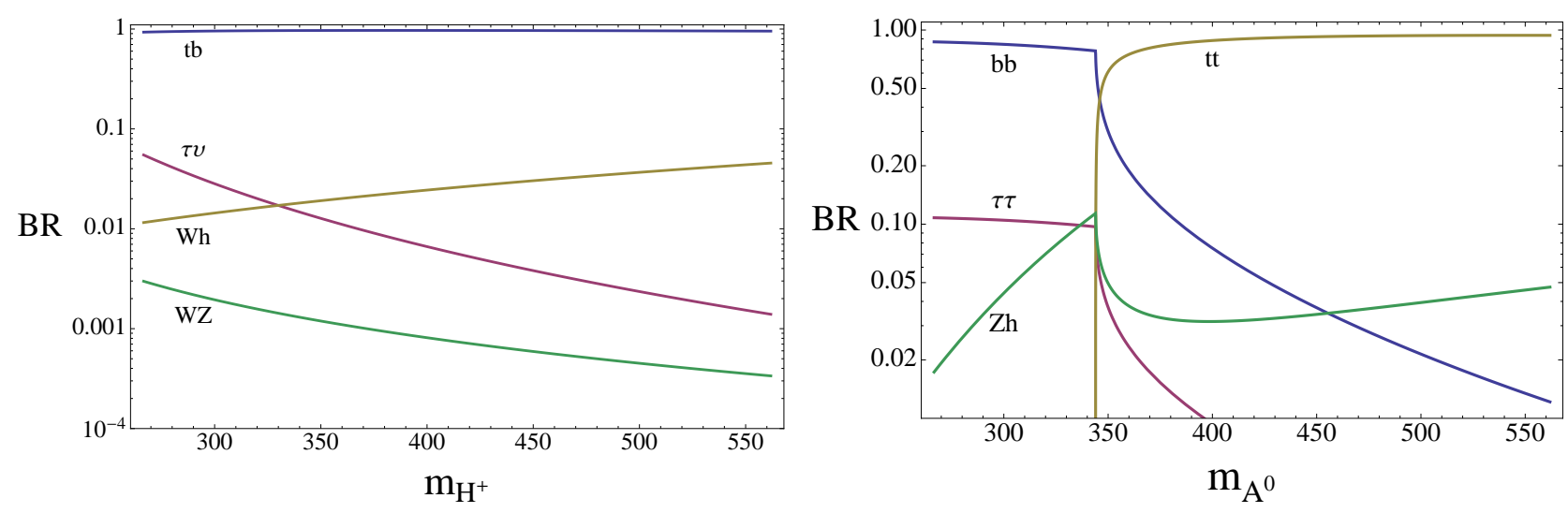

Figure 5: Branching ratios for $H^{+}$and $A^{0}$ in the almost inert Higgs model.

As we increase $N$ and $g_{\rho}$ becomes smaller, we have that $a_{t}$ and $\theta_{h}$ increase. This makes the Higgs easier to be detected. For example, for $N \sim 10$ we can have $h^{0}$ and $H^{0}$ with masses around 170 and $350 \mathrm{GeV}$ respectively, decaying both mainly into gauge bosons.

\section{Summary}

In this paper we have considered the construction and the broad phenomenology of models with a composite Higgs sector featuring two light scalar doublets. The possibility that the Higgs dynamics is determined by light PNGB's from some strong sector is a plausible one. The minimal model, based on $\mathrm{SO}(5) / \mathrm{SO}(4)$, has indeed been extensively studied in recent years. However, there seems to be no a priori, theoretical or experimental, reason to avoid considering less minimal options. Mapping out the structure and phenomenology of non-minimal options is thus a potentially useful thing to do. Our study represents one step in that direction.

Our construction is largely based on the use of symmetries. It is a fact that additional symmetries are typically needed to meet experimental constraints in extensions of the SM. In particular, already in the simple renormalizable 2HDM, in order to control FCNC, either an additional discrete symmetry or minimal flavor violation (that is flavor $\mathrm{SU}(3)^{5}$ selection rules under specific assumptions on the sources of breaking) are invoked. That gives rise to respectively the type I-II and type III models. On the other hand, in composite Higgs models and in technicolor, an approximate $\mathrm{SO}(4)$ symmetry of the strong sector must be assumed in order to control the corrections to $\widehat{T}$. Our study shows that in order to have a phenomenologically acceptable composite $2 \mathrm{HDM}$ it is enough to postulate the strong sector is invariant under $\mathrm{SO}(4)$ times a discrete symmetry. Two possibilities are given for the latter, either $C_{2}$, that is a $Z_{2}$ symmetry distinguishing the two doublets, or $C P$, that we call $C_{1} P$ given its specific action on the strong sector. These discrete symmetries are, maybe surprisingly, essential to control $\widehat{T}$, while Higgs-mediated FCNC can be tamed by the non-linear symmetry $G$ of the strong sector combined with either $C_{2}$ or flavor minimality assumptions similar but different from MFV. Models based on $\mathrm{SO}(6) / \mathrm{SO}(4) \times \mathrm{SO}(2)$ and 
$\mathrm{SU}(5) / \mathrm{SU}(4) \times \mathrm{U}(1)$ can realize our scenario, but we have studied in detail only explicit realizations of the first possibility.

In the spirit of ref. [5] we have only constructed a low-energy effective description of our models. We see no obstacle to "UV completing" our models into a warped compactification, but we do not want to be tied to that perspective. The underlying hypothesis of our scenario is that it UV completes into a 4D CFT possessing the right set of operators, most notably fermions with scaling dimension $d \sim 5 / 2$, in order to implement the partial-compositeness paradigm. Our construction is based on two assumptions. The first one is that the strong sector is broadly characterized by a mass scale $m_{\rho}$ and a coupling strength $g_{\rho}$. The second assumption is that the global symmetry $G$ is only broken by the (linear) coupling of SM fields to strong sector operators. While the coupling to vector bosons is fully fixed by gauge invariance, more freedom exists in the fermionic sector. To fully specify the model we must choose the quantum numbers of the operators that mix with the SM fermions, in particular to the top quark. In this paper we have focussed on two possibilities. In the first class of models, that we indicate by $\{\boldsymbol{6}, \boldsymbol{6}\}$, both left- and right-handed fermions couple to operators in the $\mathbf{6}$ of $\mathrm{SO}(6)$. In the second class, indicated by $\left\{\mathbf{2 0}^{\prime}, \mathbf{1}\right\}$, the left and right-handed fermions couple to respectively a $\mathbf{2 0}^{\prime}$ and to a total singlet.

Under the above assumptions, our methodology to derive the low energy effective Lagrangian relies on the CCWZ formalism and makes broad use of all spurionic symmetries. We find it easier in the CCWZ language, compared to the approach based on the linear Higgs field $\Sigma$ that breaks $G \rightarrow H$, to count and classify the independent invariants at any given order. Our effective Lagrangian is organized as an expansion in derivatives and in powers of the $G$ breaking spurions, the most relevant ones being the top quark proto-Yukwas $y_{L}$ and $y_{R}$, and the gauge couplings $g$ and $g^{\prime}$. One crucial result of our analysis is the emergence of accidental symmetries at the lowest orders in the expansion. On one hand, these accidental symmetries can help the models to meet the phenomenological constraints. On the other hand they provide smoking guns for the whole scenario.

One accidental symmetry that emerges in our analysis up to the order relevant for all experimental tests is the $Z_{2}$ parity in $\mathrm{O}(4)$, defined by $\mathrm{O}(4)=\mathrm{SO}(4) \times P_{L R}$. This symmetry crucially protects the $Z \bar{b} b$ vertex form receiving large corrections. This happy accident, as the analysis of section 2.4 shows, depends on the choice of the strong sector's global group and on the representation to which the left-handed fermions mix. In particular this happens in the MCHM with fermions in the $\mathbf{5}$ and in the composite 2HDM we consider in this paper. The fact that this symmetry is simply accidental, and needs not to be respected by the fundamental strong sector's dynamics, was not appreciated before. Thanks to $P_{L R}$ a larger value of $y_{L}$ and compatibly a larger value of $m_{h}$ can be achieved, without a stark contradiction with EWPT.

More specifically to our $\mathrm{SO}(6) / \mathrm{SO}(4) \times \mathrm{SO}(2)$ models, we also find that $C_{2}, C_{1} P$ and $\mathrm{SO}(3)$ can arise as accidental symmetries of subsectors in the low-energy effective action or simply at lowest order. In particular the leading $O\left(y^{2}\right)$ contribution to the scalar potential is invariant under $C_{2} \times C_{1} P \times \mathrm{SO}(3)$ under the weak assumptions that the strong sector and the top proto-Yukawa respects either $C_{2}$ or $C_{1} P$. 
Indeed in the $\{\mathbf{6}, \mathbf{6}\}$ model, this result holds even when $C_{2}$ is maximally broken by the top proto-Yukawa. Then around the generic vacuum of this model, the Higgs bosons self-interactions and their coupling to vector bosons, respects $C_{2}$ to a good approximation, implying that one of the Higgs doublets, say $H_{2}$, is quasi-inert. However the interactions of both $H_{1}$ and $H_{2}$ to the top maximally break $C_{2}$. The resulting signal is that $A, H^{ \pm}, H$ can be singly produced in gluon fusion and decay dominanty to $t, b$ quarks, with only a tiny branching ratio to vector bosons and to $h$. In the case that $C_{2}$ is completely preserved, we obtain a composite inert Higgs model. In the $\left\{\mathbf{2 0}^{\prime}, \mathbf{1}\right\}$ model, when $C_{1} P$ is a symmetry of the strong sector and of the proto-Yukawas, one has that $C_{2}$ arises as an accidental symmetry of the lowest-order effective action. In this case the second Higgs genuinely behaves like a quasi-inert doublet, including its couplings to fermions. It is therefore mostly doubly produced, even though the underlying strong dynamics maximally breaks $C_{2}$. In that case to reveal the accident and learn about the structure of the theory one would need to observe $C_{2}$ violation in the production of heavy resonances, and not just the PNGBs.

The presence of $\mathrm{SO}(3)$ symmetry at leading order in the Higgs potential can be easily understood by noticing that $y_{L}^{2}$ can be decomposed into a triplet plus a singlet under $\mathrm{SO}(3)$. Given that $h, H$ are singlets and $A, H^{ \pm}$form a triplet, $H^{a}$, and given that the neutrals $h, H, A$ cannot mix by $C_{2}$ and $C_{1} P$, one readily realizes no mass term involving the triplet in $y_{L}^{2}$ can be written. The degeneracy $m_{A} \simeq m_{H^{ \pm}}$is thus one indirect but robust prediction of the composite 2HDM. This is only broken by small effects of order $g^{2}$ and $y_{L}^{4}$. On top of $\mathrm{SO}(3)$ invariance, in the specific models we considered there are additional predictions for the Higgs mass spectrum, arising from the limited number of independent structures at leading order. For instance, in the $\left\{\mathbf{2 0}^{\prime}, \mathbf{1}\right\}$ model the leading $O\left(y^{2}\right)$ potential is determined by three unknown coefficients associated to the three invariants one can write. There is thus one relation among the four parameters $\xi, m_{h}, m_{H}, m_{A}$, given by eq. 81). Notice that one always has $m_{H}>m_{A}$. In the $\{\mathbf{6}, \mathbf{6}\}$ model there are only two parameters at leading order, so that one has in principle an additional prediction at leading $O\left(y^{2}\right)$. However, that just amounts to $m_{h}^{2} \ll m_{H}^{2} / \xi$ corresponding to the fact that in order to have electroweak symmetry breaking it is necessary to tune $m_{h}^{2}$ to be $O\left(y^{4}\right)$ rather than $O\left(y^{2}\right)$. The other prediction is given by eq. (68). Notice that in contrast to the $\left\{\mathbf{2 0}^{\prime}, \mathbf{1}\right\}$ model, here we always have $m_{H}<m_{A}$.

Aside of the above restricted structure which is mostly a consequence of model building constraints, the genuine predictions of a composite $2 \mathrm{HDM}$ model reside in $O(\xi)$ deviation in the Higgs couplings with respect to the elementary case, in the growth with energy of scattering amplitudes involving scalars and longitudinally polarized vectors, and eventually in the production of strongly coupled resonances. Using our effective Lagrangian we have given a general parametrization of the first two classes of effects. Our results generalize the composite Lagrangian of ref. [5]. Putting those effects in evidence at the LHC will be difficult unless $\xi$ is somewhat large, likely above $\sim 0.2$. In the composite 2 HDM the situation does not seem easier. Indeed given the rich set of relations and sum rules implied in the renormalizable 2HDM (see section 4.1), one may have naively expected more dramatic effects when turning on non-renormalizable couplings. In particular $g_{H^{+} W^{-} Z}$, which vanishes at tree level in the renormalizable case, was a candidate 
to potentially large effects. However it turns out that in any realistic composite 2HDM this coupling is also suppressed because of symmetry reasons, as we have discussed in detail. Therefore one does not get dramatic enhancements of the branching ratio for $H^{ \pm} \rightarrow W Z$. Nevertheless it is worth noticing that in the $\left\{\mathbf{2 0}^{\prime}, \mathbf{1}\right\}$ model the branching ratios for $H^{ \pm} \rightarrow W Z / \gamma$ are significantly enhanced over the renormalizable case, basically due to the accidental $C_{2}$ symmetry in the coupling with fermions. That symmetry is instead broken at $O\left(g^{\prime 2}\right)$ in the coupling to vectors.

The conclusion of our study is that realistic composite $2 \mathrm{HDM}$ can reasonably be constructed, showing a procedure that can be used for other composite models with richer Higgs structure. The main signatures that can be extracted at the LHC concern the structure of the spectrum, mainly its peculiar $\mathrm{SO}(3)$ invariance, that can be used to distinguish them from supersymmetric models. Beyond that, we have outlined a rich pattern of deviations from the renormalizable 2HDM. The study of those effects belongs to the worthy motivations of a Linear Collider.

\section{Acknowledgments}

We would like to thank R.Contino and G.Panico for discussions. The work of JM, RR and AW has been partially supported by the Swiss National Science Foundation under contracts 200021-125237 and 200021-116372. The work of AP and JS has been partly supported by CICYT-FEDER-FPA2008-01430, 2009SGR894, AP2006-03102, and ICREA Academia program. 


\section{A Two Higgs SO(4)-invariant derivative interactions}

We want to classify all the dimension-six $\mathrm{SO}(4)$-invariant operators with two derivatives, which are of three different kinds:

1. Operators with $\square$ of the form

$$
\left(\Phi_{i}^{\alpha} \square \Phi_{i}^{\beta}\right)\left(\Phi_{i}^{\gamma} \Phi_{i}^{\delta}\right)
$$

with $\alpha=\widehat{1}, \widehat{2}$ and analogously for $\beta, \gamma$ and $\delta$. There are 12 of them.

2. Operators in which an $\mathrm{SO}(4)$ singlet is formed by contracting one $\Phi^{\alpha}$ field with one derivative $\partial_{\mu} \Phi^{\alpha}$. Those can be written in terms of four objects

$$
\begin{aligned}
d_{\mu}^{\widehat{1}} & \equiv \frac{1}{2} \partial_{\mu}\left(\Phi^{\widehat{1}}\right)^{2}, \\
d_{\mu}^{\widehat{2}} & \equiv \frac{1}{2} \partial_{\mu}\left(\Phi^{\widehat{2}}\right)^{2}, \\
d_{\mu}^{\widehat{1} \hat{2}} & \equiv \frac{1}{2} \partial_{\mu}\left(\Phi^{\widehat{1}} \cdot \Phi^{\widehat{2}}\right), \\
c_{\mu} & \equiv \Phi^{\widehat{1}} \partial_{\mu} \Phi^{\widehat{2}}-\Phi^{\widehat{2}} \partial_{\mu} \Phi^{\widehat{1}},
\end{aligned}
$$

by forming all possible Lorentz vector products. However, since $\partial^{\mu} c_{\mu}=0$, the only non-vanishing contraction of $c_{\mu}$ is with itself, so that we have $1+6=7$ independent invariants in this class. Operators in which an $\mathrm{SO}(4)$ singlet is formed by contracting two derivatives $\partial_{\mu} \Phi^{\alpha}$ can obviously be rewritten as the previous ones by integration by parts.

3. The $P_{L R}$-odd operator

$$
\frac{c_{\epsilon}}{f^{2}} \epsilon^{i j k l} \Phi_{i}^{\hat{1}} \Phi_{j}^{\hat{2}} D_{\mu} \Phi_{k}^{\hat{1}} D_{\mu} \Phi_{l}^{\hat{2}} .
$$

All the 12 operators of the first class can be eliminated by the 12 field redefinitions of the form

$$
\Phi_{i}^{\alpha} \rightarrow \Phi_{i}^{\alpha}+A \Phi_{i}^{\beta}\left(\Phi^{\gamma} \cdot \Phi^{\delta}\right)
$$

so that we are left with the following operators

$$
\begin{aligned}
& \mathcal{L}_{6 d}=c_{H_{1}}\left(d^{\widehat{1}}\right)_{\mu}\left(d^{\widehat{1}}\right)^{\mu}+c_{H_{2}}\left(d^{\widehat{2}}\right)_{\mu}\left(d^{\widehat{2}}\right)^{\mu}+c_{H_{12}}\left(d^{\widehat{1} \hat{2}}\right)_{\mu}\left(d^{\widehat{1} \widehat{2}}\right)^{\mu}+c_{H_{1} H_{2}}\left(d^{\widehat{1}}\right)_{\mu}\left(d^{\widehat{2}}\right)^{\mu} \\
& +c_{H_{1} H_{12}}\left(d^{\widehat{1}}\right)_{\mu}\left(d^{\widehat{1} \widehat{\underline{2}}}\right)^{\mu}+c_{H_{2} H_{12}}\left(d^{\widehat{2}}\right)_{\mu}\left(d^{\widehat{1} \widehat{\underline{2}}}\right)^{\mu}+c_{T} c_{\mu} c^{\mu}+c_{\epsilon} \epsilon^{i j k l} \Phi_{i}^{\hat{1}} \Phi_{j}^{\hat{2}} D_{\mu} \Phi_{k}^{\hat{1}} D_{\mu} \Phi_{l}^{\widehat{2}} .
\end{aligned}
$$

As also explained in the text, it might seem that one of the field redefinition in eq. (126) (in particular, $\left.\Phi^{\hat{1}} \rightarrow \Phi^{\widehat{2}}+A \Phi^{\widehat{1}} \Phi^{\hat{1}} \cdot \Phi^{\hat{1}}\right)$ cannot be performed if willing to remain in a basis where $\Phi^{\widehat{2}}$ does not take $\mathrm{VEV}$. The VEV of $\Phi^{\hat{2}}$ induced by this field redefinition, however, can always be eliminated by performing a further redefinition, which consists in an $\mathrm{SO}(2)$ rotation in the $\left(\Phi^{\widehat{1}}, \Phi^{\widehat{2}}\right)$ plane. By this second rotation the operators in eq. (127) merely rotate into each other.

Finally for the $\mathrm{SO}(6) / \mathrm{SO}(4) \times \mathrm{SO}(2)$ coset studied above the coefficients are given by

$$
c_{H_{1}}=\frac{1}{2}, c_{H_{2}}=\frac{1}{2}, c_{H_{12}}=1, c_{H_{1} H_{2}}=0, c_{H_{1} H_{12}}=0, c_{H_{1} H_{12}}=0, c_{T}=-\frac{1}{4}, c_{\epsilon}=0 .
$$




\section{References}

[1] D. B. Kaplan and H. Georgi, Phys. Lett. B 136 (1984) 183.

[2] K. Agashe, R. Contino and A. Pomarol, Nucl. Phys. B 719 (2005) 165 arXiv:hep-ph/0412089.

[3] S. R. Coleman, J. Wess and B. Zumino, Phys. Rev. 177 (1969) 2239; C. G. . Callan, S. R. Coleman, J. Wess and B. Zumino, Phys. Rev. 177 (1969) 2247.

[4] R. Barbieri, L. J. Hall, V. S. Rychkov, Phys. Rev. D74, 015007 (2006). hep-ph/0603188.

[5] G. F. Giudice, C. Grojean, A. Pomarol and R. Rattazzi, JHEP 0706 (2007) 045 arXiv:hepph/0703164.

[6] R. Contino, L. Da Rold and A. Pomarol, Phys. Rev. D 75 (2007) 055014 arXiv:hep-ph/0612048.

[7] D. B. Kaplan, Nucl. Phys. B 365 (1991) 259.

[8] R. Contino, T. Kramer, M. Son and R. Sundrum, JHEP 0705 (2007) 074 arXiv:hep-ph/0612180.

[9] B. Gripaios, A. Pomarol, F. Riva et al., JHEP 0904, 070 (2009). arXiv:0902.1483 [hep-ph]]

[10] G. D'Ambrosio, G. F. Giudice, G. Isidori, A. Strumia, Nucl. Phys. B645, 155-187 (2002). hepph/0207036.

[11] K. Agashe, R. Contino, Phys. Rev. D80, 075016 (2009). arXiv:0906.1542 [hep-ph]].

[12] C. Csaki, A. Falkowski, A. Weiler, JHEP 0809, 008 (2008). arXiv:0804.1954 [hep-ph]].

[13] G. Cacciapaglia, C. Csaki, J. Galloway, G. Marandella, J. Terning and A. Weiler, JHEP 0804, 006 (2008) arXiv:0709.1714 [hep-ph]].

[14] K. Agashe, R. Contino, L. Da Rold, A. Pomarol, Phys. Lett. B641, $62-66$ (2006). hep-ph/0605341.

[15] A. Mendez, A. Pomarol, Nucl. Phys. B349 (1991) 369-380; M. Capdequi Peyranere, H. E. Haber, P. Irulegui, Phys. Rev. D44 (1991) 191-201. 